\title{
SEMICLASSICAL ORTHOGONAL POLYNOMIAL SYSTEMS ON NONUNIFORM LATTICES, DEFORMATIONS OF THE ASKEY TABLE, AND ANALOGUES OF ISOMONODROMY
}

\author{
N. S. WITTE
}

\begin{abstract}
A $\mathbb{D}$-semiclassical weight is one which satisfies a particular linear, first-order homogeneous equation in a divided-difference operator $\mathbb{D}$. It is known that the system of polynomials, orthogonal with respect to this weight, and the associated functions satisfy a linear, first-order homogeneous matrix equation in the divided-difference operator termed the spectral equation. Attached to the spectral equation is a structure which constitutes a number of relations such as those arising from compatibility with the three-term recurrence relation. Here this structure is elucidated in the general case of quadratic lattices. The simplest examples of the $\mathbb{D}$-semiclassical orthogonal polynomial systems are precisely those in the Askey table of hypergeometric and basic hypergeometric orthogonal polynomials. However within the $\mathbb{D}$-semiclassical class it is entirely natural to define a generalization of the Askey table weights which involve a deformation with respect to new deformation variables. We completely construct the analogous structures arising from such deformations and their relations with the other elements of the theory. As an example we treat the first nontrivial deformation of the Askey-Wilson orthogonal polynomial system defined by the $q$-quadratic divided-difference operator, the Askey-Wilson operator, and derive the coupled first-order divided-difference equations characterizing its evolution in the deformation variable. We show that this system is a member of a sequence of classical solutions to the $E_{7}^{(1)} q$-Painlevé system.
\end{abstract}

\section{Contents}

1. Background and motivation . . . . . . . . . . . . . . . . 128

2. Divided-difference calculus of the quadratic lattices . . . . . . . . . . . . . . 133

3. Orthogonal polynomial systems on the nonuniform lattice . . . . . . . . . . . 138

4. Spectral differences . . . . . . . . . . . . . . . . . . . . . . . 148

5. The $M=2, L=0$ cases and the Askey-Wilson polynomials . . . . . . . . . 166

6. Deformation differences . . . . . . . . . . . . . . . . . . . . . 173

7. The $M=3, L=1$ cases and deformation of the Askey-Wilson OPS . . . . . 191

References . . . . . . . . . . . . . . . . . . . . . . . 228

Received May 9, 2012. Accepted October 31, 2014.

2010 Mathematics Subject Classification. Primary 39A05; Secondary 42C05, 34M55, 34M56, 33C45, 37K35. 


\section{$\S 1$. Background and motivation}

We propose a method for constructing systems of linear divided-difference equations which are analogues of isomonodromic linear differential equations and therefore isomonodromic interpretations or Lax pairs of the known qPainlevé and difference Painlevé equations, such as those in the Sakai scheme (see [86]). What is meant by an analogue of a monodromy matrix turns out to be a connection matrix, appropriate to the class of lattices defining the divided-difference operators under consideration. In essence, our method constructs a particular isomonodromic analogue system from an orthogonal polynomial system, orthogonal with respect to a generalization or deformation of a weight with discrete or countable support on a class of nonuniform quadratic lattices. We then deduce a number of linear divided-difference equations that this system satisfies and show that their pairwise compatibility holds provided the coefficients of the linear system obey evolution equations of the difference or $q$-Painlevé type. This is a very natural extension of the Fokas-Its-Kitaev construction (see [49], [23], [24]) at the heart of Riemann-Hilbert techniques.

Our method is independent of and distinct from other approaches which we briefly recount here. The first studies to construct Lax pairs for the $q$ Painlevé equations were those of Jimbo and Sakai [50] and Sakai [87], [88], using the Birkhoff theory of linear $q$-difference equations $Y(q x)=A(x) Y(x)$ and imposing the condition that the connection matrix was independent of the zeros of $\operatorname{det} A(x)$. However this approach has not been extended beyond the $D_{5}^{(1)}$ or $E_{6}^{(1)}$ cases (the latter case only found from a degeneration of the two-variable extension of the former). Another distinct approach which is founded upon the notion of the $\tau$-function of a rational d-connection is the Arinkin-Borodin theory (see [17], [2], [3]), which has been applied to the difference Painlevé equations. Our approach is similar in spirit to that of Rains [85], who has treated the master elliptic Painlevé equation, in that an explicit construction is made of the solution to the linear problem which contains a multiple integral representation of the orthogonal rational function. However, we will not make direct contact with this theory because it requires us to consider orthogonal rational functions on elliptic lattices, which is a generalization beyond the class of lattices considered here. In addition, we believe that the discrete Riemann-Hilbert approach, as formulated by Borodin in [16] and applied to two examples and extended to further cases in [18], shares many features with the present study, and 
one should be able to construct a Riemann-Hilbert formulation of our own theory. Recently Yamada has constructed Lax pairs for the $q$-Painlevé equations for the systems with symmetries $E_{8}^{(1)}, E_{7}^{(1)}, E_{6}^{(1)}$ by a reduction from the elliptic form of the $E_{8}^{(1)}$ Painlevé equation (see [98], [99]); however, no theoretical construction from first principles was proposed in the individual cases.

The approach we propose here has been successfully employed for the isomonodromic systems and Painlevé equations (see, e.g., [66], [64], [61], [13], [49], [19], [21], [26], [93], [27], [94]), and while most of the findings are reproductions of known ones, they have led to novel results hitherto not found using other treatments, such as the discrete Garnier systems in [95]. An important feature of our approach is that it is strongly motivated by a probabilistic setting, namely, that of the theory of random matrices and more generally determinantal point processes where the classical weights in the Askey table appear in the one-body factors of the eigenvalue probability density functions (see [25]). Some preliminary exploration of the program we propose here has already been initiated but not carried through to its logical conclusion, and we will delay citing this work until the body of our paper where it is directly relevant. However, we should point out that we are most indebted to the pioneering work of Magnus in [60] and [62].

The essential elements of our approach are the following.

(A) The classification of special nonuniform lattices (SNUL) of quadratic type (see [60]), their associated divided-difference operators $\mathbb{D}_{x}$ and $\mathbb{M}_{x}$, and their rules of calculus, which applies to orthogonal or bi-orthogonal polynomial systems. Of significance is the fact that in general these lattices possess two fixed points which we denote $x_{L}, x_{R}$.

(B) The notion of a $\mathbb{D}$-semiclassical weight $w(x)$ which is characterized by an analogue of the Pearson equation (see Definition 4.1; see also [60], [62])

$$
\mathbb{D}_{x} w(x)=\frac{2 V(x)}{W(x)} \mathbb{M}_{x} w(x)
$$

where $W(x), V(x)$ are polynomials in $\mathbb{C}[x]$.

(C) The orthogonal polynomial system (OPS) defined by such a weight on the SNUL $Y_{n}(x) \in \mathbb{C}^{2 \times 2}$ satisfies a three-term recurrence relation, which in 
our context is a particular Schlesinger transformation $n \mapsto n+1$ (see (3.29))

$$
Y_{n+1}(x)=K_{n}(x) Y_{n}(x) .
$$

(D) The semiclassical character implies, under fairly weak conditions, a spectral structure on the "isomonodromic" system $Y_{n}(x)$ (see [60], [62]; see also Proposition 4.4), namely, that it satisfies the linear divided-difference equation

$$
\mathbb{D}_{x} Y_{n}(x)=A_{n}(x) \mathbb{M}_{x} Y_{n}(x)
$$

Here $A_{n}(x)$ is rational in $x$ and the degrees of its numerator and denominator with respect to $x$ are independent of $n$.

(E) Parallel to the spectral structure is a deformation structure, whereby the weight and the system acquires a $u$ dependence, constrained by another Pearson relation (see Definition 6.1)

$$
\mathbb{D}_{u} w(x ; u)=\frac{2 S(x ; u)}{R(x ; u)} \mathbb{M}_{u} w(x ; u),
$$

where $R(x ; u), S(x ; u)$ are polynomials in $\mathbb{C}[x]$.

(F) This also has a direct consequence for the "isomonodromic" system $Y_{n}(x ; u)$, a deformation structure (see Proposition 6.6), and a second associated linear divided-difference equation

$$
\mathbb{D}_{u} Y_{n}(x ; u)=B_{n}(x ; u) \mathbb{M}_{u} Y_{n}(x ; u),
$$

where $B_{n}(x ; u)$ is also rational in $x$.

(G) The compatibility relations implied by this overdetermined "isomonodromic" system $Y_{n}(x ; u)$ then lead to a number of conclusions - the deformation matrix $B_{n}(x ; u)$ is expressible in terms of the spectral matrix $A_{n}(x ; u)$ at neighboring lattice nodes which we refer to as closure (see Proposition 7.8), and furthermore relations exist between components of the spectral matrix at two consecutive nodes on the $u$-lattice which, given a suitable parameterization of this matrix by appropriate coordinates, is a recurrence relation on the deformation lattice. Our key results for such recurrence relations are given in Propositions 7.9, 7.10, 7.11, and 7.12.

(H) In our approach we derive a preservation property for the connection matrix defined as

$$
P(x ; u):=\left(Y_{R}(x ; u)\right)^{-1} Y_{L}(x ; u),
$$


where $Y_{L, R}(x ; u)$ are local fundamental solutions of the spectral equation about $x_{L, R}$. Specifically this implies that

$$
\mathbb{D}_{x} P(x ; u)=0, \quad \mathbb{D}_{u} P(x ; u)=0
$$

as a consequence of our assumptions.

In our approach we can sidestep a number of issues, which are important to be sure, but do not affect the outcome. So we postpone deeper considerations of an analytic or algebraic theory of linear systems of divided-difference operators on these lattices for subsequent studies; however our present work will provide concrete illustrative examples for such an investigation. We are referring to, for example, issues of a Galois theory for $\mathbb{D}$-difference equations, a Birkhoff theory for the local character of the solutions to systems of linear first-order $\mathbb{D}$-difference equations, and analogues of monodromy for $\mathbb{D}$-difference equations.

Our approach poses the question concerning a correspondence between the system of hypergeometric and basic hypergeometric orthogonal polynomial systems generalizing the classical systems, known as the Askey table, as represented in reference work [56], and the most complete system of elliptic, $q$-difference and difference analogues of the Painlevé equations, the Sakai scheme (see [86]). A correspondence between the Sakai scheme and the Askey table would explain the occurrence of many features that have been discovered recently, such as the appearance of basic hypergeometric functions in their classical solutions. We have already referred to the correspondence between the classical OPS Hermite, Laguerre, and Jacobi and the classical solutions to Painlevé IV, V, and VI, respectively. Among the difference and $q$-difference OPS, the current evidence for such a correspondence can be summarized in Table 1.

The layout of this work loosely follows the plan given above.

In Section 2 we describe the classification of the quadratic lattices and their divided-difference calculus, with special emphasis on the example of the master class, the $q$-quadratic lattice. Section 3 is devoted to the reformulation of orthogonal polynomial system theory necessary for systems with a weight having support on a general quadratic lattice, and some analytical results for the $q$-quadratic lattice. The spectral structures are laid out in Section 4 for a general quadratic lattice, starting with the definition of a $\mathbb{D}$-semiclassical weight and developing the consequences of this for the OPS. We also describe the compatibility relations of this structure with the three-term recurrence relations, and this leads to a generalization of the 
Table 1: The Askey table-Sakai scheme correspondence ranked according to the degeneration pathway from the master case. The affine Weyl group refers to the symmetry group of Bäcklund transformations for the integrable system. Only the discrete lattices are included here.

\begin{tabular}{|c|c|c|}
\hline Base OPS & Integrable system & Reference \\
\hline little $q$-Jacobi & $D_{5}^{(1)}$, full $q-\mathrm{P}_{\mathrm{VI}}$ & {$[79]$} \\
\hline Pastro [80] & $D_{5}^{(1)}$, full $q-\mathrm{P}_{\mathrm{VI}}$ & {$[14]$} \\
\hline little $q$-Jacobi & $D_{5}^{(1)}$, special $q-\mathrm{P}_{\mathrm{VI}}$ & {$[18]$} \\
\hline$q$-Krawtchouk & $D_{5}^{(1)}$, special $q-\mathrm{P}_{\mathrm{VI}}$ & {$[18]$} \\
\hline$q$-Charlier & $D_{5}^{(1)}$, degenerate $q-\mathrm{P}_{\mathrm{VI}}$ & {$[18]$} \\
\hline$q$-Freud & $A_{4}^{(1)}$, degenerate $q-\mathrm{P}_{\mathrm{V}}$ & {$[15]$} \\
\hline \hline Meixner, Krawtchouk & $d-\mathrm{P}_{\mathrm{V}}$ & {$[18]$} \\
\hline Charlier & $d-\mathrm{P}_{\mathrm{IV}}$ & {$[18]$} \\
\hline
\end{tabular}

Laguerre-Freud equations. An explicit example of the foregoing theory is the lowest case - and from our point of view, the trivial case - of the q-quadratic lattice which is denoted by the label $M=2$. This is dealt with in Section 5 . We recover the Askey-Wilson OPS and demonstrate that every aspect of this system can be derived in an efficient manner using the theoretical tools developed in the previous section.

In Section 6 we introduce an (or many) auxiliary variable and demand that the weight satisfies an analogous $\mathbb{D}$-semiclassical relation to the spectral relation, on a general lattice not necessarily the same as that for the spectral variable. This constitutes a deformation of the weight which leaves it having the same structure as the original. From this we develop a parallel theoretical analysis of the orthogonal polynomial system with respect to this deformation. We now have two compatibility relations with this deformation structure - one with the three-term recurrence and the other with the spectral structure. For the present purposes, this completes our task for the general theory.

In Section 7 we treat a natural deformation of the Askey-Wilson weight, but not by any means the only one, to the $q$-quadratic lattice. Here we construct parameterizations of the spectral and deformation matrices, the closure relations between the two structures, determine the coordinate transformations to a set of variables, and derive the evolution equations for this 
system (see Propositions 7.9, 7.10, 7.11, and 7.12). Finally, we offer evidence that our system is a classical solution of the $E_{7}^{(1)} q$-Painlevé system.

\section{§2. Divided-difference calculus of the quadratic lattices}

Divided-difference operators associated with the special nonuniform lattices have appeared in many studies of orthogonal polynomials of a discrete variable (see, e.g., the early studies by Hahn [36], [35], [37], [38], the foundational work by Askey and Wilson [4], and the monograph of Nikiforov, Suslov, and Uvarov [78]). The employment of divided-difference operators such as the Askey-Wilson and Wilson operators has been commonplace in studies of the Askey table of hypergeometric orthogonal polynomial systems too numerous to cite here (see the monographs by Ismail [43] and Lesky [59]).

Let $\Pi_{n}[x]$ denote the linear space of polynomials in $x$ over $\mathbb{C}$ with degree at most $n \in \mathbb{Z}_{\geq 0}$. In pioneering investigations, Magnus [60], [62] provided a geometrical understanding of these lattices and their divided-difference operators which we relate here briefly. If we define the divided-difference operator $(\mathrm{DDO}) \mathbb{D}_{x}$ by

$$
\mathbb{D}_{x} f(x)=\frac{f\left(\iota_{+}(x)\right)-f\left(\iota_{-}(x)\right)}{\iota_{+}(x)-\iota_{-}(x)},
$$

then a simple consequence of the condition that $\mathbb{D}_{x}: \Pi_{n}[x] \rightarrow \Pi_{n-1}[x]$ for all $n \in \mathbb{N}$ is that $\iota_{ \pm}(x)$ are the two $y$-roots of the quadratic equation

$$
\mathcal{A} y^{2}+2 \mathcal{B} x y+\mathcal{C} x^{2}+2 \mathcal{D} y+2 \mathcal{E} x+\mathcal{F}=0 .
$$

The functions $\iota_{ \pm}(x)$ satisfy

$$
\begin{aligned}
\iota_{+}(x)+\iota_{-}(x) & =-2 \frac{\mathcal{B} x+\mathcal{D}}{\mathcal{A}}, \\
\iota_{+}(x) \iota_{-}(x) & =\frac{\mathcal{C} x^{2}+2 \mathcal{E} x+\mathcal{F}}{\mathcal{A}},
\end{aligned}
$$

and their inverse functions $\iota_{ \pm}^{-1}$ are defined by $\iota_{ \pm}^{-1}\left(\iota_{ \pm}(x)\right)=x$. For a given $y$ value, the quadratic (2.2) defines two $x$-roots if $\mathcal{C} \neq 0$, which are consecutive points on the $x$-lattice, $x_{s}:=x(s), x_{s+1}:=x(s+1)$ parameterized by the variable $s$, and therefore defines a map $x_{s} \mapsto x_{s+1}$. Conversely, for a given $x$-value the quadratic defines two $y$-roots if $\mathcal{A} \neq 0$, which are consecutive points on a dual lattice, the $y$-lattice, $y_{s}:=y(s)=\iota_{-}(x(s)), y_{s+1}:=y(s+1)=$ 
$\iota_{+}(x(s))$, which generally is distinct from the $x$-lattice. We will employ an operator notation for the mappings from points on the direct lattice to the dual lattice $E_{x}^{ \pm} f(x):=f\left(\iota_{ \pm}(x)\right)$ so that

$$
\mathbb{D}_{x} f(x):=\frac{f\left(\iota_{+}(x)\right)-f\left(\iota_{-}(x)\right)}{\iota_{+}(x)-\iota_{-}(x)}=\frac{E_{x}^{+} f-E_{x}^{-} f}{E_{x}^{+} x-E_{x}^{-} x},
$$

for arbitrary functions $f(x)$. The inverse functions $\iota_{ \pm}^{-1}(x)$ define operators $\left(E^{ \pm}\right)^{-1}$ which map points on the dual lattice to the direct lattice and also an adjoint to the divided-difference operator $\mathbb{D}_{x}$

$$
\mathbb{D}_{x}^{*} f(x):=\frac{f\left(\iota_{+}^{-1}(x)\right)-f\left(\iota_{-}^{-1}(x)\right)}{\iota_{+}^{-1}(x)-\iota_{-}^{-1}(x)}=\frac{\left(E_{x}^{+}\right)^{-1} f-\left(E_{x}^{-}\right)^{-1} f}{\left(E_{x}^{+}\right)^{-1} x-\left(E_{x}^{-}\right)^{-1} x} .
$$

The composite operators $E_{x}:=\left(E_{x}^{-}\right)^{-1} E_{x}^{+}$and $E_{x}^{-1}=\left(E_{x}^{+}\right)^{-1} E_{x}^{-}$map between consecutive points on the direct lattice.

However in the situation of a symmetric quadratic equation $\mathcal{A}=\mathcal{C}$ and $\mathcal{D}=\mathcal{E}$, which entails no loss of generality, then we have $\left(E_{x}^{+}\right)^{-1}=E_{x}^{-}$and $\left(E_{x}^{-}\right)^{-1}=E_{x}^{+}$. Consequently, there is no distinction between the divideddifference operator and its adjoint, and hereafter we adopt this simplification. For example, one useful consequence of this choice is that the ratio

$$
\frac{\left(x-E^{+} u\right)\left(x-E^{-} u\right)}{\left(E^{-} x-u\right)\left(E^{+} x-u\right)}=\frac{\mathcal{A} x^{2}+2 \mathcal{B} x u+\mathcal{C} u^{2}+2 \mathcal{D} x+2 \mathcal{E} u+\mathcal{F}}{\mathcal{A} u^{2}+2 \mathcal{B} u x+\mathcal{C} x^{2}+2 \mathcal{D} u+2 \mathcal{E} x+\mathcal{F}}=1
$$

for all $x, u$. A companion operator to the divided-difference operator $\mathbb{D}_{x}$ is the mean or average operator $\mathbb{M}_{x}$ defined by

$$
\mathbb{M}_{x} f(x)=\frac{1}{2}\left[f\left(\iota_{+}(x)\right)+f\left(\iota_{-}(x)\right)\right]
$$

so that the property $\mathbb{M}_{x}: \Pi_{n}[x] \rightarrow \Pi_{n}[x]$ is ensured by the condition we imposed upon $\mathbb{D}_{x}$.

Definition 2.1. Henceforth we use the shorthand for the difference in consecutive $y$ points $\Delta y(x):=\iota_{+}(x)-\iota_{-}(x)$. We introduce the notion of fixed points, whereby $\Delta y^{2}\left(x_{F}\right)=0$, or $\iota_{+}\left(x_{F}\right)=\iota_{-}\left(x_{F}\right)$ and are given as the roots of $\left(\mathcal{B}^{2}-\mathcal{A C}\right) x_{F}^{2}+2(\mathcal{B D}-\mathcal{A E}) x_{F}+\mathcal{D}^{2}-\mathcal{A F}=0$.

Assuming $\mathcal{A C} \neq 0$, one can classify these nonuniform quadratic lattices (or SNUL) according to two parameters: the discriminant $\mathcal{B}^{2}-\mathcal{A C}$ and 
Table 2: The nonuniform lattices of quadratic type.

\begin{tabular}{|c|c|c|c|c|c|}
\hline $\mathcal{B}^{2}-\mathcal{A C}$ & $\Theta$ & Conic & Lattice & Canonical DDO & Notes \\
\hline 0 & 0 & parallel lines & linear & forward difference & \\
\hline$>0$ & 0 & intersecting lines & $q$-linear & $q$-difference & \\
\hline 0 & $<0$ & parabola & quadratic & Wilson & \\
\hline$>0$ & $<0$ & hyperbola & $q$-quadratic & Askey-Wilson & $q$ real \\
\hline$<0$ & $<0$ & ellipse & $q$-quadratic & Askey-Wilson & $|q|=1$ \\
\hline
\end{tabular}

$$
\Theta=\operatorname{det}\left(\begin{array}{lll}
\mathcal{A} & \mathcal{B} & \mathcal{D} \\
\mathcal{B} & \mathcal{C} & \mathcal{E} \\
\mathcal{D} & \mathcal{E} & \mathcal{F}
\end{array}\right),
$$

or $\mathcal{A} \Theta=\left(\mathcal{B}^{2}-\mathcal{A C}\right)\left(\mathcal{D}^{2}-\mathcal{A F}\right)-(\mathcal{B D}-\mathcal{A} \mathcal{E})^{2}$. There are four primary classes: the linear lattice, the linear $q$-lattice, the quadratic lattice, and the $q$-quadratic lattice, which are given in Table 2. The $q$-quadratic lattice, in its general nonsymmetrical form, is the most general case and the other lattices can be found from this by limiting processes.

This classification of lattices for polynomial systems can be extended to rational function systems (see [89], [90], [63]), and in this case one has a lattice characterized by a biquadratic relation and parameterized by elliptic functions (see [39]; see also [12, Section 15.10]). However, for the purposes of studying the Askey table, we will not pursue this direction.

For the quadratic class of lattices the parameterization on $s$ can be made explicit through the trigonometric/hyperbolic functions or their degenerations so we can employ a parameterization such that $\iota_{-}(x(s))=y(s)=$ $x(s-1 / 2)$ and $\iota_{+}(x(s))=y(s+1)=x(s+1 / 2)$. We denote the totality of lattice points by $G[x]:=\{x(s): s \in \mathbb{Z}\}$ with the point $x(0)=x$ as the basal point, and of the dual lattice by $\tilde{G}[x]:=\left\{x(s): s \in \mathbb{Z}+\frac{1}{2}\right\}$.

Having established the basic properties of the divided-difference operators, we can deduce key elements of their calculus. A consequence of the general definition of the divided-difference operators are the following identities:

(i) the product or Leibniz formulas

$$
\begin{aligned}
\mathbb{D}_{x} f g & =\mathbb{D}_{x} f \mathbb{M}_{x} g+\mathbb{M}_{x} f \mathbb{D}_{x} g, \\
\mathbb{M}_{x} f g & =\mathbb{M}_{x} f \mathbb{M}_{x} g+\frac{1}{4} \Delta y^{2} \mathbb{D}_{x} f \mathbb{D}_{x} g,
\end{aligned}
$$


(ii) the inverse formulas

$$
\mathbb{M}_{x} \frac{1}{f}=\frac{\mathbb{M}_{x} f}{E_{x}^{+} f E_{x}^{-} f}, \quad \mathbb{D}_{x} \frac{1}{f}=-\frac{\mathbb{D}_{x} f}{E_{x}^{+} f E_{x}^{-} f},
$$

(iii) and the commutativity formulas

$$
\mathbb{M}_{u} \mathbb{M}_{x}=\mathbb{M}_{x} \mathbb{M}_{u}, \quad \mathbb{M}_{u} \mathbb{D}_{x}=\mathbb{D}_{x} \mathbb{M}_{u}, \quad \mathbb{D}_{u} \mathbb{D}_{x}=\mathbb{D}_{x} \mathbb{D}_{u}
$$

The other side of our divided-difference calculus concerns the definition and properties of analogues to integrals. We define the $\mathbb{D}$-Integral of a function defined on the $x$-lattice $f: G[x] \rightarrow \mathbb{C}$ with basal point $x_{0}$ by the Riemann sum over the lattice points

$$
\begin{aligned}
I[f]\left(x_{0}\right) & =\int_{G} \mathbb{D} x f(x) \\
& :=\sum_{s \in \mathbb{Z}}\left(\iota_{+}(s)-\iota_{-}(s)\right) f(x(s)) \\
& =\sum_{s \in \mathbb{Z}} \Delta y\left(x_{s}\right) f\left(x_{s}\right),
\end{aligned}
$$

where the sum is either a finite subset of $\mathbb{Z}$, namely, $\{0, \ldots, \mathfrak{N}\}$, or $\mathbb{Z}_{\geq 0}$, $\mathbb{Z}$. This definition reduces to the usual definition of the difference integral and the Thomae-Jackson $q$-integrals in the canonical forms of the linear and $q$-linear lattices, respectively. A number of properties flow from this definition:

(i) an analogue of the fundamental theorem of calculus

$$
\int_{x_{0} \leq x_{s} \leq x_{\mathfrak{N}}} \mathbb{D} x \mathbb{D}_{x} f(x)=f\left(E_{x}^{+} x_{\mathfrak{N}}\right)-f\left(E_{x}^{-} x_{0}\right),
$$

(ii) an analogue of integration by parts for two functions $f(x), g(x)$

$$
\begin{aligned}
& \int_{x_{0} \leq x_{s} \leq x_{\mathfrak{N}}} \mathbb{D} x f(x) \mathbb{D}_{x} g(x) \\
& =-\int_{x_{0} \leq x_{s} \leq x_{\mathfrak{N}}} \mathbb{D}\left(E_{x}^{+} x\right) \mathbb{D}_{x} f\left(E_{x}^{+} x\right) g\left(E_{x}^{+} x\right) \\
& \quad+f\left(E_{x}^{+2} x_{\mathfrak{N}}\right) g\left(E_{x}^{+} x_{\mathfrak{N}}\right)-f\left(x_{0}\right) g\left(E_{x}^{-} x_{0}\right),
\end{aligned}
$$


(iii) and the parameterization "invariance" property

$$
\begin{aligned}
\int_{x_{0} \leq x_{s} \leq x_{\mathfrak{N}}} \mathbb{D} x f(x) \\
=\int_{x_{0} \leq x_{s} \leq x_{\mathfrak{N}}} \mathbb{D}\left(E_{x}^{+2} x\right) f\left(E_{x}^{+2} x\right) \\
\quad+\Delta y\left(x_{0}\right) f\left(x_{0}\right)-\Delta y\left(E_{x}^{+2} x_{\mathfrak{N}}\right) f\left(E_{x}^{+2} x_{\mathfrak{N}}\right) \\
=\int_{x_{0} \leq x_{s} \leq x_{\mathfrak{N}}} \mathbb{D}\left(E_{x}^{-2} x\right) f\left(E_{x}^{-2} x\right) \\
\quad-\Delta y\left(E_{x}^{-2} x_{0}\right) f\left(E_{x}^{-2} x_{0}\right)+\Delta y\left(x_{\mathfrak{N}}\right) f\left(x_{\mathfrak{N}}\right) .
\end{aligned}
$$

We will apply our theory to the case of the q-quadratic lattice and the Askey-Wilson divided-difference calculus, and in order to simplify the description and to conform to convention we will employ the canonical, that is to say, the centered and symmetrized forms of the lattice and the divided-difference operators. Let us define the base $q=\exp (2 i \eta)$, although we will not restrict ourselves to $q$-domains such as $0<\Re(q)<1$ except to avoid special degenerate cases and to ensure convergence. Consider the projection map from the unit circle $z=e^{i \theta}, \theta \in[-\pi, \pi)$ onto $[-1,1]$ by $x=\frac{1}{2}\left(z+z^{-1}\right)=\cos \theta \in[-1,1]$. We denote the unit circle by $\mathbb{T}$ and the unit open disk by $\mathbb{D}$. The inverse of the projection map defines a two-sheeted Riemann surface, one of which corresponds to the interior of the unit circle, and the other to the exterior. Thus we take the $x$-plane to be cut along $[-1,1]$ and will usually give results for the second sheet, that is, when $|x| \rightarrow \infty$ as $z \rightarrow \infty$. In the symmetrized and canonical form of the lattice we have $\mathcal{A}=\mathcal{C}$, arbitrary and nonzero, $\mathcal{B}=-\cos \eta \mathcal{A}, \mathcal{D}=\mathcal{E}=0, \mathcal{F}=-\sin ^{2} \eta \mathcal{A}$, and $\theta=2 s \eta$. Define the shift operators $E_{x}^{ \pm}$by $E_{x}^{ \pm} f(x)=f\left(\frac{1}{2}\left[q^{1 / 2} z+q^{-1 / 2} z^{-1}\right]\right)$, and set $y_{ \pm}=E_{x}^{ \pm} x$. This implies that

$$
\begin{aligned}
y_{+}+y_{-} & =\left(q^{1 / 2}+q^{-1 / 2}\right) x=2 \cos \eta x \\
\Delta y & :=y_{+}-y_{-}=\frac{1}{2}\left(q^{1 / 2}-q^{-1 / 2}\right)\left(z-z^{-1}\right)=-2 \sin \eta \sin \theta \\
\Delta y^{2} & =\left(q^{1 / 2}-q^{-1 / 2}\right)^{2}\left(x^{2}-1\right) \\
y_{+} y_{-} & =x^{2}+\frac{1}{4}\left(q^{1 / 2}-q^{-1 / 2}\right)^{2}=x^{2}-\sin ^{2} \eta
\end{aligned}
$$


The Askey-Wilson divided-difference operators are defined as

$$
\begin{aligned}
\mathbb{D}_{x} f(x) & =\frac{f\left(\frac{1}{2}\left[q^{1 / 2} z+q^{-1 / 2} z^{-1}\right]\right)-f\left(\frac{1}{2}\left[q^{-1 / 2} z+q^{1 / 2} z^{-1}\right]\right)}{\frac{1}{2}\left(q^{1 / 2}-q^{-1 / 2}\right)\left(z-z^{-1}\right)} \\
\mathbb{M}_{x} f(x) & =\frac{1}{2}\left[f\left(\frac{1}{2}\left[q^{1 / 2} z+q^{-1 / 2} z^{-1}\right]\right)+f\left(\frac{1}{2}\left[q^{-1 / 2} z+q^{1 / 2} z^{-1}\right]\right)\right] .
\end{aligned}
$$

There is an explicit parameterization of the q-quadratic lattice

$$
\begin{aligned}
x(s) & =\frac{1}{2}\left(q^{s}+q^{-s}\right)=\cos (2 \eta s), \\
y_{ \pm}(s) & =\frac{1}{2}\left(q^{s \pm 1 / 2}+q^{-s \mp 1 / 2}\right)=\cos \left(2 \eta\left[s \pm \frac{1}{2}\right]\right) .
\end{aligned}
$$

Here the direct lattice is $G\left[x=\frac{1}{2}\left(a+a^{-1}\right)\right]=\left\{\frac{1}{2}\left(q^{r / 2} a+q^{-r / 2} a^{-1}\right): r \in 2 \mathbb{Z}\right\}$. For $|q|=1$ and $\eta$ not a rational multiple of $\pi$, then the lattice densely fills the interval $[-1,1]$. If $\eta$ is a rational multiple of $\pi$, then one has the root of unity case $q^{N}=1$ and a finite lattice. In the generic case, we will assume that we are dealing with functions $f(x)$ in the class where

$$
\int \mathbb{D} x f(x)=\frac{\sin \eta}{\eta} \int_{-1}^{1} d x f(x)
$$

is applicable. The reader should note that we will not distinguish a function of $x, f(x)$, from the function of $z, \check{f}(z)=f\left(\frac{1}{2}\left[z+z^{-1}\right]\right)$ as done by some authors, and it should be clear from the context which is meant.

\section{$\S 3$. Orthogonal polynomial systems on the nonuniform lattice}

\subsection{General orthogonal polynomial systems}

Our study requires the revision of a number of standard results in orthogonal polynomial theory (see [92], [30], [43]), so we recount our formulation. Let $\left\{l_{n}(x ; a)\right\}_{n=0}^{\infty}$ be a polynomial basis of $L^{2}(w(x) \mathbb{D} x, G)$, where $l_{n}$ is of exact degree $n$ and the support is $G=\left\{E_{x}^{+k} x: k \in 2 \mathbb{Z}\right\}$ or if finite $G=\left\{x_{0}, \ldots, x_{\mathfrak{N}}\right\}$, and where $a$ denotes the set of parameters characterizing the lattice. The appropriate canonical basis is dependent on the lattice type through the general requirements that

(i) $l_{n}$ is of precise degree $n$ so that $l_{n}(x ; a)=g_{n}(a) x^{n}+\mathrm{O}\left(x^{n-1}\right)$ with $g_{n} \neq 0$,

(ii) $\mathbb{D}_{x}$ is an exact lowering operator in this basis 


$$
\mathbb{D}_{x} l_{n}(x ; a)=c_{n}(a) l_{n-1}\left(x ; a^{\prime}\right),
$$

where $c_{n}$ is constant with respect to $x$ and the transformed parameter set $a^{\prime}$ is related to the original $a$ depending on the lattice type.

We will also require the linearization formula $x l_{n}(x ; a)=d_{n}(a) l_{n+1}(x ; a)+$ $e_{n}(a) l_{n}(x ; a)$. A general solution to the two requirements above is the following product expression

$$
l_{n}(x ; a)=g_{n}(a) \prod_{k=0}^{n-1}\left[x-\left(E_{x}^{+}\right)^{2 k} x(a)\right]
$$

where the basal point $x(a)$ is parameterized by $a$. We note that for some lattices the $\operatorname{limit}_{\lim _{n \rightarrow \infty}} l_{n}(x ; a)$ exists and in this case we denote it by $l_{\infty}(x ; a)$. For the classes of quadratic nonuniform lattices the basis choices are tabulated in Table 3.

Consider the general orthogonal polynomial system $\left\{p_{n}(x)\right\}_{n=0}^{\infty}$ defined by the orthogonality relations

$$
\int_{G} \mathbb{D} x w(x) p_{n}(x) l_{m}(x ; b)=\left\{\begin{array}{ll}
0 & 0 \leq m<n, \\
h_{n}(b) & m=n,
\end{array} \quad n \geq 0,\right.
$$

with $G$ denoting the support of the weight $w(x)$. Our system of orthogonal polynomials and their associated functions (to be defined in (3.21)) have a distinguished singular point at $x=\infty$ and possess expansions about this point which can characterize solutions uniquely. This is related to the fact that orthogonal polynomials are the denominators of single-point Padé approximants and that point is conventionally set at $x=\infty$. We give special notation for the coefficients of $x^{n}$ and $x^{n-1}$ in $p_{n}(x)$,

$$
p_{n}(x)=\gamma_{n} x^{n}+\gamma_{n, 1} x^{n-1}+\cdots, \quad n \geq 0 .
$$

The corresponding monic polynomials are then $\pi_{n}(x)=\gamma_{n}^{-1} p_{n}(x)$ given that $n \geq 0$. A consequence of the orthogonality relation is the three-term recurrence relation

$$
a_{n+1} p_{n+1}(x)=\left(x-b_{n}\right) p_{n}(x)-a_{n} p_{n-1}(x), \quad n \geq 0,
$$

and we consider the set of orthogonal polynomials with initial values $p_{-1}=0$ and $p_{0}=\gamma_{0}$. The three-term recurrence coefficients are related to the leading and subleading polynomial coefficients by [92] and [30]

$$
a_{n}=\frac{\gamma_{n-1}}{\gamma_{n}}, \quad b_{n}=\frac{\gamma_{n, 1}}{\gamma_{n}}-\frac{\gamma_{n+1,1}}{\gamma_{n+1}}, \quad n \geq 1 .
$$


Table 3: Canonical bases for the nonuniform lattices of quadratic type.

\begin{tabular}{|c|c|c|c|}
\hline DDO & Lattice type & Basis $l_{r}$ & Notes \\
\hline$\frac{d}{d x}$ & continuous & $x^{r}$ & $c_{r}=r$ \\
\hline$\Delta_{x}$ & linear & $x^{(r)}=\prod_{k=0}^{r-1}(x-k)=\frac{\Gamma(x+1)}{\Gamma(x-r+1)}$ & $c_{r}=r$ \\
\hline$D_{q}$ & $q$-linear & $\begin{aligned}(a x ; q)_{r} & =\prod_{k=0}^{r-1}\left(1-a q^{k} x\right) \\
& =\frac{(a x ; q)_{\infty}}{\left(a q^{r} x ; q\right)_{\infty}}\end{aligned}$ & $\begin{aligned} c_{r} & =-\frac{1-a q^{r}}{q-1} \\
a^{\prime} & =q a\end{aligned}$ \\
\hline$W$ & quadratic & $\begin{array}{l}\prod_{k=0}^{r-1}\left[x+(k+a)^{2}\right] \\
\quad=\frac{\Gamma(r+a-i \sqrt{x}) \Gamma(r+a+i \sqrt{x})}{\Gamma(a-i \sqrt{x}) \Gamma(a+i \sqrt{x})}\end{array}$ & $\begin{array}{l}c_{r}=r \\
a^{\prime}=a+\frac{1}{2}\end{array}$ \\
\hline $\mathbb{D}_{x}$ & $q$-quadratic & $\begin{array}{l}\left(a z, a z^{-1} ; q\right)_{r} \\
\quad=\frac{\left(a z, a z^{-1} ; q\right)_{\infty}}{\left(a q^{r} z, a q^{r} z^{-1} ; q\right)_{\infty}}\end{array}$ & $\begin{array}{l}c_{r}=-2 a \frac{q^{r}-1}{q-1} \\
a^{\prime}=q^{1 / 2} a \\
g_{r}=(-2 a)^{r} q^{\frac{1}{2} r(r-1)} \\
d_{r}=-\frac{1}{2 a q^{r}} \\
e_{r}=\frac{1}{2}\left(a q^{r}+a^{-1} q^{-r}\right)\end{array}$ \\
\hline
\end{tabular}

The initial values of the recurrence coefficients are

$$
b_{0}=-\frac{\gamma_{1,1}}{\gamma_{1}}, \quad \gamma_{0,1}=0
$$

where $a_{0}$ is not fixed by the initial polynomials but rather by the initial associated functions (see after (3.22)). 
The orthogonality relation (3.3) is derived from the linear functional on the space of polynomials $\mathcal{L}: p \in \Pi \mapsto \mathbb{C}$, and we employ our basis polynomials as an expansion basis although not necessarily with the same parameter as above

$$
p_{n}(x)=\sum_{k=0}^{n} c_{n, k}(a) l_{k}(x ; a) \quad n \geq 0
$$

Consequently, we define the moments $\left\{m_{j, k}\right\}_{j, k=0,1, \ldots, \infty}$ of the weight as the action of this functional on products of the basis polynomials, defined as

$$
m_{j, k}(b, a):=\int_{G} \mathbb{D} x w(x) l_{j}(x ; b) l_{k}(x ; a), \quad j, k \geq 0 .
$$

Central objects in our theory are the moment determinants

$$
\Delta_{n}:=\operatorname{det}\left[m_{j, k}\right]_{j, k=0, \ldots, n-1}, \quad n \geq 1, \Delta_{0}:=1,
$$

and

$$
\begin{gathered}
\Sigma_{n, j}:=\operatorname{det}\left(\begin{array}{ccccccc}
m_{0,0} & \cdots & m_{0, j-1} & {[]} & m_{0, j+1} & \cdots & m_{0, n} \\
\vdots & \vdots & \vdots & \vdots & \vdots & \cdots & \vdots \\
m_{n-1,0} & \cdots & m_{n-1, j-1} & {[]} & m_{n-1, j+1} & \cdots & m_{n-1, n}
\end{array}\right), \\
n \geq 1, j=0, \ldots, n-1
\end{gathered}
$$

defined in terms of the moments above. Obviously $\Delta_{n}=\Sigma_{n, n}$, and we set $\Sigma_{0,0}:=0$. The expansion coefficients are given in terms of these determinants

$$
c_{n, j}(a)=(-)^{n+j} h_{n}(b) \frac{\Sigma_{n, j}}{\Delta_{n+1}}, \quad c_{n, n}(a)=h_{n}(b) \frac{\Delta_{n}}{\Delta_{n+1}} .
$$

It follows from (3.3) that

$$
\int_{G} \mathbb{D} x w(x)\left[p_{n}(x)\right]^{2}=c_{n, n}(b) h_{n}(b), \quad n \geq 0,
$$

and thus for $p_{n}(x)$ to be normalized as well as orthogonal, we set $c_{n, n}(a) h_{n}(a)=1$. We have moment determinant representations of the polynomials 


$$
p_{n}(x)
$$

$$
=\frac{c_{n, n}(a)}{\Delta_{n}} \operatorname{det}\left(\begin{array}{ccccc}
m_{0,0} & \cdots & m_{0, j} & \cdots & m_{0, n} \\
\vdots & \vdots & \vdots & \cdots & \vdots \\
m_{n-1,0} & \cdots & m_{n-1, j} & \cdots & m_{n-1, n} \\
l_{0} & \cdots & l_{j} & \cdots & l_{n}
\end{array}\right), \quad n \geq 0 .
$$

The three-term recurrence coefficients are related to these determinants

$$
\begin{aligned}
a_{n}^{2} & =d_{n-1}(a) d_{n-1}(b) \frac{\Delta_{n+1} \Delta_{n-1}}{\Delta_{n}^{2}}, \quad n \geq 1, \\
b_{n} & =e_{n}(a)+d_{n}(a) \frac{\Sigma_{n+1, n}}{\Delta_{n+1}}-d_{n-1}(a) \frac{\Sigma_{n, n-1}}{\Delta_{n}}, \quad n \geq 0, \\
\gamma_{n}^{2} & =g_{n}(a) g_{n}(b) \frac{\Delta_{n}}{\Delta_{n+1}}, \quad n \geq 0,
\end{aligned}
$$

where each coefficient is independent of the choices of $a, b$ as can be easily verified from their determinantal definitions given previously.

Another set of polynomial solutions to the three-term recurrence relation are the associated polynomials $\left\{p_{n}^{(1)}(x)\right\}_{n=0}^{\infty}$, defined by

$$
p_{n-1}^{(1)}(x):=\int_{G} \mathbb{D} y w(y) \frac{p_{n}(y)-p_{n}(x)}{y-x}, \quad n \geq 0 .
$$

In particular, these polynomials satisfy

$$
a_{n+1} p_{n}^{(1)}(x)=\left(x-b_{n}\right) p_{n-1}^{(1)}(x)-a_{n} p_{n-2}^{(1)}(x),
$$

with the initial conditions $p_{-1}^{(1)}(x)=0, p_{0}^{(1)}(x)=m_{0,0} \gamma_{1}$. Note the shift by one decrement in comparison to the three-term recurrence (3.5) for the polynomials $\left\{p_{n}(x)\right\}_{n=0}^{\infty}$. We also need the definition of the Stieltjes function

$$
f(x) \equiv \int_{G} \mathbb{D} y \frac{w(y)}{x-y}, \quad x \notin G,
$$

which is a moment-generating function in the sense

$$
f(x)=\frac{f_{\infty}(x ; a)}{l_{\infty}(x ; a)}+\sum_{n=0}^{\infty} \frac{m_{0, n}(a)}{d_{n}(a) l_{n+1}(x ; a)}, \quad x \notin G, x \rightarrow \infty,
$$

and that splits into two parts - one part being a series with inverse basis polynomials and the other part a remainder, which may be absent for some 
lattices. We define nonpolynomial associated functions or functions of the second kind $\left\{q_{n}(x)\right\}_{n=0}^{\infty}$ by

$$
q_{n}(x):=f(x) p_{n}(x)-p_{n-1}^{(1)}(x), \quad n \geq 0,
$$

which also satisfy the three-term recurrence relation (3.5), namely,

$$
a_{n+1} q_{n+1}(x)=\left(x-b_{n}\right) q_{n}(x)-a_{n} q_{n-1}(x), \quad n \geq 0,
$$

subject to the initial values $q_{-1}(x)=1 / a_{0} \gamma_{0}, q_{0}(x)=\gamma_{0} f(x)$. The initial value of $a_{0}$ is irrelevant and therefore arbitrary in so far as the polynomials are concerned, however many relations for the whole system extend from $n \geq 1$ to include $n=0$ if we allow this to be finite, nonzero, and satisfying the above initial condition. The associated functions also have a determinantal representation

$$
\begin{aligned}
& q_{n}(x) \\
& =\frac{c_{n, n}(a)}{\Delta_{n}} \operatorname{det}\left(\begin{array}{ccccc}
m_{0,0} & \cdots & m_{0, j} & \cdots & m_{0, n} \\
\vdots & \vdots & \vdots & \cdots & \vdots \\
m_{n-1,0} & \cdots & m_{n-1, j} & \cdots & m_{n-1, n} \\
f_{0} & \cdots & f_{j} & \cdots & f_{n}
\end{array}\right), \quad n \geq 0,
\end{aligned}
$$

where

$$
f_{j}(x ; a):=\int_{G} \mathbb{D} y w(y) \frac{l_{j}(y ; a)}{x-y}, \quad j \geq 1, f_{0}=g_{0} f .
$$

Likewise, this function has an expansion analogous to (3.20)

$$
f_{j}(x ; b)=\frac{f_{\infty, j}(x ; b)}{l_{\infty}(x ; a)}+\sum_{n=0}^{\infty} \frac{m_{j, n}(b, a)}{d_{n}(a) l_{n+1}(x ; a)}, \quad x \notin G, x \rightarrow \infty .
$$

The polynomials and their associated functions satisfy the Casoratian relation

$$
p_{n}(x) q_{n-1}(x)-p_{n-1}(x) q_{n}(x)=\frac{1}{a_{n}}, \quad n \geq 0 .
$$

Central to our analysis is a composite of polynomial and nonpolynomial solutions of (3.5), the $2 \times 2$ matrix variable

$$
Y_{n}(x)=\left(\begin{array}{cc}
p_{n}(x) & \frac{q_{n}(x)}{w(x)} \\
p_{n-1}(x) & \frac{q_{n-1}(x)}{w(x)}
\end{array}\right), \quad n \geq 0 .
$$


We will refer to this as the orthogonal polynomial system (OPS). From (3.26) we note that

$$
\operatorname{det} Y_{n}(x)=\frac{1}{a_{n} w(x)}, \quad n \geq 0
$$

The three-term recurrence is then recast as the matrix equation

$$
Y_{n+1}(x)=K_{n} Y_{n}(x), \quad n \geq 0,
$$

with the recurrence matrix given by

$$
K_{n}(x)=\frac{1}{a_{n+1}}\left(\begin{array}{cc}
x-b_{n} & -a_{n} \\
a_{n+1} & 0
\end{array}\right), \quad \operatorname{det} K_{n}=\frac{a_{n}}{a_{n+1}}, \quad n \geq 0 .
$$

A well-known consequence of (3.5) are the Christoffel-Darboux summation formulas

$$
\begin{aligned}
& \sum_{j=0}^{n-1} p_{j}(x) p_{j}(y) \\
& \quad=a_{n} \frac{\left[p_{n}(x) p_{n-1}(y)-p_{n-1}(x) p_{n}(y)\right]}{x-y}, \quad n \geq 0, \\
& \sum_{j=0}^{n-1} q_{j}(x) p_{j}(y) \\
& \quad=a_{n} \frac{\left[q_{n}(x) p_{n-1}(y)-q_{n-1}(x) p_{n}(y)\right]}{x-y}+\frac{1}{x-y}, \quad n \geq 0, \\
& \sum_{j=0}^{n-1} q_{j}(x) q_{j}(y) \\
& \quad=a_{n} \frac{\left[q_{n}(x) q_{n-1}(y)-q_{n-1}(x) q_{n}(y)\right]}{x-y}-\frac{f(x)-f(y)}{x-y}, \quad n \geq 0 .
\end{aligned}
$$

If one is only interested in the leading orders of the large $x$ expansion rather than a systematic expansion, then it is convenient to employ an expansion in monomials rather than in basis functions. Extending (3.4) we 
have expansions about the fixed singularity at $x=\infty$

$$
\begin{aligned}
p_{n}(x)= & \gamma_{n}\left[x^{n}-\left(\sum_{i=0}^{n-1} b_{i}\right) x^{n-1}\right. \\
& \left.+\left(\sum_{0 \leq i<j<n} b_{i} b_{j}-\sum_{i=1}^{n-1} a_{i}^{2}\right) x^{n-2}+\mathrm{O}\left(x^{n-3}\right)\right]
\end{aligned}
$$

valid for $n \geq 1$, while for the associated functions

$$
\begin{aligned}
q_{n}(x)= & \gamma_{n}^{-1}\left[x^{-n-1}+\left(\sum_{i=0}^{n} b_{i}\right) x^{-n-2}\right. \\
& \left.+\left(\sum_{0 \leq i \leq j \leq n} b_{i} b_{j}+\sum_{i=1}^{n+1} a_{i}^{2}\right) x^{-n-3}+\mathrm{O}\left(x^{-n-4}\right)\right]
\end{aligned}
$$

valid for $n \geq 0$.

\section{2. $q$-Quadratic lattice}

In Sections 5 and 7 we intend to apply our theory to the $q$-quadratic lattice and will draw upon numerous properties of the corresponding basis, which we discuss here. First, we recall the analytic continuation of $\phi_{n}(x ; a)$, which is

$$
\phi_{r}(x ; a)=\frac{\left(a z, a z^{-1} ; q\right)_{\infty}}{\left(a q^{r} z, a q^{r} z^{-1} ; q\right)_{\infty}}
$$

for all $r \in \mathbb{C}$ but subject to $|q|<1$. We will employ the shorthand notation $\left(a z^{ \pm 1} ; q\right)_{\infty}=\left(a z, a z^{-1} ; q\right)_{\infty}$. Implicit in the above formula is the elliptic-like function $\phi_{\infty}(x ; a)$ which has meaning for all $a, x \in \mathbb{C}$ for $|q|<1$. The actions of the divided-difference operators are

$$
\begin{aligned}
\mathbb{D}_{x} \phi_{r}(x ; a)= & 2 a \frac{1-q^{r}}{q-1} \phi_{r-1}\left(x ; q^{1 / 2} a\right), \\
\mathbb{M}_{x} \phi_{r}(x ; a)= & \frac{1}{2}\left(1+q^{-r}\right) \phi_{r}\left(x ; q^{1 / 2} a\right) \\
& +\frac{1}{2}\left(1-q^{-r}\right)\left(1-a^{2} q^{2 r-1}\right) \phi_{r-1}\left(x ; q^{1 / 2} a\right), \\
\mathbb{D}_{x} \phi_{\infty}(x ; a)= & \frac{2 a}{q-1} \phi_{\infty}\left(x ; q^{1 / 2} a\right),
\end{aligned}
$$




$$
\mathbb{M}_{x} \phi_{\infty}(x ; a)=\phi_{\infty}\left(x ; q^{-1 / 2} a\right)
$$

The expansion theorem of Ismail [40, Theorem 1.3] (see also [41]) states the following.

Proposition 3.1. Let $p$ be a polynomial of degree $n$. Then

$$
p(x)=\sum_{k=0}^{n} p_{k} \phi_{k}(x ; a)
$$

for any $a \in \mathbb{C}$, where

$$
p_{k}=\frac{(q-1)^{k}}{(2 a)^{k}(q ; q)_{k}} q^{-k(k-1) / 4}\left(\mathbb{D}_{x}^{k} p\right)\left(x_{k}\right)
$$

with $x_{k}=\frac{1}{2}\left(q^{k / 2} a+q^{-k / 2} a^{-1}\right)$.

In these works (see (2.2) and [40, Proof of Theorem 1.1]), we also have the change of base formula

$$
\phi_{n}(x ; b)=\sum_{k=0}^{n}\left[\begin{array}{l}
n \\
k
\end{array}\right]_{q}\left(a b q^{k}, b / a ; q\right)_{n-k}\left(\frac{b}{a}\right)^{k} \phi_{k}(x ; a),
$$

where we use the standard definition of the $q$-binomial coefficient. This result allows us to derive the following linearization formula.

LEMMA 3.1. The product of two basis polynomials with the same base a has the following expansion in terms of the same basis

$$
\begin{aligned}
& \phi_{k}(x ; a) \phi_{l}(x ; a) \\
& =(-)^{k+l} q^{-k l} \sum_{m=\max (k, l)}^{k+l}(-)^{m}\left(a^{2} q^{m} ; q\right)_{k+l-m} \\
& \quad \times \frac{(q ; q)_{l}(q ; q)_{k}}{(q ; q)_{k+l-m}(q ; q)_{m-k}(q ; q)_{m-l}} \phi_{m}(x ; a),
\end{aligned}
$$

for all $k, l \in \mathbb{N}$.

Lastly, we have the Cauchy expansion formula of Ismail and Stanton [47, Theorem 2.1]. 
Proposition 3.2. The Cauchy kernel has the expansion

$$
\frac{1}{y-x}=\frac{1}{y-x} \frac{\phi_{\infty}(x ; a)}{\phi_{\infty}(y ; a)}-2 a \sum_{n=0}^{\infty} \frac{\phi_{n}(x ; a)}{\phi_{n+1}(y ; a)} q^{n}
$$

for all $y$ such that $y \neq x$ and $\phi_{\infty}(y ; a) \neq 0$. The expansion also holds for $y=y_{0}$ with $\phi_{\infty}\left(y_{0} ; a\right)=0$ but $y_{0} \neq x$ in the sense that the left-hand side $\left(y_{0}-x\right)^{-1}$ equals the limit of the right-hand side as $y \rightarrow y_{0}$.

This implies the following expansion of the Stieltjes function.

Corollary 3.1. The Stieltjes function has the following expansion as $x \rightarrow \infty$ with $x \neq x_{k}(a)$, with $k \in 2 \mathbb{Z}$ (i.e., $\phi_{\infty}(x ; a) \neq 0$ )

$$
f(x)=\frac{f_{\infty}(x)}{\phi_{\infty}(x ; a)}-2 a \sum_{n=0}^{\infty} \frac{q^{n}}{\phi_{n+1}(x ; a)} m_{0, n}(a),
$$

where

$$
f_{\infty}(x)=\int_{G} \mathbb{D} y w(y) \frac{\phi_{\infty}(y ; a)}{x-y} .
$$

Conforming with standard notation (see [31]), we define the basic hypergeometric function ${ }_{r+1} \varphi_{r}$ by the series

$$
{ }_{r+1} \varphi_{r}\left[\begin{array}{cccc}
a_{1}, & a_{2}, & \ldots, & a_{r+1} \\
b_{1}, & b_{2}, & \ldots, & b_{r}
\end{array} ; q, z\right]=\sum_{n=0}^{\infty} \frac{\left(a_{1}, a_{2}, \ldots, a_{r+1} ; q\right)_{n}}{\left(q, b_{1}, b_{2}, \ldots, b_{r} ; q\right)_{n}} z^{n}
$$

which is convergent for $|z|<1$. The very-well-poised basic hypergeometric function ${ }_{r+1} W_{r}$ is a specialization of the above

$$
\begin{aligned}
& { }_{r+1} W_{r}\left(a_{1} ; a_{4}, a_{5}, \ldots, a_{r+1} ; q, z\right) \\
& ={ }_{r+1} \varphi_{r}\left[\begin{array}{cccccc}
a_{1}, & q \sqrt{a_{1}}, & -q \sqrt{a_{1}}, & a_{4}, & \ldots, & a_{r+1} \\
\sqrt{a_{1}}, & -\sqrt{a_{1}}, & q a_{1} / a_{4}, & \ldots, & q a_{1} / a_{r+1} &
\end{array}\right]
\end{aligned}
$$

so that $q a_{1}=a_{2} b_{1}=a_{3} b_{2}=\cdots=a_{r+1} b_{r}$ and $a_{2}=q \sqrt{a_{1}}, a_{3}=-q \sqrt{a_{1}}$. The ${ }_{r+1} \varphi_{r}$ or ${ }_{r+1} W_{r}$ functions may also be balanced, whereby $z=q$ and $\prod_{j=1}^{r} b_{j}=$ $q \prod_{j=1}^{r+1} a_{j}$. 


\section{$\S 4$. Spectral differences}

In this section we lay out the structures of the spectral divided-difference operator in the context of the orthogonal polynomial system for a general lattice type. Our starting point is the notion of the $\mathbb{D}$-semiclassical weight, as given by the following definition of Magnus.

Definition $4.1([60,(2.7)])$. Let the $\mathbb{D}$-semiclassical weight satisfy

$$
W \mathbb{D}_{x} w=2 V \mathbb{M}_{x} w
$$

or equivalently

$$
\frac{w\left(y_{+}\right)}{w\left(y_{-}\right)}=\frac{W+\Delta y V}{W-\Delta y V}(x)
$$

for $W(x), V(x)$ irreducible polynomials, which we will call spectral data polynomials. Furthermore, we assume that $W \pm \Delta y V \neq 0$ for all $x \in G$. For minimal degrees of $W, V$, this is the analogue of the Pearson equation.

REMARK 4.1. On the finite lattice $x \in\left\{x_{0}, \ldots, x_{\mathfrak{N}}\right\}$ we naturally require $w(x) \neq 0$, however we will impose upper and lower terminating conditions

$$
w\left(E_{x}^{+2} x_{\mathfrak{N}}\right)=w\left(E_{x}^{-2} x_{0}\right)=0,
$$

respectively. These are the conditions analogous to Nikiforov, Suslov, and Uvarov [78, (2.3.2), (3.3.3)]. To be consistent with (4.2), this implies that

$$
(W+\Delta y V)\left(E_{x}^{+} x_{\mathfrak{N}}\right)=(W-\Delta y V)\left(E_{x}^{-} x_{0}\right)=0 .
$$

REMARK 4.2. In a series of works, Suslov and collaborators (see [5], [91], [6], [84], [83]) have studied the Pearson equation for all of the lattices admissible in the classification, and sought solutions for the weight functions given suitable polynomials for $W, V$. However, they limited their choices to examples of minimal degree for $W, V$ which made contact with those weights found in the Askey table. In terms of our own variables, those found in [91] are given by

$$
\begin{aligned}
\sigma & =E_{x}^{-}(W-\Delta y V), \\
\tau & =\frac{E_{x}^{+}(W+\Delta y V)(x)-E_{x}^{-}(W-\Delta y V)(x)}{\Delta y} .
\end{aligned}
$$

From (3.20) we recognize $f(x)$ as a moment-generating function, and a key element in our theory are the systems of linear divided-difference 
equations satisfied by the moments. Before we state such systems, we need to note the following result.

Lemma 4.1. Let $l_{k}(x ; a)$ be a canonical basis polynomial. For any $k \in \mathbb{Z}_{\geq 0}$ and $a \in \mathbb{C}$, the weight satisfies the integral equation

$$
\begin{gathered}
\int_{x_{0} \leq x \leq x_{\mathfrak{N}}} \mathbb{D} x \frac{w(x)}{\Delta y(x)}\left\{\left(l_{k}[W+\Delta y V]\right)\left(E_{x}^{+} x\right)\right. \\
\left.-\left(l_{k}[W-\Delta y V]\right)\left(E_{x}^{-} x\right)\right\}=0 .
\end{gathered}
$$

Proof. We will prove this statement accounting for the boundary terms explicitly. We establish two preliminary identities first, namely

$$
\begin{aligned}
& \int_{x_{0} \leq x \leq x_{\mathfrak{N}}} \mathbb{D} x w(x)\left\{\frac{1}{E_{x}^{+} \Delta y} E_{x}^{+}\left(l_{k}[W+\Delta y V]\right)\right. \\
& \left.-\frac{E_{x}^{-2} \Delta y}{\Delta y\left(E_{x}^{-} \Delta y\right)} E_{x}^{-}\left(l_{k}[W-\Delta y V]\right)\right\}=0, \\
& \int_{x_{0} \leq x \leq x_{\mathfrak{N}}} \mathbb{D} x w(x)\left\{\frac{E_{x}^{+2} \Delta y}{\Delta y\left(E_{x}^{+} \Delta y\right)} E_{x}^{+}\left(l_{k}[W+\Delta y V]\right)\right. \\
& \left.-\frac{1}{E_{x}^{-} \Delta y} E_{x}^{-}\left(l_{k}[W-\Delta y V]\right)\right\}=0,
\end{aligned}
$$

with $l_{k}:=l_{k}(x ; a)$. Taking the first of these, we compute

$$
\begin{aligned}
0= & \int_{x_{0} \leq x \leq x_{\mathfrak{N}}} \mathbb{D} x l_{k}\left(E_{x}^{+} x\right)\left(W \mathbb{D}_{x} w-2 V M_{x} w\right)\left(E_{x}^{+} x\right) \\
= & \sum_{0 \leq s \leq \mathfrak{N}} \Delta y\left(x_{s}\right) l_{k}\left(E_{x}^{+} x_{s}\right) W\left(E_{x}^{+} x_{s}\right) \frac{w\left(E_{x}^{+2} x_{s}\right)-w\left(x_{s}\right)}{\Delta y\left(E_{x}^{+} x_{s}\right)} \\
& -\sum_{0 \leq s \leq \mathfrak{N}} \Delta y\left(x_{s}\right) l_{k}\left(E_{x}^{+} x_{s}\right) V\left(E_{x}^{+} x_{s}\right)\left[w\left(E_{x}^{+2} x_{s}\right)+w\left(x_{s}\right)\right] \\
= & \sum_{0 \leq s \leq \mathfrak{N}} \frac{\Delta y\left(E_{x}^{-2} x_{s}\right)}{\Delta y\left(E_{x}^{-} x_{s}\right)} l_{k}\left(E_{x}^{-} x_{s}\right) W\left(E_{x}^{-} x_{s}\right) w\left(x_{s}\right) \\
& -\sum_{0 \leq s \leq \mathfrak{N}} \frac{\Delta y\left(x_{s}\right)}{\Delta y\left(E_{x}^{+} x_{s}\right)} l_{k}\left(E_{x}^{+} x_{s}\right) W\left(E_{x}^{+} x_{s}\right) w\left(x_{s}\right) \\
& +\frac{\Delta y\left(x_{\mathfrak{N}}\right)}{\Delta y\left(E_{x}^{+} x_{\mathfrak{N}}\right)} l_{k}\left(E_{x}^{+} x_{\mathfrak{N}}\right) W\left(E_{x}^{+} x_{\mathfrak{N}}\right) w\left(E_{x}^{+2} x_{\mathfrak{N}}\right)
\end{aligned}
$$




$$
\begin{aligned}
& -\frac{\Delta y\left(E_{x}^{-2} x_{0}\right)}{\Delta y\left(E_{x}^{-} x_{0}\right)} l_{k}\left(E_{x}^{-} x_{0}\right) W\left(E_{x}^{-} x_{0}\right) w\left(x_{0}\right) \\
& -\sum_{0 \leq s \leq \mathfrak{N}} \Delta y\left(E_{x}^{-2} x_{s}\right) l_{k}\left(E_{x}^{-} x_{s}\right) V\left(E_{x}^{-} x_{s}\right) w\left(x_{s}\right) \\
& -\sum_{0 \leq s \leq \mathfrak{N}} \Delta y\left(x_{s}\right) l_{k}\left(E_{x}^{+} x_{s}\right) V\left(E_{x}^{+} x_{s}\right) w\left(x_{s}\right) \\
& -\Delta y\left(x_{\mathfrak{N}}\right) l_{k}\left(E_{x}^{+} x_{\mathfrak{N}}\right) V\left(E_{x}^{+} x_{\mathfrak{N}}\right) w\left(E_{x}^{+2} x_{\mathfrak{N}}\right) \\
& +\Delta y\left(E_{x}^{-2} x_{0}\right) l_{k}\left(E_{x}^{-} x_{0}\right) V\left(E_{x}^{-} x_{0}\right) w\left(x_{0}\right) \\
& =\sum_{0 \leq s \leq \mathfrak{N}} \Delta y\left(x_{s}\right) w\left(x_{s}\right)\left\{-\left(l_{k}\left[\frac{W}{\Delta y}+V\right]\right)\left(E_{x}^{+} x_{s}\right)\right. \\
& \left.+\frac{\Delta y\left(E_{x}^{-2} x_{s}\right)}{\Delta y\left(x_{s}\right)}\left(l_{k}\left[\frac{W}{\Delta y}-V\right]\right)\left(E_{x}^{-} x_{s}\right)\right\} \\
& +\frac{\Delta y\left(x_{\mathfrak{N}}\right)}{\Delta y\left(E_{x}^{+} x_{\mathfrak{N}}\right)} l_{k}\left(E_{x}^{+} x_{\mathfrak{N}}\right) w\left(E_{x}^{+2} x_{\mathfrak{N}}\right)[W-\Delta y V]\left(E_{x}^{+} x_{\mathfrak{N}}\right) \\
& +\frac{\Delta y\left(E_{x}^{-2} x_{0}\right)}{\Delta y\left(E_{x}^{-} x_{0}\right)} l_{k}\left(E_{x}^{-} x_{0}\right) w\left(x_{0}\right)[W-\Delta y V]\left(E_{x}^{-} x_{0}\right),
\end{aligned}
$$

where the boundary terms arise from using the integration by parts and the change of variables $x_{s} \mapsto E_{x}^{ \pm 2} x_{s}$ formulas. Both boundary terms are in fact zero due to the upper and lower terminating conditions. The second formula (4.8) is just a variation of the first with the integrand evaluated at $E_{x}^{-} x_{s}$ instead. Now for a general quadratic lattice

$$
\frac{\Delta y+E_{x}^{ \pm 2} \Delta y}{E_{x}^{ \pm} \Delta y}=-2 \frac{\mathcal{B}}{\mathcal{A}},
$$

so we simply add (4.7) and (4.8) and note that a common, constant factor appears in the integrand of precisely this form. Then (4.6) immediately follows.

REMARK 4.3. An equivalent system for general lattices has been derived by Suslov [91] in the special case of $k=0$ of (4.6). 
We also need to express $W \pm \Delta y V$ in terms of canonical basis polynomials and formulate the following definitions for the coefficients $\kappa_{N, l}(a), \delta_{N, l}(a)$ :

$$
\begin{aligned}
& E_{x}^{+}(W+\Delta y V)(x)+E_{x}^{-}(W-\Delta y V)(x)=: 2 \sum_{k=0}^{N} \kappa_{N, k}(a) l_{k}(x ; a), \\
& E_{x}^{+}(W+\Delta y V)(x)-E_{x}^{-}(W-\Delta y V)(x)=: \Delta y \sum_{k=0}^{N-1} \delta_{N, k}(a) l_{k}(x ; a) .
\end{aligned}
$$

Here $N$ is the cutoff determined by the degrees of $W, V$ and the lattice type.

COROLlary 4.1. Let us assume that $b^{\prime} \neq a$, where $b, b^{\prime}$ are related as $a, a^{\prime}$ are by (3.1). For all $k \in \mathbb{Z}_{\geq 0}$, the moments $m_{k, l}\left(b^{\prime}, a\right)$ are characterized by the linear, homogeneous recurrence relations

$$
\begin{aligned}
& {\left[d_{k}(b) c_{k+1}(b)+\frac{\mathcal{B}}{\mathcal{A}} d_{k-1}\left(b^{\prime}\right) c_{k}(b)\right] \sum_{l=0}^{N-1} \delta_{N, l}(a) m_{k, l}\left(b^{\prime}, a\right)} \\
& \quad+c_{k}(b) \sum_{l=0}^{N}\left[\kappa_{N, l}(a)+\left(e_{k}(b)+\frac{\mathcal{B}}{\mathcal{A}} e_{k-1}\left(b^{\prime}\right)+\frac{\mathcal{D}}{\mathcal{A}}\right) \delta_{N, l}(a)\right] \\
& \quad \times m_{k-1, l}\left(b^{\prime}, a\right)=0 .
\end{aligned}
$$

For $k=0$, the last term is absent and therefore $b, b^{\prime}$ do not appear.

Proof. We start with the integral formula (4.6) and employ the resolutions of $E_{x}^{+}(W+\Delta y V)$ and $E_{x}^{-}(W-\Delta y V)$ as given by (4.9) and (4.10). In addition, we require expressions for $\mathbb{D}_{x} l_{k}(x ; b)$ and $\mathbb{M}_{x} l_{k}(x ; b)$, and so we use (3.1) for the former and

$$
\begin{aligned}
\mathbb{M}_{x} l_{k}(x ; b) & \\
= & \left(d_{k}(b) c_{k+1}(b)+\frac{\mathcal{B}}{\mathcal{A}} d_{k-1}\left(b^{\prime}\right) c_{k}(b)\right) l_{k}\left(x ; b^{\prime}\right) \\
& +c_{k}(b)\left(e_{k}(b)+\frac{\mathcal{B}}{\mathcal{A}} e_{k-1}\left(b^{\prime}\right)+\frac{\mathcal{D}}{\mathcal{A}}\right) l_{k-1}\left(x ; b^{\prime}\right)
\end{aligned}
$$

for the latter.

If a specialization of the parameters $b^{\prime}, a$ is made to effect some cancellation with corresponding factors in the weight, then the moments are given by an integral whose integrand has the same structure, and (4.11) is now 
a linear divided-difference equation with respect to the internal parameters of the weight.

Now we continue to develop the consequences of the $\mathbb{D}$-semiclassical weight on the orthogonal polynomial system, and in particular for the Stieltjes function. The following result will be crucial for our approach.

Proposition 4.1. Given a $\mathbb{D}$-semiclassical class of an orthogonal polynomial system fulfilling (4.1) and the conditions therein, then the Stieltjes function satisfies

$$
W \mathbb{D}_{x} f=2 V \mathbb{M}_{x} f+U
$$

where $U(x)$ in (4.13) is a polynomial in $x$ (as are $V(x)$ and $W(x)$ ).

Proof. Starting with the definition (3.19), we compute

$$
\mathbb{D}_{x} f=\int_{u_{0} \leq u \leq u_{\mathfrak{N}}} \mathbb{D} u w(u) \mathbb{D}_{x} \frac{1}{x-u},
$$

and make the evaluation of the factor

$$
\mathbb{D}_{x} \frac{1}{x-u}=-\frac{1}{\left(E_{x}^{+} x-u\right)\left(E_{x}^{-} x-u\right)} .
$$

However, we note that

$$
\mathbb{D}_{u} \frac{1}{x-u}=\frac{1}{\left(x-E_{u}^{+} u\right)\left(x-E_{u}^{-} u\right)},
$$

so that

$$
\mathbb{D}_{x} \frac{1}{x-u}=-\frac{\left(x-E_{u}^{+} u\right)\left(x-E_{u}^{-} u\right)}{\left(E_{x}^{+} x-u\right)\left(E_{x}^{-} x-u\right)} \mathbb{D}_{u} \frac{1}{x-u}=-\mathbb{D}_{u} \frac{1}{x-u},
$$

where we have assumed a symmetric quadratic lattice for simplicity, but point out that our argument could be extended to the general case. We deduce then that

$$
\begin{aligned}
\mathbb{D}_{x} f & =-\int_{u_{0} \leq u \leq u_{\mathfrak{N}}} \mathbb{D} u w(u) \mathbb{D}_{u} \frac{1}{x-u} \\
& =\int_{u_{0} \leq u \leq u_{\mathfrak{N}}} \mathbb{D}\left(E_{u}^{+} u\right) \frac{1}{x-E_{u}^{+} u} \mathbb{D}_{u} w\left(E_{u}^{+} u\right)-\frac{w\left(E_{u}^{+2} u_{\mathfrak{N}}\right)}{x-E_{u}^{+} u_{\mathfrak{N}}}+\frac{w\left(u_{0}\right)}{x-E_{u}^{-} u_{0}},
\end{aligned}
$$


after employing the formula for integration by parts. We next use the definition of the $\mathbb{D}$-semiclassical weight (4.1) and perform a sequence of subtractions in the numerator of the integrand

$$
\begin{aligned}
W(x) & \mathbb{D}_{x} f(x) \\
= & \int_{u_{0} \leq u \leq u_{\mathfrak{N}}} \mathbb{D}\left(E_{u}^{+} u\right) \frac{1}{x-E_{u}^{+} u} \mathbb{M}_{u} w\left(E_{u}^{+} u\right) \frac{2 V\left(E_{u}^{+} u\right)}{W\left(E_{u}^{+} u\right)} W(x) \\
& -\frac{w\left(E_{u}^{+2} u_{\mathfrak{N}}\right)}{x-E_{u}^{+} u_{\mathfrak{N}}} W(x)+\frac{w\left(u_{0}\right)}{x-E_{u}^{-} u_{0}} W(x) \\
= & 2 V(x) \mathbb{M}_{x} f(x)+2 V(x)\left\{\int_{u_{0} \leq u \leq u_{\mathfrak{N}}} \mathbb{D}\left(E_{u}^{+} u\right) \frac{\mathbb{M}_{u} w\left(E_{u}^{+} u\right)}{x-E_{u}^{+} u}-\mathbb{M}_{x} f(x)\right\} \\
& +2 \int_{u_{0} \leq u \leq u_{\mathfrak{N}}} \mathbb{D}\left(E_{u}^{+} u\right) \frac{\mathbb{M}_{u} w\left(E_{u}^{+} u\right)}{W\left(E_{u}^{+} u\right)} \frac{V\left(E_{u}^{+} u\right) W(x)-V(x) W\left(E_{u}^{+} u\right)}{x-E_{u}^{+} u}+" .
\end{aligned}
$$

Since the third term on the right-hand side of the previous equation is clearly a polynomial in $x$, we will focus next on the second term. The last factor of this latter term can be written as

$$
\begin{aligned}
\mathbb{M}_{x} f(x)-\int_{u_{0} \leq u \leq u_{\mathfrak{N}}} \mathbb{D}\left(E_{u}^{+} u\right) \frac{\mathbb{M}_{u} w\left(E_{u}^{+} u\right)}{x-E_{u}^{+} u} \\
=\int_{u_{0} \leq u \leq u_{\mathfrak{N}}} \mathbb{D} u w(u) \frac{1}{2}\left\{\frac{1}{E_{x}^{+} x-u}+\frac{1}{E_{x}^{-} x-u}\right\} \\
\quad-\frac{1}{2} \int_{u_{0} \leq u \leq u_{\mathfrak{N}}} \mathbb{D}\left(E_{u}^{+} u\right) \frac{w\left(E_{u}^{+2} u\right)}{x-E_{u}^{+} u}-\frac{1}{2} \int_{u_{0} \leq u \leq u_{\mathfrak{N}}} \mathbb{D}\left(E_{u}^{+} u\right) \frac{w(u)}{x-E_{u}^{+} u} .
\end{aligned}
$$

The last two terms of the above equation are

$$
\begin{aligned}
\int_{u_{0} \leq u \leq u_{\mathfrak{N}}} \mathbb{D}\left(E_{u}^{+} u\right) \frac{w\left(E_{u}^{+2} u\right)}{x-E_{u}^{+} u}+\int_{u_{0} \leq u \leq u_{\mathfrak{N}}} \mathbb{D}\left(E_{u}^{+} u\right) \frac{w(u)}{x-E_{u}^{+} u} \\
=\sum_{0 \leq s \leq \mathfrak{N}}\left[u_{s}-E_{u}^{-2} u_{s}\right] \frac{w\left(u_{s}\right)}{x-E_{u}^{-} u_{s}}+\sum_{0 \leq s \leq \mathfrak{N}}\left[E_{u}^{+2} u_{s}-u_{s}\right] \frac{w\left(u_{s}\right)}{x-E_{u}^{+} u_{s}} \\
\quad+\Delta y\left(E_{u}^{+} u_{\mathfrak{N}}\right) \frac{w\left(E_{u}^{+2} u_{\mathfrak{N}}\right)}{x-E_{u}^{+} u_{\mathfrak{N}}}-\Delta y\left(E_{u}^{-} u_{0}\right) \frac{w\left(u_{0}\right)}{x-E_{u}^{-} u_{0}} \\
=\sum_{0 \leq s \leq \mathfrak{N}} w\left(u_{s}\right)\left\{\frac{E_{u}^{+2} u_{s}-u_{s}}{x-E_{u}^{+} u_{s}}+\frac{u_{s}-E_{u}^{-2} u_{s}}{x-E_{u}^{-} u_{s}}\right\}+",
\end{aligned}
$$


where we have used the identity for integrals under the reparameterization of the lattice $u \mapsto E_{u}^{-2} u$ and introducing the additional boundary terms. Combining these results, we have

$$
\begin{aligned}
& \mathbb{M}_{x} f(x)-\int_{u_{0} \leq u \leq u_{\mathfrak{N}}} \mathbb{D}\left(E_{u}^{+} u\right) \frac{\mathbb{M}_{u} w\left(E_{u}^{+} u\right)}{x-E_{u}^{+} u} \\
&=\frac{1}{2} \sum_{0 \leq s \leq \mathfrak{N}} w\left(u_{s}\right)\left\{\left(E_{u}^{+} u_{s}-E_{u}^{-} u_{s}\right)\left(\frac{1}{E_{x}^{+} x-u_{s}}+\frac{1}{E_{x}^{-} x-u_{s}}\right)\right. \\
&\left.-\frac{E_{u}^{+2} u_{s}-u_{s}}{x-E_{u}^{+} u_{s}}-\frac{u_{s}-E_{u}^{-2} u_{s}}{x-E_{u}^{-} u_{s}}\right\} \\
&-\frac{1}{2} \Delta y\left(E_{u}^{+} u_{\mathfrak{N}}\right) \frac{w\left(E_{u}^{+2} u_{\mathfrak{N}}\right)}{x-E_{u}^{+} u_{\mathfrak{N}}}+\frac{1}{2} \Delta y\left(E_{u}^{-} u_{0}\right) \frac{w\left(u_{0}\right)}{x-E_{u}^{-} u_{0}} \\
&= \frac{1}{2} \sum_{0 \leq s \leq \mathfrak{N}} w\left(u_{s}\right)\left\{\left(E_{u}^{+} u_{s}-E_{u}^{-} u_{s}\right) \frac{\left(E_{x}^{+}+E_{x}^{-}\right) x-2 u_{s}}{\left(x-E_{u}^{+} u_{s}\right)\left(x-E_{u}^{-} u_{s}\right)}\right. \\
&\left.-\frac{E_{u}^{+2} u_{s}-u_{s}}{x-E_{u}^{+} u_{s}}-\frac{u_{s}-E_{u}^{-2} u_{s}}{x-E_{u}^{-} u_{s}}\right\}+",
\end{aligned}
$$

where we have used the ratio identity again. We observe that the summand appears to have two sets of simple poles at $x=E^{ \pm} u_{s}$, however when we compute their residues they vanish identically, implying that the summand is in reality a polynomial in $x$. This leaves us with the two sets of boundary terms to account for. We find that their combination is

$$
\begin{aligned}
& -\frac{w\left(E_{u}^{+2} u_{\mathfrak{N}}\right)}{x-E_{u}^{+} u_{\mathfrak{N}}}\left[W(x)-\Delta y\left(E_{u}^{+} u_{\mathfrak{N}}\right) V(x)\right] \\
& +\frac{w\left(u_{0}\right)}{x-E_{u}^{-} u_{0}}\left[W(x)-\Delta y\left(E_{u}^{-} u_{0}\right) V(x)\right] .
\end{aligned}
$$

The first term vanishes under the upper terminating condition, while the second is a polynomial in $x$ due to the lower terminating condition. In summary, this leads us to conclude that $W(x) \mathbb{D}_{x} f(x)-2 V(x) \mathbb{M}_{x} f(x)$ is a polynomial in $x$.

We offer the following analogue to the concept of a regular semiclassical weight.

Definition 4.2. A generic or regular $\mathbb{D}$-semiclassical weight has two properties: 
(i) strict inequalities in the degrees of the spectral data polynomials, that is, $\operatorname{deg}_{x} W=M, \operatorname{deg}_{x} V=M-1$, and $\operatorname{deg}_{x} U=M-2$, and

(ii) the lattice generated by any zero of $\left(W^{2}-\Delta y^{2} V^{2}\right)(x)$ does not coincide with the lattice generated by another zero; that is, for any $\tilde{x}$ such that $\left(W^{2}-\Delta y^{2} V^{2}\right)(\tilde{x})=0$, then $\left(W^{2}-\Delta y^{2} V^{2}\right)\left(E_{x}^{2 \mathbb{Z}} \tilde{x}\right) \neq 0$.

Conversely, any weight with data not satisfying these conditions will be termed an irregular $\mathbb{D}$-semiclassical weight. Note that the zeros of $W$ are not relevant and that no interpretation as a singularity can be placed upon a zero of $\left(W^{2}-\Delta y^{2} V^{2}\right)(x)$.

Proposition 4.2 ([62, (12)], [63, Theorem 1]). The spectral coefficients $W_{n}(x), \Theta_{n}(x)$, and $\Omega_{n}(x)$ are defined in terms of bilinear formulas of the polynomials and associated functions

$$
\begin{aligned}
& \frac{2}{a_{n}}\left(2 W_{n}(x)-W(x)\right) \\
& =(W+\Delta y V)\left[p_{n}\left(y_{+}\right) q_{n-1}\left(y_{-}\right)-p_{n-1}\left(y_{+}\right) q_{n}\left(y_{-}\right)\right] \\
& +(W-\Delta y V)\left[p_{n}\left(y_{-}\right) q_{n-1}\left(y_{+}\right)-p_{n-1}\left(y_{-}\right) q_{n}\left(y_{+}\right)\right], \quad n \geq 0, \\
& \Delta y \Theta_{n}(x) \\
& =(W+\Delta y V) p_{n}\left(y_{+}\right) q_{n}\left(y_{-}\right) \\
& -(W-\Delta y V) p_{n}\left(y_{-}\right) q_{n}\left(y_{+}\right), \quad n \geq 0, \\
& \frac{2 \Delta y}{a_{n}}\left(\Omega_{n}(x)+V(x)\right) \\
& =(W+\Delta y V)\left[p_{n}\left(y_{+}\right) q_{n-1}\left(y_{-}\right)+p_{n-1}\left(y_{+}\right) q_{n}\left(y_{-}\right)\right] \\
& +(W-\Delta y V)\left[-p_{n}\left(y_{-}\right) q_{n-1}\left(y_{+}\right)-p_{n-1}\left(y_{-}\right) q_{n}\left(y_{+}\right)\right], \quad n \geq 0 .
\end{aligned}
$$

For the $\mathbb{D}$-semiclassical class of weights, these coefficients are polynomials in $x$.

Proof. Our proof will be an extension of Laguerre's reasoning for the differential case. However, we first need to appreciate one important fact. Any polynomial in $y_{-}$and $y_{+}$is an element of the ring $\mathbb{C}[x]+\Delta y \mathbb{C}[x]$ because $y_{ \pm}=\mathbb{M}_{x} x \pm \frac{1}{2} \Delta y$, and $\mathbb{M}_{x} x$ is a linear polynomial in $x$ and $\Delta y^{2}$ is a quadratic polynomial in $x$. The starting point will be the definition for $q_{n}$, but inverted to solve for the Stieltjes function

$$
f=\frac{q_{n}+p_{n-1}^{(1)}}{p_{n}}
$$


for arbitrary $n \geq 0$. Now consider the formulation of (4.13) using this expression

$$
\begin{aligned}
0= & (W-\Delta y V) f\left(y_{+}\right)-(W+\Delta y V) f\left(y_{-}\right)-\Delta y U \\
= & \frac{(W-\Delta y V) q_{n}\left(y_{+}\right) p_{n}\left(y_{-}\right)-(W+\Delta y V) q_{n}\left(y_{-}\right) p_{n}\left(y_{+}\right)}{p_{n}\left(y_{+}\right) p_{n}\left(y_{-}\right)} \\
& +\frac{(W-\Delta y V) p_{n-1}^{(1)}\left(y_{+}\right) p_{n}\left(y_{-}\right)-(W+\Delta y V) p_{n-1}^{(1)}\left(y_{-}\right) p_{n}\left(y_{+}\right)}{p_{n}\left(y_{+}\right) p_{n}\left(y_{-}\right)} \\
& -\frac{\Delta y U p_{n}\left(y_{+}\right) p_{n}\left(y_{-}\right)}{p_{n}\left(y_{+}\right) p_{n}\left(y_{-}\right)} .
\end{aligned}
$$

So now we have $(W-\Delta y V) p_{n-1}^{(1)}\left(y_{+}\right) p_{n}\left(y_{-}\right)-(W+\Delta y V) p_{n-1}^{(1)}\left(y_{-}\right) p_{n}\left(y_{+}\right)$ $-\Delta y U p_{n}\left(y_{+}\right) p_{n}\left(y_{-}\right) \in \mathbb{C}[x]+\Delta y \mathbb{C}[x]$, and furthermore, because it is odd under $y_{+} \leftrightarrow y_{-}$, this quantity is actually an element of $\Delta y \mathbb{C}[x]$ only. Consequently $(W-\Delta y V) q_{n}\left(y_{+}\right) p_{n}\left(y_{-}\right)-(W+\Delta y V) q_{n}\left(y_{-}\right) p_{n}\left(y_{+}\right) \in \Delta y \mathbb{C}[x]$, and we define the polynomial $\Theta_{n}(x)$ by

$$
\Delta y \Theta_{n}(x)=(W+\Delta y V) q_{n}\left(y_{-}\right) p_{n}\left(y_{+}\right)-(W-\Delta y V) q_{n}\left(y_{+}\right) p_{n}\left(y_{-}\right)
$$

which is (4.15). To find the other relations, we employ the alternative form of $\Theta_{n}(x)$

$$
\begin{aligned}
\Delta y \Theta_{n}= & (W-\Delta y V) p_{n-1}^{(1)}\left(y_{+}\right) p_{n}\left(y_{-}\right) \\
& -(W+\Delta y V) p_{n-1}^{(1)}\left(y_{-}\right) p_{n}\left(y_{+}\right)-\Delta y U p_{n}\left(y_{+}\right) p_{n}\left(y_{-}\right),
\end{aligned}
$$

and multiply the left-hand side of (4.19) by the Casoratians $1=a_{n}\left[p_{n-1}\left(y_{ \pm}\right) \times\right.$ $\left.p_{n-1}^{(1)}\left(y_{ \pm}\right)-p_{n}\left(y_{ \pm}\right) p_{n-2}^{(1)}\left(y_{ \pm}\right)\right]$so that upon reorganizing we have

$$
\begin{gathered}
p_{n}\left(y_{+}\right)\left[(W+\Delta y V) p_{n-1}^{(1)}\left(y_{-}\right)+\Delta y U p_{n}\left(y_{-}\right)-\Delta y a_{n} \Theta_{n} p_{n-2}^{(1)}\left(y_{+}\right)\right] \\
=p_{n-1}^{(1)}\left(y_{+}\right)\left[(W-\Delta y V) p_{n}\left(y_{-}\right)-\Delta y a_{n} \Theta_{n} p_{n-1}\left(y_{+}\right)\right]
\end{gathered}
$$

and

$$
\begin{aligned}
p_{n}\left(y_{-}\right)\left[(W-\Delta y V) p_{n-1}^{(1)}\left(y_{+}\right)-\Delta y U p_{n}\left(y_{+}\right)+\Delta y a_{n} \Theta_{n} p_{n-2}^{(1)}\left(y_{-}\right)\right] \\
=p_{n-1}^{(1)}\left(y_{-}\right)\left[(W+\Delta y V) p_{n}\left(y_{+}\right)+\Delta y a_{n} \Theta_{n} p_{n-1}\left(y_{-}\right)\right] .
\end{aligned}
$$


Relation (4.20) implies that there must exist polynomials $\pi_{1}, \pi_{2} \in \mathbb{C}[x]$ such that

$$
\begin{aligned}
p_{n}\left(y_{+}\right) p_{n-1}^{(1)}\left(y_{+}\right)\left(\pi_{1}+\Delta y \pi_{2}\right) \\
=p_{n}\left(y_{+}\right)\left[(W+\Delta y V) p_{n-1}^{(1)}\left(y_{-}\right)\right. \\
\left.\quad+\Delta y U p_{n}\left(y_{-}\right)-\Delta y a_{n} \Theta_{n} p_{n-2}^{(1)}\left(y_{+}\right)\right] \\
\quad=p_{n-1}^{(1)}\left(y_{+}\right)\left[(W-\Delta y V) p_{n}\left(y_{-}\right)-\Delta y a_{n} \Theta_{n} p_{n-1}\left(y_{+}\right)\right]
\end{aligned}
$$

while (4.21) implies an identical relation with $y_{+} \leftrightarrow y_{-}$and $\Delta y \mapsto-\Delta y$. Therefore we have the pair of relations

$$
\begin{aligned}
& p_{n-1}^{(1)}\left(y_{+}\right)\left(\pi_{1}+\Delta y \pi_{2}\right) \\
& \quad=(W+\Delta y V) p_{n-1}^{(1)}\left(y_{-}\right)+\Delta y U p_{n}\left(y_{-}\right)-\Delta y a_{n} \Theta_{n} p_{n-2}^{(1)}\left(y_{+}\right) \\
& p_{n}\left(y_{+}\right)\left(\pi_{1}+\Delta y \pi_{2}\right) \\
& \quad=(W-\Delta y V) p_{n}\left(y_{-}\right)-\Delta y a_{n} \Theta_{n} p_{n-1}\left(y_{+}\right)
\end{aligned}
$$

and an analogous pair with $y_{+} \leftrightarrow y_{-}$. We form the combination of $p_{n-1}\left(y_{+}\right) \times$ $(4.24)-p_{n-2}^{(1)}\left(y_{+}\right) \times(4.25)$, and by employing the Casoratian and eliminating the associated polynomials we arrive at the solution

$$
\begin{aligned}
\pi_{1}+\Delta y \pi_{2}= & -a_{n}(W+\Delta y V) p_{n-1}\left(y_{+}\right) q_{n}\left(y_{-}\right) \\
& +a_{n}(W-\Delta y V) p_{n}\left(y_{-}\right) q_{n-1}\left(y_{+}\right) .
\end{aligned}
$$

A similar procedure on the other pair yields the partner relation

$$
\begin{aligned}
\pi_{1}-\Delta y \pi_{2}= & a_{n}(W+\Delta y V) p_{n}\left(y_{+}\right) q_{n-1}\left(y_{-}\right) \\
& -a_{n}(W-\Delta y V) p_{n-1}\left(y_{-}\right) q_{n}\left(y_{+}\right)
\end{aligned}
$$

The sum and difference of these are simply (4.14) and (4.16) with the identifications $\pi_{1}=2 W_{n}-W$ and $\pi_{2}=-\Omega_{n}-V$.

Knowledge of the large $x$ expansions of the spectral coefficients will be important in the ensuing analysis.

Proposition 4.3 ([62, (14), (22)]). The spectral coefficients have terminating expansions about $x=\infty$ with leading order terms 


$$
\begin{aligned}
W_{n}(x)= & \frac{1}{2} W+\frac{1}{4}[W+\Delta y V]\left(\frac{y_{+}}{y_{-}}\right)^{n} \\
& +\frac{1}{4}[W-\Delta y V]\left(\frac{y_{-}}{y_{+}}\right)^{n}+\mathrm{O}\left(x^{M-1}\right), \quad n \geq 0, \\
\Theta_{n}(x)= & \frac{1}{y_{-} \Delta y}[W+\Delta y V]\left(\frac{y_{+}}{y_{-}}\right)^{n} \\
& -\frac{1}{y_{+} \Delta y}[W-\Delta y V]\left(\frac{y_{-}}{y_{+}}\right)^{n}+\mathrm{O}\left(x^{M-3}\right), \quad n \geq 0, \\
\Omega_{n}(x)+V(x)= & \frac{1}{2 \Delta y}[W+\Delta y V]\left(\frac{y_{+}}{y_{-}}\right)^{n}{ }^{n}, \quad n \geq 0 . \\
& -\frac{1}{2 \Delta y}[W-\Delta y V]\left(\frac{y_{-}}{y_{+}}\right)^{n}+\mathrm{O}\left(x^{M-2}\right), \quad n \geq 0
\end{aligned}
$$

Proof. These expansions follow from the substitution of the expansions for the polynomials (3.34) and associated functions (3.35) into the formulas (4.14)-(4.16). One should note that $y_{+} / y_{-}=\mathrm{O}(1)$ as $x \rightarrow \infty$.

Corollary 4.2 ([62, p. 220], [63, Theorem 1]). In the generic or regular $\mathbb{D}$-semiclassical case $W_{n}, \Theta_{n}, \Omega_{n}$ are polynomials in $x$ with fixed degrees independent of $n$, specifically $\operatorname{deg}_{x} W_{n}=M$, $\operatorname{deg}_{x} \Omega_{n}=M-1$ and $\operatorname{deg}_{x} \Theta_{n}=$ M-2.

Proof. This follows from the Proposition 4.3 after recalling that $y_{ \pm}=$ $\mathrm{O}(x)$ as $x \rightarrow \infty$.

The expressions for the spectral coefficients (4.14)-(4.16) can be inverted yielding a system of coupled first-order linear divided-difference equations for the polynomials and associated functions with respect to $x$.

Proposition 4.4 ([63, Theorem 1], [62, p. 221]). The polynomials with a $\mathbb{D}$-semiclassical weight satisfy

$$
W_{n} \mathbb{D}_{x} p_{n}=\Omega_{n} \mathbb{M}_{x} p_{n}-a_{n} \Theta_{n} \mathbb{M}_{x} p_{n-1}, \quad n \geq 0,
$$

for certain spectral coefficients, $W_{n}(x), \Theta_{n}(x), \Omega_{n}(x)$. The partner equation to (4.31) is

$$
W_{n} \mathbb{D}_{x} p_{n-1}=-\left(\Omega_{n}+2 V\right) \mathbb{M}_{x} p_{n-1}+a_{n} \Theta_{n-1} \mathbb{M}_{x} p_{n}, \quad n \geq 0 .
$$

The associated functions satisfy divided-difference equations

$$
W_{n} \mathbb{D}_{x} q_{n}=\left(\Omega_{n}+2 V\right) \mathbb{M}_{x} q_{n}-a_{n} \Theta_{n} \mathbb{M}_{x} q_{n-1}, \quad n \geq 0,
$$




$$
W_{n} \mathbb{D}_{x} q_{n-1}=-\Omega_{n} \mathbb{M}_{x} q_{n-1}+a_{n} \Theta_{n-1} \mathbb{M}_{x} q_{n}, \quad n \geq 0,
$$

identical in structure to (4.31) and (4.32), respectively. The coupled system in (4.31), (4.32), (4.33), and (4.34) can be written in matrix form as the spectral divided-difference equation

$$
\begin{aligned}
\mathbb{D}_{x} & Y_{n}(x) \\
& :=A_{n} \mathbb{M}_{x} Y_{n}(x) \\
& =\frac{1}{W_{n}(x)}\left(\begin{array}{cc}
\Omega_{n}(x) & -a_{n} \Theta_{n}(x) \\
a_{n} \Theta_{n-1}(x) & -\Omega_{n}(x)-2 V(x)
\end{array}\right) \mathbb{M}_{x} Y_{n}(x), \quad n \geq 0,
\end{aligned}
$$

with $A_{n}$ termed the spectral matrix.

Proof. Our derivation of the spectral divided-difference equations will employ a simple method - that of inversion of the relations for the spectral coefficients (4.14)-(4.16). Consider the following equivalent forms of those relations

$$
\begin{aligned}
2 W_{n} & -W+\Delta y\left(\Omega_{n}+V\right) \\
= & a_{n}\left[(W+\Delta y V) p_{n}\left(y_{+}\right) q_{n-1}\left(y_{-}\right)\right. \\
& \left.-(W-\Delta y V) p_{n-1}\left(y_{-}\right) q_{n}\left(y_{+}\right)\right], \\
2 W_{n} & -W-\Delta y\left(\Omega_{n}+V\right) \\
= & a_{n}\left[-(W+\Delta y V) p_{n-1}\left(y_{+}\right) q_{n}\left(y_{-}\right)\right. \\
& \left.\quad+(W-\Delta y V) p_{n}\left(y_{-}\right) q_{n-1}\left(y_{+}\right)\right], \\
\Delta y \Theta_{n} & \\
= & (W+\Delta y V) p_{n}\left(y_{+}\right) q_{n}\left(y_{-}\right)-(W-\Delta y V) p_{n}\left(y_{-}\right) q_{n}\left(y_{+}\right) .
\end{aligned}
$$

For our first task, that of deriving (4.31), we construct the combination $p_{n}\left(y_{+}\right) \times(4.37)-p_{n}\left(y_{-}\right) \times(4.36)+\left(p_{n-1}\left(y_{+}\right)+p_{n-1}\left(y_{-}\right)\right) a_{n} \times(4.38)$. After effecting some initial cancellation we can then employ the Casoratian relation (3.26) to make further simplification, and our final result is precisely (4.31) in finite-difference form. The approach taken for the other relations (4.32)-(4.34) is the same. For (4.32) we use the combination $p_{n-1}\left(y_{+}\right) \times$ $(4.36)-p_{n-1}\left(y_{-}\right) \times(4.37)-\left(p_{n}\left(y_{+}\right)+p_{n}\left(y_{-}\right)\right) a_{n} \times\left.(4.38)\right|_{n \mapsto n-1}$, while for (4.33) we use $q_{n}\left(y_{+}\right) \times(4.37)-q_{n}\left(y_{-}\right) \times(4.36)+\left(q_{n-1}\left(y_{+}\right)+q_{n-1}\left(y_{-}\right)\right) a_{n} \times$ (4.38), and for $(4.34)$ we use $q_{n-1}\left(y_{+}\right) \times(4.36)-q_{n-1}\left(y_{-}\right) \times(4.37)-$ $\left(q_{n}\left(y_{+}\right)+q_{n}\left(y_{-}\right)\right) a_{n} \times\left.(4.38)\right|_{n \mapsto n-1}$. 
The initial values of the spectral coefficients are specified by the weight data $W, V, U$ and the initial norm $\gamma_{0}$ :

$$
\begin{aligned}
& W_{-1}=0, W_{0}=W, \quad \Theta_{-1}=0, \quad \Theta_{0}=-\gamma_{0}^{2} U \\
& \Omega_{0}=0, \quad \Omega_{1}=-2 V-\gamma_{0}^{2}\left(\mathbb{M}_{x} x-b_{0}\right) U .
\end{aligned}
$$

REMARK 4.4. An alternative line of reasoning to achieve the above results - and in fact to generalize them beyond the $\mathbb{D}$-semiclassical class - is the following. By positing an explicit quadrature formula for the nonlinear lattice, one upon which the orthogonality condition is founded, one can derive (4.31), (4.32), and (4.35), assuming the existence of certain moments of "log-derivative" of the weight $\mathbb{D}_{x} w / \mathbb{M}_{x} w$. A by-product of this approach is that one also constructs an explicit quadrature formula for the spectral coefficients, albeit one which involves the polynomials themselves.

The spectral structure in Proposition 4.4 has been derived before for OPS with respect to general classes of weights on the linear lattice by [29], [67], [44], and [68], and on the q-linear lattice by [55], [67], [42], [54], [20], [32], [46], [70], and [79]. This has also been done for specific weights on the $q$-linear lattice in [72] and [15], and for OPS on the unit circle subject to $q$ difference relations in [48] and [14]. In addition to the studies by Magnus, the works [9] and [28] have investigated the spectral structures for general SNUL and arbitrary $\operatorname{deg}_{x} W, \operatorname{deg}_{x} V$. Characterization theorems for the classical OPS of the Askey table have been made by [22], [1], and others using an equivalent formulation to the spectral structures on general SNUL, but of course restricted to the $\operatorname{deg}_{x} W=2, \operatorname{deg}_{x} V=1$ case.

The compatibility relation between the matrix recurrence relation and the spectral divided-difference equation imposes the following conditions. The Cayley transform $\left(1-\frac{1}{2} \Delta y A\right)^{-1}\left(1+\frac{1}{2} \Delta y A\right)$ will figure prominently in the ensuing analysis.

Proposition 4.5. The spectral matrix and the recurrence matrix satisfy

$$
\begin{aligned}
& A_{n+1} \cdot \mathbb{M}_{x} K_{n}-\mathbb{M}_{x} K_{n} \cdot A_{n} \\
& \quad=\mathbb{D}_{x} K_{n}-\frac{1}{4} \Delta y^{2} A_{n+1} \cdot \mathbb{D}_{x} K_{n} \cdot A_{n}, \quad n \geq 0,
\end{aligned}
$$


or equivalently

$$
\begin{aligned}
& K_{n}\left(y_{+}\right)\left(1-\frac{1}{2} \Delta y A_{n}\right)^{-1}\left(1+\frac{1}{2} \Delta y A_{n}\right) \\
& \quad=\left(1-\frac{1}{2} \Delta y A_{n+1}\right)^{-1}\left(1+\frac{1}{2} \Delta y A_{n+1}\right) K_{n}\left(y_{-}\right), \quad n \geq 0 .
\end{aligned}
$$

Proof. The finite-difference form of (4.35) is

$$
\left(1-\frac{1}{2} \Delta y A_{n}\right) Y_{n}\left(y_{+}\right)=\left(1+\frac{1}{2} \Delta y A_{n}\right) Y_{n}\left(y_{-}\right),
$$

which demonstrates why the Cayley transform arises. The second relation (4.41) is found by computing $Y_{n+1}\left(y_{+}\right)$in two different ways from $Y_{n}\left(y_{-}\right)$ corresponding to the different orders of the operations $n \mapsto n+1$ and $y_{-} \mapsto$ $y_{+}$. The first relation (4.40) is derived by computing $\mathbb{D}_{x} Y_{n+1}$ in terms of $\mathbb{M}_{x} Y_{n}$ in two different orders.

Compatibility of the three-term recurrence relation and the spectral divided-difference equation implies the following result.

Proposition 4.6 ([60, (3.2)], [62, (16), (19)]). The spectral coefficients arising in Proposition 4.4 satisfy recurrence relations in $n$,

$$
\begin{gathered}
W_{n+1}=W_{n}+\frac{1}{4} \Delta y^{2} \Theta_{n}, \quad n \geq 0, \\
\Omega_{n+1}+\Omega_{n}+2 V=\left(\mathbb{M}_{x} x-b_{n}\right) \Theta_{n}, \quad n \geq 0, \\
\left(W_{n} \Omega_{n+1}-W_{n+1} \Omega_{n}\right)\left(\mathbb{M}_{x} x-b_{n}\right) \\
=-\frac{1}{4} \Delta y^{2} \Omega_{n+1} \Omega_{n}+W_{n} W_{n+1}+a_{n+1}^{2} W_{n} \Theta_{n+1} \\
-a_{n}^{2} W_{n+1} \Theta_{n-1}, \quad n \geq 0 .
\end{gathered}
$$

Proof. We offer a proof in the spirit of our previous proofs. For the first relation (4.43), we construct $2 W_{n+1}-2 W_{n}-\frac{1}{2} \Delta y^{2} \Theta_{n}$ and substitute the expressions (4.14) and (4.15) for $W_{n}$ and $\Theta_{n}$, respectively. After collecting terms proportional to $W \pm \Delta y V$, we observe that the coefficients of these terms separate into two parts, in which the three-term recurrence relation can be employed. The result we find is that they identically vanish. The second relation (4.44) can be found in an identical manner starting with $\Delta y\left[\Omega_{n+1}+\Omega_{n}+2 V-\left(\mathbb{M}_{x} x-b_{n}\right) \Theta_{n}\right]$. 
The relations of Proposition 4.6 constitute the analogues of the LaguerreFreud equations and have been studied in the special case of linear lattices by [29], [65], [44], and [68], and in the $q$-linear case by [55], [54], [70], and [32]. For the situation of OPS on the unit circle with $q$-difference operators, some of these relations can be found in [48]. Following on from the Magnus studies, the works [9] and [28] have investigated these compatibility conditions for general SNUL. Together with Proposition 4.4 this result allows for the construction of ladder (or raising and lowering) operators and these have been found in the $q$-linear lattice by [20] and [46].

A consequence of Proposition 4.6 is the following.

Proposition 4.7 ([60, Remark 3.2]). The spectral coefficients satisfy the bilinear

recurrence relation

$$
\begin{aligned}
W_{n}\left(W_{n}-W\right) & =\frac{1}{4} \Delta y^{2}\left[\Omega_{n}\left(\Omega_{n}+2 V\right)-a_{n}^{2} \Theta_{n-1} \Theta_{n}\right] \\
& =-\frac{1}{4} \Delta y^{2} \operatorname{det}\left(\begin{array}{cc}
\Omega_{n} & -a_{n} \Theta_{n} \\
a_{n} \Theta_{n-1} & -\Omega_{n}-2 V
\end{array}\right), \quad n \geq 0
\end{aligned}
$$

Proof. Two methods of proof are available here. The first method starts from the observation that the above bilinear relation can be expressed as

$$
\begin{aligned}
& {\left[2 W_{n}-W+\Delta y\left(\Omega_{n}+V\right)\right]\left[2 W_{n}-W-\Delta y\left(\Omega_{n}+V\right)\right]} \\
& \quad+a_{n}^{2} \Delta y^{2} \Theta_{n} \Theta_{n-1} \\
& =W^{2}-\Delta y^{2} V^{2} .
\end{aligned}
$$

Now we have evaluations of the factors appearing in the left-hand side of the preceding equation from the proof of Proposition 4.2, namely, (4.26), (4.27), and (4.18), in terms of products of a polynomial and the associated function. Substituting these evaluations into the left-hand side, effecting considerable cancellation of terms, and employing the Casoratian relation (3.26), the final result simplifies to the right-hand side.

The second method uses the compatibility relations (4.43)-(4.45) and constructs an integral for this coupled system. First, one multiplies the left-hand side of (4.44) by the left-hand side of (4.45) and cancels out the common factor of $\mathbb{M} x-b_{n}$ in the resulting equation. By judicious use of (4.43), one can effect the cancellation of four terms in this equation and a 
recasting of the terms linear in $\Theta_{n}$. Consequently, we arrive at the following equation

$$
\begin{aligned}
& \frac{\left(\Omega_{n+1}+V\right)^{2}}{W_{n+1}}-\frac{\left(\Omega_{n}+V\right)^{2}}{W_{n}} \\
& =\frac{4}{\Delta y^{2}}\left(W_{n+1}-W_{n}\right)+V^{2}\left[\frac{1}{W_{n+1}}-\frac{1}{W_{n}}\right] \\
& \quad+a_{n+1}^{2} \frac{\Theta_{n+1} \Theta_{n}}{W_{n+1}}-a_{n}^{2} \frac{\Theta_{n} \Theta_{n-1}}{W_{n}}
\end{aligned}
$$

which is manifestly a perfect difference equation in $n$. By summing this and employing the initial value evaluations (4.39), we have (4.46).

The above proposition must be augmented with the following matrix identities.

Corollary 4.3. The matrix factor appearing in Proposition 4.5 has the determinant evaluation

$$
\operatorname{det}\left(1 \pm \frac{1}{2} \Delta y A_{n}\right)=\frac{W \mp \Delta y V}{W_{n}}, \quad n \geq 0,
$$

and its inverse is

$$
\begin{aligned}
(1 & \left. \pm \frac{1}{2} \Delta y A_{n}\right)^{-1} \\
& =\frac{1}{W \mp \Delta y V}\left(\begin{array}{cc}
W_{n} \mp \frac{1}{2} \Delta y\left(\Omega_{n}+2 V\right) & \pm \frac{1}{2} \Delta y a_{n} \Theta_{n} \\
\mp \frac{1}{2} \Delta y a_{n} \Theta_{n-1} & W_{n} \pm \frac{1}{2} \Delta y \Omega_{n}
\end{array}\right), \quad n \geq 0 .
\end{aligned}
$$

Proof. From the form for the spectral matrix given by (4.35), we have

$$
\begin{aligned}
& \operatorname{det}\left(1 \pm \frac{1}{2} \Delta y A_{n}\right) \\
& =\frac{1}{W_{n}^{2}}\left[\left(W_{n} \pm \frac{1}{2} \Delta y \Omega_{n}\right)\left(W_{n} \mp \frac{1}{2} \Delta y\left(\Omega_{n}+2 V\right)\right)+\frac{1}{4} \Delta y^{2} a_{n}^{2} \Theta_{n} \Theta_{n-1}\right] \\
& =\frac{1}{W_{n}^{2}}\left[W_{n}^{2} \mp \Delta y V W_{n}-\frac{1}{4} \Delta y^{2}\left[\Omega_{n}\left(\Omega_{n}+2 V\right)-a_{n}^{2} \Theta_{n} \Theta_{n-1}\right]\right] \\
& =\frac{W \mp \Delta y V}{W_{n}},
\end{aligned}
$$

where we have employed the previous corollary in the last step. Constructing the matrix inverse given on the left-hand side of (4.50) and using our determinant formula, we find (4.50). 
Consequent to the results of Corollary 4.3, we require the matrix product found in (4.41).

COROLlARY 4.4. The matrix product appearing in (4.41) has the evaluation

$$
\begin{aligned}
& \left(1-\frac{1}{2} \Delta y A_{n}\right)^{-1}\left(1+\frac{1}{2} \Delta y A_{n}\right) \\
& =\frac{1}{W+\Delta y V} \\
& \times\left(\begin{array}{cc}
2 W_{n}-W+\Delta y\left(\Omega_{n}+V\right) & -\Delta y a_{n} \Theta_{n} \\
\Delta y a_{n} \Theta_{n-1} & 2 W_{n}-W-\Delta y\left(\Omega_{n}+V\right)
\end{array}\right), \\
& n \geq 0 \text {, } \\
& =a_{n}\left(\begin{array}{c}
p_{n}\left(y_{+}\right) \\
p_{n-1}\left(y_{+}\right)
\end{array}\right) \otimes\left(q_{n-1}\left(y_{-}\right), \quad-q_{n}\left(y_{-}\right)\right) \\
& -a_{n} \frac{W-\Delta y V}{W+\Delta y V}\left(\begin{array}{c}
q_{n}\left(y_{+}\right) \\
q_{n-1}\left(y_{+}\right)
\end{array}\right) \otimes\left(p_{n-1}\left(y_{-}\right), \quad-p_{n}\left(y_{-}\right)\right) .
\end{aligned}
$$

Proof. Using (4.50), we multiply out the matrix product and employ the bilinear identity (4.46) to simplify the diagonal elements, resulting in (4.51).

This result motivates the following definitions:

$$
\begin{aligned}
\mathfrak{W}_{ \pm} & :=2 W_{n}-W \pm \Delta y\left(\Omega_{n}+V\right), \quad n \geq 1, \\
\mathfrak{T}_{+} & :=\Delta y a_{n} \Theta_{n}, \quad \mathfrak{T}_{-}:=\Delta y a_{n} \Theta_{n-1}, \quad n \geq 1,
\end{aligned}
$$

while for $n=0$, we have $\mathfrak{W}_{ \pm}(n=0):=W \pm \Delta y V, \mathfrak{T}_{+}(n=0):=-\Delta y a_{0} \gamma_{0}^{2} U$ $\mathfrak{T}_{-}(n=0):=0$ together with

$$
A_{n}^{*}:=\left(\begin{array}{cc}
\mathfrak{W}_{+} & -\mathfrak{T}_{+} \\
\mathfrak{T}_{-} & \mathfrak{W}_{-}
\end{array}\right) .
$$

The variables $\mathfrak{W}_{ \pm}$are essentially the variables $U_{n}, X_{n}$ employed by Magnus in [62], respectively, while the variables $\mathfrak{T}_{ \pm}$are directly related to his $Y_{n}, Z_{n}$ variables. In these new variables the bilinear relation (4.46) becomes

$$
\operatorname{det} A_{n}^{*}=\mathfrak{W}_{+} \mathfrak{W}_{-}+\mathfrak{T}_{+} \mathfrak{T}_{-}=W^{2}-\Delta y^{2} V^{2},
$$

which will be the more useful form. 
It is of advantage to write out recurrence-spectral compatibility equations in terms of $\mathfrak{W}_{ \pm, n}, \mathfrak{T}_{ \pm, n}$, where we append a subscript to indicate the dependence on the index $n$.

Corollary 4.5. Solving (4.41) for $A_{n+1}^{*}$, we deduce

$$
\begin{aligned}
a_{n} a_{n+1} \mathfrak{T}_{+, n+1}= & -a_{n}\left(y_{+}-b_{n}\right) \mathfrak{W}_{+, n}+a_{n}\left(y_{-}-b_{n}\right) \mathfrak{W}_{-, n} \\
& +\left(y_{+}-b_{n}\right)\left(y_{-}-b_{n}\right) \mathfrak{T}_{+, n}+a_{n}^{2} \mathfrak{T}_{-, n}, \\
a_{n} \mathfrak{T}_{-, n+1}= & a_{n+1} \mathfrak{T}_{+, n}, \\
a_{n} \mathfrak{W}_{+, n+1}= & a_{n} \mathfrak{W}_{-, n}+\left(y_{+}-b_{n}\right) \mathfrak{T}_{+, n}, \\
a_{n} \mathfrak{W}_{-, n+1}= & a_{n} \mathfrak{W}_{+, n}-\left(y_{-}-b_{n}\right) \mathfrak{T}_{+, n} .
\end{aligned}
$$

A significant result follows from the observation that the right-hand side of (4.55) is a polynomial in $x$ with fixed degree independent of $n$, and in fact contains no dependence on $n$. If we denote one of the zeros of the spectral polynomial $W^{2}-\Delta y^{2} V^{2}$ as $x_{j}$, then we can apply the equality for $n \mapsto n+1$ and use (4.57) to deduce

$$
\mathfrak{W}_{+, n+1}\left(x_{j}\right) \mathfrak{W}_{-, n+1}\left(x_{j}\right)+\frac{a_{n+1}}{a_{n}} \mathfrak{T}_{+, n+1}\left(x_{j}\right) \mathfrak{T}_{+, n}\left(x_{j}\right)=0 .
$$

This then allows us to draw the following conclusion.

Proposition 4.8. The variable $\mathfrak{T}_{+, n+1}\left(x_{j}\right)$, when written in terms of the variables at $n$, factorizes in the following way:

$$
\begin{aligned}
&-a_{n} a_{n+1} \mathfrak{T}_{+, n+1}\left(x_{j}\right) \mathfrak{T}_{+, n}\left(x_{j}\right) \\
&= {\left[a_{n} \mathfrak{W}_{-, n}\left(x_{j}\right)+\left(y_{+, j}-b_{n}\right) \mathfrak{T}_{+, n}\left(x_{j}\right)\right] } \\
& \quad \times\left[a_{n} \mathfrak{W}_{+, n}\left(x_{j}\right)-\left(y_{-, j}-b_{n}\right) \mathfrak{T}_{+, n}\left(x_{j}\right)\right] .
\end{aligned}
$$

The coupled pair of first-order divided-difference equations (4.31) and (4.32) imply a second-order equation for one of the components, say $p_{n}$.

Proposition 4.9 ([60, Theorem 6.1], [8]). The $\mathbb{D}$-semiclassical orthogonal polynomials or associated functions satisfy a second-order divideddifference equation in the following two equivalent forms: either 


$$
\begin{aligned}
& {\left[\mathbb{M}_{x}\left(\frac{W_{n}}{\Theta_{n}}\right)+\frac{1}{4} \Delta y^{2} \mathbb{D}_{x}\left(\frac{\Omega_{n}+2 V}{\Theta_{n}}\right)\right] \mathbb{D}_{x}^{2} p_{n}} \\
& \quad+\left[\mathbb{D}_{x}\left(\frac{W_{n}}{\Theta_{n}}\right)+\mathbb{M}_{x}\left(\frac{2 V}{\Theta_{n}}\right)+\Delta y^{2} \mathbb{M}_{x}\left(\frac{W-W_{n}}{\Delta y^{2} \Theta_{n}}\right)\right] \mathbb{M}_{x} \mathbb{D}_{x} p_{n} \\
& \quad+\left[\mathbb{M}_{x}\left(4 \frac{W-W_{n}}{\Delta y^{2} \Theta_{n}}\right)-\mathbb{D}_{x}\left(\frac{\Omega_{n}}{\Theta_{n}}\right)\right] \mathbb{M}_{x}^{2} p_{n}=0
\end{aligned}
$$

or alternatively

$$
\begin{aligned}
& E_{x}^{+}\left(\frac{W+\Delta y V}{\Delta y \Theta_{n}}\right)\left(E_{x}^{+}\right)^{2} p_{n}+E_{x}^{-}\left(\frac{W-\Delta y V}{\Delta y \Theta_{n}}\right)\left(E_{x}^{-}\right)^{2} p_{n} \\
& \quad+\left\{-E_{x}^{+}\left(\frac{2 W_{n}-W+\Delta y\left(\Omega_{n}+V\right)}{\Delta y \Theta_{n}}\right)\right. \\
& \left.\quad-E_{x}^{-}\left(\frac{2 W_{n}-W-\Delta y\left(\Omega_{n}+V\right)}{\Delta y \Theta_{n}}\right)\right\} E_{x}^{+} E_{x}^{-} p_{n}=0 .
\end{aligned}
$$

Proof. Starting with (4.31) and (4.32), we can employ (4.46) to write an equation for each of $\mathbb{M}_{x} p_{n-1}$ and $\mathbb{D}_{x} p_{n-1}$ in terms of linear combinations of $\mathbb{M}_{x} p_{n}$ and $\mathbb{D}_{x} p_{n}$. Then utilizing the fact that $\mathbb{M}_{x} \mathbb{D}_{x} p_{n-1}=\mathbb{D}_{x} \mathbb{M}_{x} p_{n-1}$, we arrive at (4.62). The equation (4.63) is the nodal equivalent of the former.

This concludes our discussion of the spectral structures for a general quadratic lattice, and we now turn to the simplest explicit example on the $q$-quadratic lattice.

\section{$\S 5$. The $M=2, L=0$ cases and the Askey-Wilson polynomials}

Here we will employ the theory of Section 4 and explicitly compute the spectral coefficients for the Askey-Wilson system itself because it serves as an instructive example for the theory of the previous section and clarifies some confusion present in the literature. Virtually all of our results presented here have been found by earlier studies: first, by Askey and Wilson [4], then most notably by the Soviet school of Nikiforov, Suslov, and Uvarov [76], [73], [77], [74], [75] who were primarily concerned with hypergeometric type OPS on nonuniform lattices, and in the 1988 work of Magnus [60], whose approach and intent are most similar to the spirit of our own. 
We recall that the Askey-Wilson [4] weight itself has degrees $2 N=4$ or $M=2$ and $L=0$ with

$$
\begin{aligned}
w(x) & =w\left(x ;\left\{a_{1}, \ldots, a_{4}\right\}\right)=\frac{\left(z^{ \pm 2} ; q\right)_{\infty}}{\sin (\theta) \prod_{j=1}^{4}\left(a_{j} z^{ \pm 1} ; q\right)_{\infty}}, \\
G & =(-1,1) .
\end{aligned}
$$

Let the $j$ th elementary symmetric polynomial of $a_{1}, \ldots, a_{4}$ be denoted by $\sigma_{j}$ for $j=0, \ldots, 4$.

Proposition 5.1. Assume that $q \neq 1$ and $\left|q^{-1 / 2} a_{j}\right| \neq 1$ for $j=1, \ldots, 4$. The spectral coefficients for the Askey-Wilson OPS are

$$
\begin{aligned}
W_{n}(x)= & \left(1+q^{-n}\right)\left(1+\sigma_{4} q^{n-2}\right)\left(x^{2}-1\right) \\
& -\left[q^{-1 / 2} \sigma_{1}+q^{-3 / 2} \sigma_{3}\right] x+1+q^{-1} \sigma_{2}+q^{-2} \sigma_{4}, \\
\Omega_{n}(x)+V(x)= & 2 \frac{q^{n-2} \sigma_{4}-q^{-n}}{q^{1 / 2}-q^{-1 / 2}} x \\
& +\frac{q^{-n-1 / 2} \sigma_{1}+\left(-2+q^{-n}\right) q^{-3 / 2} \sigma_{3}}{\left(q^{1 / 2}-q^{-1 / 2}\right)\left(q^{-n}-q^{n-2} \sigma_{4}\right)} \\
& +\frac{\left(-2+q^{n}\right) q^{-5 / 2} \sigma_{1} \sigma_{4}+q^{n-7 / 2} \sigma_{3} \sigma_{4}}{\left(q^{1 / 2}-q^{-1 / 2}\right)\left(q^{-n}-q^{n-2} \sigma_{4}\right)} \\
\Theta_{n}(x)= & 4 \frac{q^{n-3 / 2} \sigma_{4}-q^{-n-1 / 2}}{q^{1 / 2}-q^{-1 / 2}}
\end{aligned}
$$

valid for $n \geq 0$. The three-term recurrence coefficients are found to be given by the standard expressions (see [56], [58])

$$
a_{n}^{2}=\frac{1}{4} \frac{\left(1-q^{n}\right)\left(1-\sigma_{4} q^{n-2}\right) \prod_{k>j}\left(1-a_{j} a_{k} q^{n-1}\right)}{\left(1-\sigma_{4} q^{2 n-3}\right)\left(1-\sigma_{4} q^{2 n-2}\right)^{2}\left(1-\sigma_{4} q^{2 n-1}\right)}
$$

and

$$
\begin{aligned}
b_{n}= & {\left[\sigma_{1}\left(q+\sigma_{4}\left(q^{2 n}-q^{n}-q^{n-1}\right)\right)+\sigma_{3}\left(1-q^{n}-q^{n+1}+\sigma_{4} q^{2 n-1}\right)\right] } \\
& \times \frac{q^{n-1}}{2\left(1-\sigma_{4} q^{2 n}\right)\left(1-\sigma_{4} q^{2 n-2}\right)},
\end{aligned}
$$

for $n \geq 0$, where we assume that $a_{0}=0$. 
Proof. From the weight (5.1), we compute the spectral data

$$
W \pm \Delta y V=z^{\mp 2} \prod_{j=1}^{4}\left(1-a_{j} q^{-1 / 2} z^{ \pm 1}\right),
$$

from which we deduce

$$
\begin{aligned}
& W(x)=2\left(1+\sigma_{4} q^{-2}\right) x^{2}-\left[q^{-1 / 2} \sigma_{1}+q^{-3 / 2} \sigma_{3}\right] x-1+q^{-1} \sigma_{2}-q^{-2} \sigma_{4} \\
& V(x)=2 \frac{q^{-2} \sigma_{4}-1}{q^{1 / 2}-q^{-1 / 2}} x+\frac{q^{-1 / 2} \sigma_{1}-q^{-3 / 2} \sigma_{3}}{q^{1 / 2}-q^{-1 / 2}}
\end{aligned}
$$

We will see that $W^{2}-\Delta y^{2} V^{2}$ will play a significant role and therefore define another set of elementary symmetric polynomials by

$$
W^{2}-\Delta y^{2} V^{2}=K^{2}\left[x^{4}-e_{1} x^{3}+e_{2} x^{2}-e_{3} x+e_{4}\right] .
$$

We note the evaluation $K=4 q^{\mu}=4 q^{-1} \sqrt{\sigma_{4}}$. We parameterize the spectral coefficients in the following way:

$$
\begin{aligned}
2 W_{n}-W & =w_{2} x^{2}+w_{1} x+w_{0}, \\
\Theta_{n} & =\varpi_{+}, \quad \Theta_{n-1}=\varpi_{-}, \\
\Omega_{n}+V & =v_{1} x+v_{0},
\end{aligned}
$$

where we know from (4.28)-(4.30) that the leading coefficients are

$$
\begin{aligned}
& w_{2}=\frac{K}{2}\left(q^{n+\mu}+q^{-n-\mu}\right), \quad v_{1}=\frac{K}{2} \frac{q^{n+\mu}-q^{-n-\mu}}{q^{1 / 2}-q^{-1 / 2}}, \\
& \varpi_{+}=K \frac{q^{n+\mu+1 / 2}-q^{-n-\mu-1 / 2}}{q^{1 / 2}-q^{-1 / 2}} .
\end{aligned}
$$

From the fundamental bilinear relation (4.46), we get a system of quadratic polynomial equalities

$$
\begin{aligned}
w_{2}^{2}-\Delta v_{1}^{2} & =K^{2}, \\
2 w_{1} w_{2}-2 \Delta v_{0} v_{1} & =-K^{2} e_{1}, \\
2 w_{0} w_{2}+w_{1}^{2}-\Delta\left(v_{0}^{2}-v_{1}^{2}\right)+a_{n}^{2} \Delta \varpi_{+} \varpi_{-} & =K^{2} e_{2}, \\
2 w_{0} w_{1}+2 \Delta v_{0} v_{1} & =-K^{2} e_{3},
\end{aligned}
$$




$$
w_{0}^{2}+\Delta v_{0}^{2}-a_{n}^{2} \Delta \varpi_{+} \varpi_{-}=K^{2} e_{4},
$$

where $\Delta:=\left(q^{1 / 2}-q^{-1 / 2}\right)^{2}$. Now (5.15), (5.17), and (5.19) imply $w_{1}^{2}+$ $\left(w_{0}+w_{2}\right)^{2}=K^{2}\left(1+e_{2}+e_{4}\right)$, while (5.18) and (5.16) imply $2 w_{1}\left(w_{0}+w_{2}\right)=$ $-K^{2}\left(e_{1}+e_{3}\right)$. Forming the sum and difference of these two latter relations, we conclude that

$$
w_{1}=\frac{W(1)-W(-1)}{2}, \quad w_{0}=-w_{2}+\frac{W(1)+W(-1)}{2} .
$$

Using (5.16), for example, along with the above solutions, we find that

$$
v_{0}=\frac{K^{2} e_{1}+w_{2}(W(1)-W(-1))}{2 \Delta v_{1}} .
$$

Therefore, we have succeeded in relating the subleading coefficients in terms of explicitly known quantities, and after simplification we arrive at (5.2)(5.3). Finally, using (5.19), we can solve for $a_{n}^{2}$ and, after observing that $e_{4}$ can be expressed as

$$
\begin{aligned}
e_{4}= & {\left[\frac{q^{\mu}+q^{-\mu}}{2}-\frac{W(1)+W(-1)}{2 K}\right]^{2} } \\
& +\frac{1}{\left(q^{\mu}-q^{-\mu}\right)^{2}}\left[e_{1}+\frac{q^{\mu}+q^{-\mu}}{2} \frac{W(1)-W(-1)}{K}\right]^{2},
\end{aligned}
$$

we can factorize the resulting four terms and arrive at (5.5). To find (5.6), we start with (4.44) and use our previous results for (5.4) and (5.3). We note that the terms linear in $x$ cancel identically, as they must, and after some factorization we deduce (5.6).

It is the coefficients (5.2)-(5.3) along with first-order divided-difference equation (4.31) that constitutes the structural relation for the Askey-Wilson polynomials, that is, the analogue of first-order difference or differential relations for the classical orthogonal polynomials. The divided-difference relations reported in [58] are all of second order.

It is an easy task to evaluate the Askey-Wilson integral (see [4], [56]) as a $q$-factorial and therefore compute the moments. The Askey-Wilson integral is defined by

$$
I_{2}\left(a_{1}, a_{2}, a_{3}, a_{4}\right):=\int_{\mathbb{T}} \frac{d z}{2 \pi i z} \frac{\left(z^{ \pm 2} ; q\right)_{\infty}}{\prod_{j=1}^{4}\left(a_{j} z^{ \pm 1} ; q\right)_{\infty}},
$$

with $\left|a_{j}\right|<1$ for $j=1, \ldots, 4$. 
Our method is to apply the general system of moment recurrences to the case $M=2$, and we find that it coincides with the recurrence of Kalnins and Miller [53] and Koelink and Koornwinder [57].

Proposition 5.2 ([97, Section 3.2]). The Askey-Wilson integral satisfies the two-term linear recurrence

$$
\begin{aligned}
& \left(\sigma_{4}-1\right) I_{2}\left(q a_{1}, a_{2}, a_{3}, a_{4}\right) \\
& \quad=\left(a_{1} a_{2}-1\right)\left(a_{1} a_{3}-1\right)\left(a_{1} a_{4}-1\right) I_{2}\left(a_{1}, a_{2}, a_{3}, a_{4}\right),
\end{aligned}
$$

which is solved by

$$
I_{2}\left(a_{1}, a_{2}, a_{3}, a_{4}\right)=2 \frac{\left(\sigma_{4} ; q\right)_{\infty}}{(q ; q)_{\infty} \prod_{k>j}\left(a_{j} a_{k} ; q\right)_{\infty}}
$$

Consequently, the moments are given by

$$
\begin{aligned}
m_{0, n}\left(a_{1}\right) & =\pi \frac{\left(a_{1} a_{2}, a_{1} a_{3}, a_{1} a_{4} ; q\right)_{n}}{\left(\sigma_{4} ; q\right)_{n}} I_{2}\left(a_{1}, a_{2}, a_{3}, a_{4}\right) \\
& =2 \pi \frac{\left(q^{n} \sigma_{4} ; q\right)_{\infty}}{\left(q^{n} a_{1} a_{2}, q^{n} a_{1} a_{3}, q^{n} a_{1} a_{4}, a_{2} a_{3}, a_{2} a_{4}, a_{3} a_{4}, q ; q\right)_{\infty}}
\end{aligned}
$$

Proof. We only require the $k=0$ case of (4.11) with $a=a_{1}$, and from (5.7) we compute the relevant coefficients as $\delta_{2,1}(a)=q^{-1} a^{-1}\left(1-\sigma_{4}\right)$ and $\delta_{2,0}(a)=q^{-1}\left(a+a^{-1}\right)\left(\sigma_{4}-1\right)+q^{-1}\left(\sigma_{1}-\sigma_{3}\right)$. The solution of the recurrence (5.24) follows from the arguments given in [57].

The specialization of the second-order divided-difference equation (4.63) with the $M=2$ spectral coefficients of Proposition 5.1 is given by

$$
\begin{aligned}
& \frac{\prod_{j=1}^{4}\left(1-a_{j} z\right)}{q z^{2}-1}\left[\left(E_{x}^{+}\right)^{2} p_{n}-p_{n}\right] \\
& \quad+\frac{\prod_{j=1}^{4}\left(z-a_{j}\right)}{z^{2}-q}\left[\left(E_{x}^{-}\right)^{2} p_{n}-p_{n}\right] \\
& \quad+q^{-n-1}\left(1-q^{n}\right)\left(q^{n} \sigma_{4}-q\right)\left(z^{2}-1\right) p_{n}=0 .
\end{aligned}
$$

This is soluble in terms of basic hypergeometric functions, and their polynomial solutions have been given by [60] or using factorization methods in 
[7], [11], and [10] as Askey-Wilson polynomials. The Askey-Wilson polynomials have an explicit form as a balanced ${ }_{4} \varphi_{3}$ function (see [56]) with the manifest symmetry under $z \leftrightarrow z^{-1}$ and are given in the monic form by

$$
\pi_{n}(x)=\frac{\left(a_{1} a_{2}, a_{1} a_{3}, a_{1} a_{4} ; q\right)_{n}}{\left(2 a_{1}\right)^{n}\left(q^{n-1} \sigma_{4} ; q\right)_{n}}{ }_{4} \varphi_{3}\left(\begin{array}{c}
q^{-n}, \sigma_{4} q^{n-1}, a_{1} z, a_{1} z^{-1} \\
a_{1} a_{2}, a_{1} a_{3}, a_{1} a_{4}
\end{array} ; q, q\right)
$$

or the alternative form (see [34]) which is manifestly symmetric under permutations of $a_{1}, a_{2}, a_{3}, a_{4}$

$$
\begin{aligned}
\pi_{n}(x)= & (2 z)^{-n} \frac{\left(a_{1} z, a_{2} z, a_{3} z, a_{4} z, \sigma_{4} q^{-1} ; q\right)_{n}}{\left(z^{2} ; q\right)_{n}\left(\sigma_{4} q^{-1} ; q\right)_{2 n}} \\
& \times{ }_{8} W_{7}\left(q^{-n} z^{-2} ; q^{-n}, a_{1} z^{-1}, a_{2} z^{-1}, a_{3} z^{-1}, a_{4} z^{-1} ; \frac{q^{2-n}}{\sigma_{4}}\right) .
\end{aligned}
$$

From $[82,(2.6)]$, or equivalently from [45, (4.18)] with $\alpha=0$, and [31, (III.23)] we deduce the expression for the Stieltjes function as a very-wellpoised ${ }_{8} W_{7}$

$$
\begin{aligned}
f(x)= & \frac{4 \pi\left(q^{-1} \sigma_{4} ; q\right)_{\infty}}{(q ; q)_{\infty} \prod_{k>j}\left(a_{j} a_{k} ; q\right)_{\infty}} \frac{\left(1-q z^{-2}\right)}{z \prod_{1 \leq j \leq 4}\left(1-a_{j} z^{-1}\right)} \\
& \times{ }_{8} W_{7}\left(q z^{-2} ; \frac{q}{a_{1}} z^{-1}, \frac{q}{a_{2}} z^{-1}, \frac{q}{a_{3}} z^{-1}, \frac{q}{a_{4}} z^{-1}, q ; q, q^{-1} \sigma_{4}\right),
\end{aligned}
$$

which exhibits the parameter symmetry. This expression is the one valid on the second Riemann sheet of the cut $x$-plane or exterior to the unit circle in $z,|z|>1$. There are evaluations of $q_{n}(x)$ in [82] and [45] that are simple generalizations of the above formulas, but we do not need to discuss them here.

Having an explicit form for the Stieltjes function means that we have two tasks at hand - to verify that is satisfies both the inhomogeneous divideddifference equation (4.13) and the large $x$ expansion formula (3.46).

Proposition 5.3. The Stieltjes function given by equation (5.31) satisfies the inhomogeneous divided-difference equation (4.13) with the constant

$$
U=\frac{8 \pi}{q-1} \frac{\left(q^{-1} \sigma_{4} ; q\right)_{\infty}}{(q ; q)_{\infty} \prod_{k>j}\left(a_{j} a_{k} ; q\right)_{\infty}} .
$$


Proof. We require an alternative form

$$
\begin{aligned}
f(x)= & 4 \pi \frac{q a_{1}}{\sigma_{4}} \frac{1}{\left(\frac{q}{a_{2} a_{3}}, \frac{q}{a_{2} a_{4}}, \frac{q}{a_{3} a_{4}} ; q\right)_{\infty}} \\
& \times\left\{-\frac{\left(\frac{q}{a_{2} z}, \frac{q}{a_{3} z}, \frac{q}{a_{4} z}, \frac{\sigma_{4}}{q a_{1} z}, \frac{q^{2} a_{1} z}{\sigma_{4}} ; q\right)_{\infty}}{\left(a_{2} a_{3}, a_{2} a_{4}, a_{3} a_{4}, a_{1} z^{-1}, a_{2} z^{-1}, a_{3} z^{-1}, a_{4} z^{-1}, a_{1} z ; q\right)_{\infty}}\right. \\
& +\frac{\left(q^{-1} \sigma_{4}, q \frac{a_{1}}{a_{2}}, q \frac{a_{1}}{a_{3}}, q \frac{a_{1}}{a_{4}}, \frac{q^{2}}{\sigma_{4}} ; q\right)_{\infty}}{\left(q, q a_{1}^{2} ; q\right)_{\infty} \prod_{k>j}\left(a_{j} a_{k} ; q\right)_{\infty}\left(1-a_{1} z\right)\left(1-a_{1} z^{-1}\right)} \\
& \left.\times{ }_{8} W_{7}\left(a_{1}^{2} ; a_{1} z, a_{1} z^{-1}, a_{1} a_{2}, a_{1} a_{3}, a_{1} a_{4} ; \frac{q^{2}}{\sigma_{4}}\right)\right\}
\end{aligned}
$$

which makes the $z \leftrightarrow z^{-1}$ symmetry manifest in the second term only. This is derived from the previous expression by utilizing [31, (III.37)] combined with [31, (III.23)], and [31, (III.36)] for the term which specializes. We are now in a position to verify that (5.33) satisfies (4.13). This relies on the fact that (4.13) with the data (5.7) is precisely the contiguous relation, [45, $(2.2)]$, which in our context states

$$
\begin{aligned}
z^{2} \frac{\prod_{j=2,3,4}\left(1-q^{-1 / 2} a_{j} z^{-1}\right)}{1-q^{1 / 2} a_{1} z} \\
\quad \times{ }_{8} W_{7}\left(a_{1}^{2} ; q^{1 / 2} a_{1} z, q^{-1 / 2} a_{1} z^{-1}, a_{1} a_{2}, a_{1} a_{3}, a_{1} a_{4} ; \frac{q^{2}}{\sigma_{4}}\right) \\
\quad-z^{-2} \frac{\prod_{j=2,3,4}\left(1-q^{-1 / 2} a_{j} z\right)}{1-q^{1 / 2} a_{1} z^{-1}} \\
\quad \times{ }_{8} W_{7}\left(a_{1}^{2} ; q^{-1 / 2} a_{1} z, q^{1 / 2} a_{1} z^{-1}, a_{1} a_{2}, a_{1} a_{3}, a_{1} a_{4} ; \frac{q^{2}}{\sigma_{4}}\right) \\
=q^{-3 / 2} a_{2} a_{3} a_{4}\left(1-\frac{q}{\sigma_{4}}\right)\left(z-z^{-1}\right) \\
\quad \times{ }_{8} W_{7}\left(a_{1}^{2} ; q^{1 / 2} a_{1} z, q^{1 / 2} a_{1} z^{-1}, a_{1} a_{2}, a_{1} a_{3}, a_{1} a_{4} ; \frac{q}{\sigma_{4}}\right) .
\end{aligned}
$$

We have also used the specialization formula when $a q=b c$

$$
{ }_{8} W_{7}\left(a ; b, c, d, e, f ; q, \frac{q^{2} a^{2}}{b c d e f}\right)=\frac{(a q, a q / d e, a q / d f, a q / e f ; q)_{\infty}}{(a q / d, a q / e, a q / f, a q / \operatorname{def} ; q)_{\infty}}
$$


which applies to the right-hand side of the previous equation. A consequence one can draw from this calculation is the explicit evaluation of the constant spectral coefficient.

REMARK 5.1. The constant $U$ can be found in another way. This is by noting that the initial condition for $\Phi_{0}$ is given by (4.39) and that $\Phi_{n}$ is given purely by the leading order term in (5.4), so that knowing the normalization $\gamma_{0}^{2}$ allows us to make the evaluation.

Proposition 5.4. The Stieltjes function possesses the explicit large $x$ moment-generating function formula

$$
\begin{aligned}
f(x)= & -\frac{4 \pi a_{1}\left(\sigma_{4} ; q\right)_{\infty}}{(q ; q)_{\infty} \prod_{k>j}\left(a_{j} a_{k} ; q\right)_{\infty}\left(1-a_{1} z\right)\left(1-a_{1} z^{-1}\right)} \\
& \times{ }_{4} \varphi_{3}\left(\begin{array}{c}
q, a_{1} a_{2}, a_{1} a_{3}, a_{1} a_{4} \\
q a_{1} z, q a_{1} z^{-1}, \sigma_{4}
\end{array} ;, q\right) \\
& -\frac{4 \pi\left(a_{2} a_{3} a_{4} z^{-1}, q z^{-2} ; q\right)_{\infty}}{z\left(a_{2} a_{3}, a_{2} a_{4}, a_{3} a_{4}, a_{2} z^{-1}, a_{3} z^{-1}, a_{4} z^{-1} ; q\right)_{\infty}} \frac{1}{\phi_{\infty}\left(x ; a_{1}\right)} \\
& \times{ }_{3} \varphi_{2}\left(\begin{array}{c}
a_{2} z^{-1}, a_{3} z^{-1}, a_{4} z^{-1} \\
\left.a_{2} a_{3} a_{4} z^{-1}, q z^{-2} ; q, q\right) .
\end{array}\right.
\end{aligned}
$$

Proof. By applying the transformation formula of [31, (III.36)] to the ${ }_{8} W_{7}$ function in (5.33), we get a sum of two ${ }_{4} \varphi_{3}$ functions one of which reduces to a ${ }_{3} \varphi_{2}$ function. After some simplification, we get (5.36). We observe that the ${ }_{4} \varphi_{3}$ term is precisely the second term of (3.46) given our formula for the moments (5.26). This allows us to conclude that

$$
\begin{aligned}
f_{\infty}(x)= & -\frac{4 \pi\left(a_{2} a_{3} a_{4} z^{-1}, q z^{-2} ; q\right)_{\infty}}{z\left(a_{2} a_{3}, a_{2} a_{4}, a_{3} a_{4}, a_{2} z^{-1}, a_{3} z^{-1}, a_{4} z^{-1} ; q\right)_{\infty}} \\
& \times{ }_{3} \varphi_{2}\left(\begin{array}{c}
a_{2} z^{-1}, a_{3} z^{-1}, a_{4} z^{-1} \\
\left.a_{2} a_{3} a_{4} z^{-1}, q z^{-2} ; q, q\right) .
\end{array}\right.
\end{aligned}
$$

\section{§6. Deformation differences}

In this section we discuss the deformation structures based upon a nonlinear lattice in the $u$ variable (or any number of variables for that matter) and the corresponding divided-difference operators. We emphasize that many of the results for the spectral structure have a parallel result in the deformation structure, although there will be crucial differences in the details. Denote the 
deformation variable $u$ and its forward and backward shifts by $v_{ \pm}=E_{u}^{ \pm} u$ and their difference by $\Delta v:=v_{+}-v_{-}$. The reader should be aware that the deformation lattice does not have to be the same type as the spectral lattice. Our $\mathbb{D}$-semiclassical weight $w$, defined by Definition 4.1 , acquires an additional dependence on a deformation variable $u$, and furthermore satisfies a deformation divided-difference equation with respect to $u$.

Definition 6.1. The deformed $\mathbb{D}$-semiclassical weight satisfies

$$
R \mathbb{D}_{u} w=2 S \mathbb{M}_{u} w
$$

or equivalently

$$
\frac{w\left(x ; v_{+}\right)}{w\left(x ; v_{-}\right)}=\frac{R+\Delta v S}{R-\Delta v S}(x ; u),
$$

for polynomials $S(x ; u), R(x ; u)$ irreducible in $x$ and $u \in J$. In addition, we assume that $R \pm \Delta v S \neq 0$ for all $x \in G, u \in J$ and that the deformed OPS exists, that is, that $\gamma_{n}\left(v_{ \pm}\right) \neq 0$ for all $n \in \mathbb{Z}_{\geq 0}$. We also require the condition that $\gamma_{0}\left(v_{+}\right)(R-\Delta v S)+\gamma_{0}\left(v_{-}\right)(R+\Delta v S) \neq 0$.

There is a very simple motivation for this relation. In the process of extending the weights beyond those of the classical cases in the Askey table by increasing the degrees $\operatorname{deg}_{x} W \geq 3, \operatorname{deg}_{x} V \geq 2$, the weights acquire additional parameters which if suitably chosen sit in a completely reflexive or symmetric way to the spectral variable, and therefore will satisfy an analogous linear, homogeneous, and first-order divided-difference equation in any of these new parameters.

There are conditions imposed on the weight given that it satisfies both (4.1) or (4.2) and (6.1) or (6.2).

Proposition 6.1. The spectral data polynomials $W(x ; u), V(x ; u)$ and the deformation data polynomials $R(x ; u), S(x ; u)$ satisfy the compatibility relations

$$
\begin{aligned}
& {\left[\mathbb{D}_{x} \frac{2 S}{R}-\mathbb{D}_{u} \frac{2 V}{W}\right]\left[1-\frac{1}{16} \Delta y^{2} \Delta v^{2} \mathbb{D}_{x} \frac{2 S}{R} \mathbb{D}_{u} \frac{2 V}{W}\right]} \\
& \quad=\frac{1}{4} \Delta y^{2} \mathbb{D}_{x} \frac{2 S}{R}\left(\mathbb{M}_{u} \frac{2 V}{W}\right)^{2}-\frac{1}{4} \Delta v^{2} \mathbb{D}_{u} \frac{2 V}{W}\left(\mathbb{M}_{x} \frac{2 S}{R}\right)^{2},
\end{aligned}
$$


or alternatively

$$
\begin{aligned}
& \frac{W+\Delta y V}{W-\Delta y V}\left(x ; v_{+}\right) \frac{R+\Delta v S}{R-\Delta v S}\left(y_{-} ; u\right) \\
& \quad=\frac{W+\Delta y V}{W-\Delta y V}\left(x ; v_{-}\right) \frac{R+\Delta v S}{R-\Delta v S}\left(y_{+} ; u\right)
\end{aligned}
$$

Proof. From the fact that the weight satisfies the overdetermined system of first-order divided-difference equations (4.1) and (6.1), we first compute that

$$
\begin{aligned}
& {\left[1-\frac{1}{16} \Delta y^{2} \Delta v^{2} \mathbb{D}_{x} \frac{2 S}{R} \mathbb{D}_{u} \frac{2 V}{W}\right] \mathbb{M}_{u} \mathbb{D}_{x} w} \\
& =\left[\mathbb{M}_{u} \frac{2 V}{W}+\frac{1}{4} \Delta v^{2} \mathbb{D}_{u} \frac{2 V}{W} \mathbb{M}_{x} \frac{2 S}{R}\right] \mathbb{M}_{u} \mathbb{M}_{x} w \\
& {\left[1-\frac{1}{16} \Delta y^{2} \Delta v^{2} \mathbb{D}_{x} \frac{2 S}{R} \mathbb{D}_{u} \frac{2 V}{W}\right] \mathbb{M}_{x} \mathbb{D}_{u} w} \\
& \quad=\left[\mathbb{M}_{x} \frac{2 S}{R}+\frac{1}{4} \Delta y^{2} \mathbb{D}_{x} \frac{2 S}{R} \mathbb{M}_{u} \frac{2 V}{W}\right] \mathbb{M}_{x} \mathbb{M}_{u} w
\end{aligned}
$$

using (2.11). Now we compute

$$
\begin{aligned}
& \mathbb{D}_{u} \mathbb{D}_{x} w=\mathbb{D}_{u} \frac{2 V}{W} \mathbb{M}_{u} \mathbb{M}_{x} w+\mathbb{M}_{u} \frac{2 V}{W} \mathbb{D}_{u} \mathbb{M}_{x} w, \\
& \mathbb{D}_{x} \mathbb{D}_{u} w=\mathbb{D}_{x} \frac{2 S}{R} \mathbb{M}_{x} \mathbb{M}_{u} w+\mathbb{M}_{x} \frac{2 S}{R} \mathbb{D}_{x} \mathbb{M}_{u} w
\end{aligned}
$$

using (2.10), and we use the results of the previous set of equations to derive two independent relations linking $\mathbb{D}_{u} \mathbb{D}_{x} w$ and $\mathbb{M}_{u} \mathbb{M}_{x} w$. A comparison of these two latter relations leads to (6.3).

To establish (6.4), we note that

$$
\begin{aligned}
w\left(y_{+} ; v_{+}\right) & =\frac{W+\Delta y V}{W-\Delta y V}\left(x ; v_{+}\right) w\left(y_{-} ; v_{+}\right) \\
& =\frac{W+\Delta y V}{W-\Delta y V}\left(x ; v_{+}\right) \frac{R+\Delta v S}{R-\Delta v S}\left(y_{-} ; u\right) w\left(y_{-} ; v_{-}\right),
\end{aligned}
$$

whereas

$$
\begin{aligned}
w\left(y_{+} ; v_{+}\right) & =\frac{R+\Delta v S}{R-\Delta v S}\left(y_{+} ; u\right) w\left(y_{+} ; v_{-}\right) \\
& =\frac{R+\Delta v S}{R-\Delta v S}\left(y_{+} ; u\right) \frac{W+\Delta y V}{W-\Delta y V}\left(x ; v_{-}\right) w\left(y_{-} ; v_{-}\right) .
\end{aligned}
$$


Proposition 6.2. As a consequence of Definition 6.1, the Stieltjes transform satisfies the inhomogeneous equation

$$
R \mathbb{D}_{u} f=2 S \mathbb{M}_{u} f+T .
$$

The deformed $\mathbb{D}$-semiclassical class of orthogonal polynomial systems are characterized by the property that $R(x ; u), S(x ; u)$ and $T(x ; u)$ in (6.11) are polynomials in $x$.

Proof. From the definition (3.19) and (6.1), we compute

$$
\mathbb{D}_{u} f(x ; u)=\int \mathbb{D} y \frac{\mathbb{M}_{u} w(y ; u)}{x-y} \frac{2 S(y ; u)}{R(y ; u)} .
$$

Now we observe that the rational function $2 S(y ; u) /[(x-y) R(y ; u)]$ has the partial fraction expansion

$$
\frac{2 S(y ; u)}{(x-y) R(y ; u)}=\frac{2 S(x ; u)}{(x-y) R(x ; u)}+\sum_{j, R\left(x_{j}\right)=0} \frac{2 S\left(x_{j} ; u\right)}{R^{\prime}\left(x_{j} ; u\right)\left(x-x_{j}\right)} \frac{1}{y-x_{j}}
$$

Consequently,

$$
\mathbb{D}_{u} f(x ; u)=\frac{2 S(x ; u)}{R(x ; u)} \int \mathbb{D} y \frac{\mathbb{M}_{u} w(y ; u)}{x-y}
$$

$$
\begin{aligned}
& +\sum_{j, R\left(x_{j}\right)=0} \frac{2 S\left(x_{j} ; u\right)}{R^{\prime}\left(x_{j} ; u\right)\left(x-x_{j}\right)} \int \mathbb{D} y \frac{\mathbb{M}_{u} w(y ; u)}{y-x_{j}} \\
= & \frac{2 S(x ; u)}{R(x ; u)} \mathbb{M}_{u} f(x ; u)-\sum_{j, R\left(x_{j}\right)=0} \frac{2 S\left(x_{j} ; u\right)}{R^{\prime}\left(x_{j} ; u\right)\left(x-x_{j}\right)} \mathbb{M}_{u} f\left(x_{j} ; u\right) .
\end{aligned}
$$

We conclude that (6.11) follows with $\operatorname{deg}_{x} T \leq \operatorname{deg}_{x} R-1$.

Proposition 6.3. The compatibility of (6.11) and (4.13) implies the following identity on $U$ and $T$ :

$$
\begin{aligned}
\Delta y[ & \frac{(W+\Delta y V)\left(x ; v_{+}\right)}{(W+\Delta y V)\left(x ; v_{-}\right)}(R+\Delta v S)\left(y_{-} ; u\right) U\left(x ; v_{-}\right) \\
& \left.-(R-\Delta v S)\left(y_{-} ; u\right) U\left(x ; v_{+}\right)\right] \\
= & \Delta v\left[(W+\Delta y V)\left(x ; v_{+}\right) T\left(y_{-} ; u\right)\right. \\
& \left.-(W-\Delta y V)\left(x ; v_{+}\right) \frac{(R-\Delta v S)\left(y_{-} ; u\right)}{(R-\Delta v S)\left(y_{+} ; u\right)} T\left(y_{+} ; u\right)\right] .
\end{aligned}
$$


Proof. We begin by defining the ratio

$$
\begin{aligned}
\chi & \equiv \frac{(W+\Delta y V)\left(x ; v_{+}\right)}{(W+\Delta y V)\left(x ; v_{-}\right)} \frac{(R+\Delta v S)\left(y_{-} ; u\right)}{(R+\Delta v S)\left(y_{+} ; u\right)} \\
& =\frac{(W-\Delta y V)\left(x ; v_{+}\right)}{(W-\Delta y V)\left(x ; v_{-}\right)} \frac{(R-\Delta v S)\left(y_{-} ; u\right)}{(R-\Delta v S)\left(y_{+} ; u\right)}
\end{aligned}
$$

by virtue of (6.4). Consider (4.13) in the form

$$
\begin{aligned}
& (W-\Delta y V)(x ; u) f\left(y_{+} ; u\right) \\
& \quad-(W+\Delta y V)(x ; u) f\left(y_{-} ; u\right)-\Delta y U(x ; u)=0,
\end{aligned}
$$

and construct the combination $(W+\Delta y V)\left(x ; v_{+}\right) /(W+\Delta y V)\left(x ; v_{-}\right)(R+$ $\Delta v S)\left(y_{-} ; u\right) \times(6.18)\left(u \mapsto v_{-}\right)-(R-\Delta v S)\left(y_{-} ; u\right) \times(6.18)\left(u \mapsto v_{+}\right)$. Using the above identity, we find that the two terms containing $f$ possess the factors $(R+\Delta v S)\left(y_{-} ; u\right) f\left(y_{-} ; v_{-}\right)-(R-\Delta v S)\left(y_{-} ; u\right) f\left(y_{-} ; v_{+}\right)$and $(R+$ $\Delta v S)\left(y_{+} ; u\right) f\left(y_{+} ; v_{-}\right)-(R-\Delta v S)\left(y_{+} ; u\right) f\left(y_{+} ; v_{+}\right)$, into which we can apply (6.11) in the form

$$
(R-\Delta v S)(x ; u) f\left(x ; v_{+}\right)-(R+\Delta v S)(x ; u) f\left(x ; v_{-}\right)-\Delta v T(x ; u)=0 .
$$

Then (6.16) immediately follows.

We can extend the notion of a generic or regular $\mathbb{D}$-semiclassical weight, given in Definition 4.2, to the deformed situation by the following definition.

Definition 6.2. A regular, deformed $\mathbb{D}$-semiclassical weight is one that satisfies the requirements of Definition 4.2 and $\operatorname{deg}_{x} R=\operatorname{deg}_{x} S=L$ and $\operatorname{deg}_{x} T=\operatorname{deg}_{x} R-1$. Clearly $M$ in Definition 4.2 and $L$ are related, depending on the specific case on hand.

The analogue of Proposition 4.2 is the following.

Proposition 6.4. Let the deformation coefficients $R_{n}(x ; u), \Gamma_{n}(x ; u)$, $\Xi_{n}(x ; u), \Phi_{n}(x ; u), \Psi_{n}(x ; u)$ be defined in terms of bilinear formulas involving products of the polynomials and associated functions by

$$
\frac{2}{H_{n}} R_{n}=(R+\Delta v S)\left[-\frac{1}{a_{n}\left(v_{-}\right)}+p_{n-1}\left(; v_{+}\right) q_{n}\left(; v_{-}\right)-p_{n}\left(; v_{+}\right) q_{n-1}\left(; v_{-}\right)\right]
$$

$$
+(R-\Delta v S)\left[-\frac{1}{a_{n}\left(v_{+}\right)}+p_{n-1}\left(; v_{-}\right) q_{n}\left(; v_{+}\right)\right.
$$




$$
\begin{aligned}
& \left.-p_{n}\left(; v_{-}\right) q_{n-1}\left(; v_{+}\right)\right] \\
\frac{\Delta v}{H_{n}} \Gamma_{n}= & (R+\Delta v S)\left[\frac{1}{a_{n}\left(v_{-}\right)}-p_{n-1}\left(; v_{+}\right) q_{n}\left(; v_{-}\right)-p_{n}\left(; v_{+}\right) q_{n-1}\left(; v_{-}\right)\right] \\
(6.21) \quad & (R-\Delta v S)\left[-\frac{1}{a_{n}\left(v_{+}\right)}+p_{n-1}\left(; v_{-}\right) q_{n}\left(; v_{+}\right)\right. \\
& \left.+p_{n}\left(; v_{-}\right) q_{n-1}\left(; v_{+}\right)\right], \\
\frac{\Delta v}{H_{n}} \Xi_{n}= & (R+\Delta v S)\left[\frac{1}{a_{n}\left(v_{-}\right)}+p_{n-1}\left(; v_{+}\right) q_{n}\left(; v_{-}\right)+p_{n}\left(; v_{+}\right) q_{n-1}\left(; v_{-}\right)\right] \\
(6.22) & +(R-\Delta v S)\left[-\frac{1}{a_{n}\left(v_{+}\right)}-p_{n-1}\left(; v_{-}\right) q_{n}\left(; v_{+}\right)\right. \\
& \left.-p_{n}\left(; v_{-}\right) q_{n-1}\left(; v_{+}\right)\right],
\end{aligned}
$$$$
\frac{\Delta v}{2 H_{n}} \Phi_{n}=(R+\Delta v S) p_{n}\left(; v_{+}\right) q_{n}\left(; v_{-}\right)
$$

$$
-(R-\Delta v S) p_{n}\left(; v_{-}\right) q_{n}\left(; v_{+}\right)
$$

and

$$
\begin{aligned}
\frac{\Delta v}{2 H_{n}} \Psi_{n}= & -(R+\Delta v S) p_{n-1}\left(; v_{+}\right) q_{n-1}\left(; v_{-}\right) \\
& +(R-\Delta v S) p_{n-1}\left(; v_{-}\right) q_{n-1}\left(; v_{+}\right)
\end{aligned}
$$

for $n \geq 0$ and where the decoupling factor $H_{n}(u)$ has $\operatorname{deg}_{x} H_{n}=0$. Then the deformation coefficients of the deformed $\mathbb{D}$-semiclassical class are polynomials in $x$.

Proof. We offer a proof in the spirit of that for Proposition 4.2, using Laguerre's method, and apply it to (6.23) first. From (6.11) and (3.21), we have

$$
\begin{aligned}
0 & =(R-\Delta v S) f\left(; v_{+}\right)-(R+\Delta v S) f\left(; v_{-}\right)-\Delta v T \\
& =(R-\Delta v S) \frac{q_{n}+p_{n-1}^{(1)}}{p_{n}}\left(; v_{+}\right)-(R+\Delta v S) \frac{q_{n}+p_{n-1}^{(1)}}{p_{n}}\left(; v_{-}\right)-\Delta v T \\
(6.25) & =\frac{(R-\Delta v S) p_{n}\left(; v_{-}\right) p_{n-1}^{(1)}\left(; v_{+}\right)-(R+\Delta v S) p_{n}\left(; v_{+}\right) p_{n-1}^{(1)}\left(; v_{-}\right)}{p_{n}\left(; v_{+}\right) p_{n}\left(; v_{-}\right)}
\end{aligned}
$$




$$
\begin{aligned}
& -\frac{\Delta v T p_{n}\left(; v_{+}\right) p_{n}\left(; v_{-}\right)}{p_{n}\left(; v_{+}\right) p_{n}\left(; v_{-}\right)} \\
& +\frac{(R-\Delta v S) p_{n}\left(; v_{-}\right) q_{n}\left(; v_{+}\right)-(R+\Delta v S) p_{n}\left(; v_{+}\right) q_{n}\left(; v_{-}\right)}{p_{n}\left(; v_{+}\right) p_{n}\left(; v_{-}\right)}
\end{aligned}
$$

In this last expression, the numerator of the first two terms is clearly a polynomial in $x$ which implies that the numerator of the third term is likewise. This latter numerator is an odd function under the exchange $v_{+} \leftrightarrow v_{-}$and we denote it by $\Delta v \Phi_{n}(x ; u) /\left(2 H_{n}\right)$, thus deriving (6.23). The relation (6.24) is simply the case of (6.23) under $n \mapsto n-1$.

The remaining relations may now be derived from the following argument. From the workings of the previous paragraph, we know that

$$
\begin{aligned}
\frac{\Delta v \Phi_{n}}{2 H_{n}}= & (R-\Delta v S) p_{n}\left(; v_{-}\right) p_{n-1}^{(1)}\left(; v_{+}\right) \\
& -(R+\Delta v S) p_{n}\left(; v_{+}\right) p_{n-1}^{(1)}\left(; v_{-}\right)-\Delta v T p_{n}\left(; v_{+}\right) p_{n}\left(; v_{-}\right) .
\end{aligned}
$$

By multiplying the left-hand side of this relation by the Casoratian $a_{n}\left(v_{+}\right) \times$ $\left[p_{n-1} p_{n-1}^{(1)}-p_{n} p_{n-2}^{(1)}\right]\left(; v_{+}\right)=1$, we observe that the resulting equation separates into two terms with the factorization

$$
\begin{aligned}
& p_{n}\left(; v_{+}\right)\left[-a_{n}\left(v_{+}\right) \frac{\Delta v \Phi_{n}}{2 H_{n}} p_{n-2}^{(1)}\left(; v_{+}\right)\right. \\
& \left.\quad+(R+\Delta v S) p_{n-1}^{(1)}\left(; v_{-}\right)+\Delta v T p_{n}\left(; v_{-}\right)\right] \\
& \quad=p_{n-1}^{(1)}\left(; v_{+}\right)\left[-a_{n}\left(v_{+}\right) \frac{\Delta v \Phi_{n}}{2 H_{n}} p_{n-1}\left(; v_{+}\right)+(R-\Delta v S) p_{n}\left(; v_{-}\right)\right]
\end{aligned}
$$

which implies that this expression contains the polynomial factors $p_{n}\left(; v_{+}\right) \times$ $p_{n-1}^{(1)}\left(; v_{+}\right)$and therefore can be written as $p_{n}\left(; v_{+}\right) p_{n-1}^{(1)}\left(; v_{+}\right) \pi_{1}$, where $\pi_{1}$ is a polynomial in $x$. Given that the two factors $p_{n}\left(; v_{+}\right), p_{n-1}^{(1)}\left(; v_{+}\right)$are nonzero, this then leads to two evaluations for $\pi_{1}$, which by constructing a suitable combination of these and employing the Casoratian once more we find, upon some simplification,

$$
\begin{aligned}
\pi_{1}= & a_{n}\left(v_{+}\right)\left[-(R+\Delta v S) p_{n-1}\left(; v_{+}\right) q_{n}\left(; v_{-}\right)\right. \\
& \left.+(R-\Delta v S) p_{n}\left(; v_{-}\right) q_{n-1}\left(; v_{+}\right)\right] .
\end{aligned}
$$


Alternatively, we could have multiplied the left-hand side of (6.26) by the Casoratian $a_{n}\left(v_{-}\right)\left[p_{n-1} p_{n-1}^{(1)}-p_{n} p_{n-2}^{(1)}\right]\left(; v_{-}\right)=1$ and deduced the factorization

$$
\begin{aligned}
p_{n}\left(; v_{-}\right) & {\left[-a_{n}\left(v_{-}\right) \frac{\Delta v \Phi_{n}}{2 H_{n}} p_{n-2}^{(1)}\left(; v_{-}\right)\right.} \\
& \left.-(R-\Delta v S) p_{n-1}^{(1)}\left(; v_{+}\right)+\Delta v T p_{n}\left(; v_{+}\right)\right] \\
& =p_{n-1}^{(1)}\left(; v_{-}\right)\left[-a_{n}\left(v_{-}\right) \frac{\Delta v \Phi_{n}}{2 H_{n}} p_{n-1}\left(; v_{-}\right)-(R+\Delta v S) p_{n}\left(; v_{+}\right)\right] .
\end{aligned}
$$

This fact then allows us to conclude that either of these expressions can be written as $p_{n}\left(; v_{-}\right) p_{n-1}^{(1)}\left(; v_{-}\right) \pi_{2}$, where $\pi_{2}$ is another polynomial in $x$. Using an identical procedure to that employed above, we can infer that

$$
\begin{aligned}
\pi_{2}= & a_{n}\left(v_{-}\right)\left[-(R+\Delta v S) p_{n}\left(; v_{+}\right) q_{n-1}\left(; v_{-}\right)\right. \\
& \left.+(R-\Delta v S) p_{n-1}\left(; v_{-}\right) q_{n}\left(; v_{+}\right)\right] .
\end{aligned}
$$

Relations (6.20) and (6.21) then follow from the definitions

$$
\begin{aligned}
& 2 R_{n}-\Delta v \Gamma_{n}+2 H_{n} \frac{R+\Delta v S}{a_{n}\left(v_{-}\right)}=-\frac{2 H_{n}}{a_{n}\left(v_{+}\right)} \pi_{1}, \\
& 2 R_{n}+\Delta v \Gamma_{n}+2 H_{n} \frac{R-\Delta v S}{a_{n}\left(v_{+}\right)}=\frac{2 H_{n}}{a_{n}\left(v_{-}\right)} \pi_{2},
\end{aligned}
$$

whereas (6.22) follows from $\Delta v\left(\Gamma_{n}+\Xi_{n}\right) / 2 H_{n}=(R+\Delta v S) / a_{n}\left(v_{-}\right)-(R-$ $\Delta v S) / a_{n}\left(v_{+}\right)$.

Analogous to the large $x$ expansions of the spectral coefficients, we have the following expansions for the deformation coefficients.

Proposition 6.5. Let $L=\max \left(\operatorname{deg}_{x} R, \operatorname{deg}_{x} S\right)$. As $x \rightarrow \infty$, we have the leading orders of the terminating expansions of the deformation coefficients

$$
\begin{aligned}
\frac{2}{H_{n}} R_{n}= & -\left(\gamma_{n}\left(v_{+}\right)+\gamma_{n}\left(v_{-}\right)\right)\left[\frac{R-\Delta v S}{\gamma_{n-1}\left(v_{+}\right)}+\frac{R+\Delta v S}{\gamma_{n-1}\left(v_{-}\right)}\right] \\
& +\sum_{i=0}^{n-1}\left(b_{i}\left(v_{+}\right)-b_{i}\left(v_{-}\right)\right)\left[(R+\Delta v S) \frac{\gamma_{n}\left(v_{+}\right)}{\gamma_{n-1}\left(v_{-}\right)}\right. \\
& \left.-(R-\Delta v S) \frac{\gamma_{n}\left(v_{-}\right)}{\gamma_{n-1}\left(v_{+}\right)}\right] x^{-1}+\mathrm{O}\left(x^{L-2}\right), \quad n \geq 0
\end{aligned}
$$


$(6.33)$

$$
\begin{aligned}
\frac{\Delta v}{2 H_{n}} \Phi_{n}= & {\left[(R+\Delta v S) \frac{\gamma_{n}\left(v_{+}\right)}{\gamma_{n}\left(v_{-}\right)}-(R-\Delta v S) \frac{\gamma_{n}\left(v_{-}\right)}{\gamma_{n}\left(v_{+}\right)}\right] x^{-1} } \\
& +\left((R+\Delta v S) \frac{\gamma_{n}\left(v_{+}\right)}{\gamma_{n}\left(v_{-}\right)}\left[\sum_{i=0}^{n} b_{i}\left(v_{-}\right)-\sum_{i=0}^{n-1} b_{i}\left(v_{+}\right)\right]\right. \\
& \left.+(R-\Delta v S) \frac{\gamma_{n}\left(v_{-}\right)}{\gamma_{n}\left(v_{+}\right)}\left[\sum_{i=0}^{n-1} b_{i}\left(v_{-}\right)-\sum_{i=0}^{n} b_{i}\left(v_{+}\right)\right]\right) x^{-2} \\
& +\mathrm{O}\left(x^{L-3}\right), \quad n \geq 0
\end{aligned}
$$

$$
\begin{aligned}
\frac{\Delta v}{2 H_{n}} \Psi_{n}= & -\left[(R+\Delta v S) \frac{\gamma_{n-1}\left(v_{+}\right)}{\gamma_{n-1}\left(v_{-}\right)}-(R-\Delta v S) \frac{\gamma_{n-1}\left(v_{-}\right)}{\gamma_{n-1}\left(v_{+}\right)}\right] x^{-1} \\
& -\left((R+\Delta v S) \frac{\gamma_{n-1}\left(v_{+}\right)}{\gamma_{n-1}\left(v_{-}\right)}\left[\sum_{i=0}^{n-1} b_{i}\left(v_{-}\right)-\sum_{i=0}^{n-2} b_{i}\left(v_{+}\right)\right]\right.
\end{aligned}
$$

$$
\begin{aligned}
& \left.+(R-\Delta v S) \frac{\gamma_{n-1}\left(v_{-}\right)}{\gamma_{n-1}\left(v_{+}\right)}\left[\sum_{i=0}^{n-2} b_{i}\left(v_{-}\right)-\sum_{i=0}^{n-1} b_{i}\left(v_{+}\right)\right]\right) x^{-2} \\
& +\mathrm{O}\left(x^{L-3}\right), \quad n \geq 0
\end{aligned}
$$

$$
\frac{\Delta v}{H_{n}} \Gamma_{n}=\left(\gamma_{n}\left(v_{-}\right)-\gamma_{n}\left(v_{+}\right)\right)\left[\frac{R+\Delta v S}{\gamma_{n-1}\left(v_{-}\right)}+\frac{R-\Delta v S}{\gamma_{n-1}\left(v_{+}\right)}\right]
$$

$$
\begin{aligned}
& +\sum_{i=0}^{n-1}\left(b_{i}\left(v_{+}\right)-b_{i}\left(v_{-}\right)\right)\left[(R+\Delta v S) \frac{\gamma_{n}\left(v_{+}\right)}{\gamma_{n-1}\left(v_{-}\right)}\right. \\
& \left.+(R-\Delta v S) \frac{\gamma_{n}\left(v_{-}\right)}{\gamma_{n-1}\left(v_{+}\right)}\right] x^{-1}+\mathrm{O}\left(x^{L-2}\right), \quad n \geq 0,
\end{aligned}
$$

and

$$
\begin{aligned}
\frac{\Delta v}{H_{n}} \Xi_{n}= & \left(\gamma_{n}\left(v_{-}\right)+\gamma_{n}\left(v_{+}\right)\right)\left[\frac{R+\Delta v S}{\gamma_{n-1}\left(v_{-}\right)}-\frac{R-\Delta v S}{\gamma_{n-1}\left(v_{+}\right)}\right] \\
& -\sum_{i=0}^{n-1}\left(b_{i}\left(v_{+}\right)-b_{i}\left(v_{-}\right)\right)\left[(R+\Delta v S) \frac{\gamma_{n}\left(v_{+}\right)}{\gamma_{n-1}\left(v_{-}\right)}\right. \\
& \left.+(R-\Delta v S) \frac{\gamma_{n}\left(v_{-}\right)}{\gamma_{n-1}\left(v_{+}\right)}\right] x^{-1}+\mathrm{O}\left(x^{L-2}\right), \quad n \geq 0 .
\end{aligned}
$$

Proof. These formulas follow from the substitution of the expansions (3.34) and (3.35) into the definitions (6.20)-(6.24). 
REMARK 6.1. Unlike the case of the spectral coefficients (see Proposition 4.3), the leading terms of the deformation coefficients are not determined by the weight data alone but depend nontrivially upon the three-term recurrence coefficients.

Corollary 6.1. In the regular, deformed $\mathbb{D}$-semiclassical case the deformation coefficients $R_{n}, \Gamma_{n}, \Phi_{n}, \Psi_{n}, \Xi_{n}$ are polynomials in the spectral variable $x$ with degrees independent of $n, \operatorname{deg}_{x} R_{n}=\operatorname{deg}_{x} \Gamma_{n}=\operatorname{deg}_{x} \Xi_{n}=$ $\max \left(\operatorname{deg}_{x} R, \operatorname{deg}_{x} S\right)$ and $\operatorname{deg}_{x} \Phi_{n}=\operatorname{deg}_{x} \Psi_{n}=\max \left(\operatorname{deg}_{x} R, \operatorname{deg}_{x} S\right)-1$.

The expressions for the deformation coefficients (6.24)-(6.24) can be inverted yielding a system of linear divided-difference equations in the deformation variable for the polynomials and associated functions.

Proposition 6.6. The OPS corresponding to a deformed $\mathbb{D}$-semiclassical weight satisfies the deformation divided-difference equation

$$
\mathbb{D}_{u} Y_{n}:=B_{n} \mathbb{M}_{u} Y_{n}=\frac{1}{R_{n}}\left(\begin{array}{ll}
\Gamma_{n} & \Phi_{n} \\
\Psi_{n} & \Xi_{n}
\end{array}\right) \mathbb{M}_{u} Y_{n}, \quad n \geq 0 .
$$

Proof. The essence of this result involves the inversion of Proposition 6.4 which is carried out in a manner analogous to the proof of Proposition 4.4.

Of the four coefficients $\Gamma_{n}, \Phi_{n}, \Psi_{n}, \Xi_{n}$, only two are independent because of the following relations.

Proposition 6.7. The deformation coefficients satisfy the linear identity

$$
\Psi_{n}=-\frac{a_{n}}{a_{n-1}} \Phi_{n-1}, \quad n \geq 1
$$

and the trace identity

$$
\Delta v\left(\Gamma_{n}+\Xi_{n}\right)=2 H_{n}\left[\frac{R+\Delta v S}{a_{n}\left(v_{-}\right)}-\frac{R-\Delta v S}{a_{n}\left(v_{+}\right)}\right], \quad n \geq 0 .
$$

Proof. The first relation follows by comparison of (6.24) with (6.23) and setting $H_{n} / H_{n-1}=a_{n} / a_{n-1}$. The second identity is easily seen from the expressions (6.21) and (6.22) and has already been observed in the conclusions of the workings for the proof of Proposition 6.4. 
The initial values of the deformation coefficients are

$$
\begin{aligned}
& \text { (6.40) } \quad R_{0}=-\frac{1}{2} H_{0}\left[\gamma_{0}\left(v_{-}\right)+\gamma_{0}\left(v_{+}\right)\right]\left(\frac{R+\Delta v S}{a_{0}\left(v_{-}\right) \gamma_{0}\left(v_{-}\right)}+\frac{R-\Delta v S}{a_{0}\left(v_{+}\right) \gamma_{0}\left(v_{+}\right)}\right), \\
& \text {(6.41) } \Gamma_{0}=\frac{1}{\Delta v} H_{0}\left[\gamma_{0}\left(v_{-}\right)-\gamma_{0}\left(v_{+}\right)\right]\left(\frac{R+\Delta v S}{a_{0}\left(v_{-}\right) \gamma_{0}\left(v_{-}\right)}+\frac{R-\Delta v S}{a_{0}\left(v_{+}\right) \gamma_{0}\left(v_{+}\right)}\right), \\
& (6.42) \quad \Xi_{0}=\frac{1}{\Delta v} H_{0}\left[\gamma_{0}\left(v_{-}\right)+\gamma_{0}\left(v_{+}\right)\right]\left(\frac{R+\Delta v S}{a_{0}\left(v_{-}\right) \gamma_{0}\left(v_{-}\right)}-\frac{R-\Delta v S}{a_{0}\left(v_{+}\right) \gamma_{0}\left(v_{+}\right)}\right), \\
& (6.43) \quad \Phi_{-1}=0, \quad \Phi_{0}=-2 H_{0} \gamma_{0}\left(v_{-}\right) \gamma_{0}\left(v_{+}\right) T \\
& \text { (6.44) } \quad \Psi_{0}=0 .
\end{aligned}
$$

Compatibility between the matrix recurrence relation and the deformation divided-difference equation implies the next result.

Proposition 6.8. The recurrence matrix and the deformation matrix satisfy

$$
\begin{aligned}
& B_{n+1} \cdot \mathbb{M}_{u} K_{n}-\mathbb{M}_{u} K_{n} \cdot B_{n} \\
& \quad=\mathbb{D}_{u} K_{n}-\frac{1}{4} \Delta v^{2} B_{n+1} \cdot \mathbb{D}_{u} K_{n} \cdot B_{n}, \quad n \geq 0,
\end{aligned}
$$

or equivalently

$$
\begin{aligned}
& K_{n}\left(; v_{+}\right)\left(1-\frac{1}{2} \Delta v B_{n}\right)^{-1}\left(1+\frac{1}{2} \Delta v B_{n}\right) \\
& \quad=\left(1-\frac{1}{2} \Delta v B_{n+1}\right)^{-1}\left(1+\frac{1}{2} \Delta v B_{n+1}\right) K_{n}\left(; v_{-}\right), \quad n \geq 0 .
\end{aligned}
$$

Proof. The first form of the compatibility relation is deduced by comparing

$$
\begin{aligned}
\mathbb{D}_{u} Y_{n+1} & =B_{n+1} \mathbb{M}_{u} Y_{n+1} \\
& =B_{n+1} \mathbb{M}_{u}\left(K_{n} Y_{n}\right) \\
& =B_{n+1}\left(\mathbb{M}_{u} K_{n} \cdot \mathbb{M}_{u} Y_{n}+\frac{1}{4} \Delta v^{2} \mathbb{D}_{u} K_{n} \cdot \mathbb{D}_{u} Y_{n}\right) \\
& =B_{n+1}\left(\mathbb{M}_{u} K_{n}+\frac{1}{4} \Delta v^{2} \mathbb{D}_{u} K_{n} \cdot B_{n}\right) \mathbb{M}_{u} Y_{n}
\end{aligned}
$$

and

$$
\begin{aligned}
\mathbb{D}_{u} Y_{n+1} & =\mathbb{D}_{u} K_{n} Y_{n}=\left(\mathbb{D}_{u} K_{n} \cdot \mathbb{M}_{u} Y_{n}+\mathbb{M}_{u} K_{n} \cdot \mathbb{D}_{u} Y_{n}\right) \\
& =\left(\mathbb{D}_{u} K_{n}+\mathbb{M}_{u} K_{n} \cdot B_{n}\right) \mathbb{M}_{u} Y_{n}
\end{aligned}
$$


The second form is found by computing $Y_{n+1}\left(x ; v_{+}\right)$in terms of $Y_{n}\left(x ; v_{-}\right)$ in the two possible ways corresponding to the orders of the operations $n \mapsto$ $n+1$ and $v_{-} \mapsto v_{+}$.

Compatibility of the three-term recurrence relation and the deformation divided-difference equation implies the following result, which can be found by considering the representations of the deformation coefficients given in Proposition 6.4.

Proposition 6.9. The deformation coefficients $R_{n}, \Gamma_{n}, \Phi_{n}$ satisfy recurrence relations in $n$,

$$
\begin{aligned}
& \frac{a_{n+1}\left(v_{-}\right)}{H_{n+1}}\left(-2 R_{n+1}+\Delta v \Gamma_{n+1}\right)+\frac{a_{n}\left(v_{-}\right)}{H_{n}}\left(2 R_{n}+\Delta v \Gamma_{n}\right) \\
& =-\left[x-b_{n}\left(v_{-}\right)\right] \frac{\Delta v}{H_{n}} \Phi_{n} \\
& \quad+2 a_{n}\left(v_{-}\right)\left(\frac{R+\Delta v S}{a_{n}\left(v_{-}\right)}-\frac{R-\Delta v S}{a_{n}\left(v_{+}\right)}\right), \quad n \geq 0, \\
& \frac{a_{n+1}\left(v_{+}\right)}{H_{n+1}}\left(2 R_{n+1}+\Delta v \Gamma_{n+1}\right)+\frac{a_{n}\left(v_{+}\right)}{H_{n}}\left(-2 R_{n}+\Delta v \Gamma_{n}\right) \\
& =-\left[x-b_{n}\left(v_{+}\right)\right] \frac{\Delta v}{H_{n}} \Phi_{n} \\
& \quad+2 a_{n}\left(v_{+}\right)\left(\frac{R+\Delta v S}{a_{n}\left(v_{-}\right)}-\frac{R-\Delta v S}{a_{n}\left(v_{+}\right)}\right), \quad n \geq 0 .
\end{aligned}
$$

Recurrences (6.48) and (6.49) are the analogues of combinations of (4.43) and (4.44).

Proof. The simplest proof of these relations is the one employing the definitions (6.20), (6.21), and (6.23) of Proposition 6.4. Taking the first relation, we deduce

$$
\begin{aligned}
& \frac{a_{n+1}\left(v_{-}\right)}{H_{n+1}}\left(-2 R_{n+1}+\Delta v \Gamma_{n+1}\right) \\
& \quad+\frac{a_{n}\left(v_{-}\right)}{H_{n}}\left(2 R_{n}+\Delta v \Gamma_{n}\right)+\left[x-b_{n}\left(v_{-}\right)\right] \frac{\Delta v}{H_{n}} \Phi_{n} \\
& =2(R+\Delta v S)\left[1-p_{n}\left(; v_{+}\right)\left(a_{n+1}\left(v_{-}\right) q_{n+1}\left(; v_{-}\right)\right.\right. \\
& \left.\left.\quad+b_{n}\left(v_{-}\right) q_{n}\left(v_{-}\right)+a_{n}\left(v_{-}\right) q_{n-1}\left(v_{-}\right)-x q_{n}\left(v_{-}\right)\right)\right]
\end{aligned}
$$




$$
\begin{aligned}
& -2(R-\Delta v S)\left[\frac{a_{n}\left(v_{-}\right)}{a_{n}\left(v_{+}\right)}-q_{n}\left(; v_{+}\right)\left(a_{n+1}\left(v_{-}\right) p_{n+1}\left(; v_{-}\right)\right.\right. \\
& \left.\left.+b_{n}\left(v_{-}\right) p_{n}\left(v_{-}\right)+a_{n}\left(v_{-}\right) p_{n-1}\left(v_{-}\right)-x p_{n}\left(v_{-}\right)\right)\right] \\
= & 2 a_{n}\left(v_{-}\right)\left(\frac{R+\Delta v S}{a_{n}\left(v_{-}\right)}-\frac{R-\Delta v S}{a_{n}\left(v_{+}\right)}\right)
\end{aligned}
$$

where essential use of the three-term recurrence relations (3.5) and (3.22) has been made. The second relation can be found in an identical manner.

We find bilinear identities for the deformation matrix analogous to those of Proposition 4.7.

Proposition 6.10. The deformation coefficients satisfy the bilinear or determinantal identity

$$
\begin{aligned}
R_{n}^{2} & +\frac{1}{4} \Delta v^{2}\left[\Gamma_{n} \Xi_{n}-\Phi_{n} \Psi_{n}\right] \\
& =-H_{n} R_{n}\left[\frac{R+\Delta v S}{a_{n}\left(v_{-}\right)}+\frac{R-\Delta v S}{a_{n}\left(v_{+}\right)}\right], \quad n \geq 0 .
\end{aligned}
$$

Proof. A direct method of proof is possible substituting the expressions for the deformation coefficients $R_{n}, \Gamma_{n}, \Xi_{n}, \Phi_{n}, \Psi_{n}$ in terms of products of polynomials and associated functions as given by (6.20)-(6.24) into the lefthand side of (6.50). After expansion and considerable cancellation, we recognize the form for the right-hand side.

From the above result, we can deduce matrix identities analogous to Corollary 4.3.

COROLLARY 6.2. The matrix factor appearing in (6.46) has the determinant evaluation

$$
\operatorname{det}\left(1 \pm \frac{1}{2} \Delta v B_{n}\right)=-\frac{2 H_{n}(R \mp \Delta v S)}{R_{n} a_{n}\left(v_{ \pm}\right)}, \quad n \geq 0,
$$

and its inverse is

$$
\begin{aligned}
(1 & \left. \pm \frac{1}{2} \Delta v B_{n}\right)^{-1} \\
& =-\frac{a_{n}\left(v_{ \pm}\right)}{2 H_{n}(R \mp \Delta v S)}\left(\begin{array}{cc}
R_{n} \pm \frac{1}{2} \Delta v \Xi_{n} & \mp \frac{1}{2} \Delta v \Phi_{n} \\
\mp \frac{1}{2} \Delta v \Psi_{n} & R_{n} \pm \frac{1}{2} \Delta v \Gamma_{n}
\end{array}\right), \quad n \geq 0 .
\end{aligned}
$$


Proof. We note from the matrix formula for $B_{n}(6.37)$ that

$$
\begin{aligned}
\operatorname{det}\left(1 \pm \frac{1}{2} \Delta v B_{n}\right) & =\frac{1}{R_{n}^{2}}\left\{R_{n}^{2}+\frac{1}{4} \Delta v^{2}\left[\Gamma_{n} \Xi_{n}-\Phi_{n} \Psi_{n}\right] \pm \frac{1}{2} \Delta v R_{n}\left(\Gamma_{n}+\Xi_{n}\right)\right\} \\
& =-\frac{2 H_{n}}{R_{n}} \frac{R \mp \Delta v S}{a_{n}\left(v_{ \pm}\right)}
\end{aligned}
$$

where we have used (6.50) and (6.39) in the last step. The inversion formula follows from this last result and the standard formula for an inverse.

Consequent to the results of Corollary 6.2, we have the following expression for the matrix product appearing in (6.46).

COROLlary 6.3. The matrix product (6.46) has the evaluation

$$
\begin{aligned}
(1- & \left.\frac{1}{2} \Delta v B_{n}\right)^{-1}\left(1+\frac{1}{2} \Delta v B_{n}\right) \\
= & \frac{a_{n}\left(v_{-}\right)}{2 H_{n}(R+\Delta v S)} \\
& \times\left(\begin{array}{cc}
2 R_{n}+2 H_{n} \frac{R-\Delta v S}{a_{n}\left(v_{+}\right)}+\Delta v \Gamma_{n} & \Delta v \Phi_{n} \\
\Delta v \Psi_{n} & 2 R_{n}+2 H_{n} \frac{R+\Delta v S}{a_{n}\left(v_{-}\right)}-\Delta v \Gamma_{n}
\end{array}\right), \\
n \geq & 0, \quad-a_{n}\left(v_{-}\right)\left(\begin{array}{c}
p_{n}\left(; v_{+}\right) \\
p_{n-1}\left(; v_{+}\right)
\end{array}\right) \otimes\left(q_{n-1}\left(; v_{-}\right), \quad-q_{n}\left(; v_{-}\right)\right) \\
& +a_{n}\left(v_{-}\right) \frac{R-\Delta v S}{R+\Delta v S}\left(\begin{array}{c}
q_{n}\left(; v_{+}\right) \\
q_{n-1}\left(; v_{+}\right)
\end{array}\right) \otimes\left(p_{n-1}\left(; v_{-}\right), \quad-p_{n}\left(; v_{-}\right)\right)
\end{aligned}
$$

Proof. Using the inverse (6.52), we form the matrix product and employ (6.50) to simplify the diagonal elements, with the result given by (6.53).

This result also motivates the following definitions

$$
\begin{aligned}
& \mathfrak{R}_{+}:=2 R_{n}+2 H_{n} \frac{R-\Delta v S}{a_{n}\left(v_{+}\right)}+\Delta v \Gamma_{n}, \\
& \mathfrak{R}_{-}:=2 R_{n}+2 H_{n} \frac{R+\Delta v S}{a_{n}\left(v_{-}\right)}-\Delta v \Gamma_{n}, \\
& \mathfrak{P}_{+}:=-\Delta v \Phi_{n}, \quad \mathfrak{P}_{-}:=\Delta v \Psi_{n},
\end{aligned}
$$


valid for $n \geq 1$, and for $n=0$ we have

$$
\begin{aligned}
& \mathfrak{R}_{+}(n=0):=-2 \frac{H_{0}}{a_{0}\left(v_{-}\right)} \frac{\gamma_{0}\left(v_{+}\right)}{\gamma_{0}\left(v_{-}\right)}(R+\Delta v S), \\
& \mathfrak{R}_{-}(n=0):=-2 \frac{H_{0}}{a_{0}\left(v_{+}\right)} \frac{\gamma_{0}\left(v_{-}\right)}{\gamma_{0}\left(v_{+}\right)}(R-\Delta v S), \\
& \mathfrak{P}_{+}(n=0):=2 \Delta v H_{0} \gamma_{0}\left(v_{+}\right) \gamma_{0}\left(v_{-}\right) T, \quad \mathfrak{P}_{-}(n=0):=0,
\end{aligned}
$$

together with

$$
B_{n}^{*}:=\left(\begin{array}{cc}
\mathfrak{R}_{+} & -\mathfrak{P}_{+} \\
\mathfrak{P}_{-} & \mathfrak{R}_{-}
\end{array}\right) .
$$

In these variables the deformation bi-linear relation (6.50) takes the form

$$
\operatorname{det} B_{n}^{*}=\mathfrak{R}_{+} \mathfrak{R}_{-}+\mathfrak{P}_{+} \mathfrak{P}_{-}=\frac{4 H_{n}^{2}}{a_{n}\left(v_{+}\right) a_{n}\left(v_{-}\right)}\left(R^{2}-\Delta v^{2} S^{2}\right) .
$$

It is of advantage to write out recurrence-deformation compatibility equations in terms of $\mathfrak{R}_{ \pm, n}, \mathfrak{P}_{ \pm, n}$, where again we append a subscript to indicate the dependence on the index $n$. We will henceforth denote variables evaluated at the advanced and retarded deformation variable by $\hat{a}_{n}=a_{n}\left(v_{+}\right)$, $\check{a}_{n}=a_{n}\left(v_{-}\right)$.

Corollary 6.4. Solving (6.46) for $B_{n+1}^{*}$, we deduce

$$
\begin{aligned}
& \frac{H_{n}}{H_{n+1}} \hat{a}_{n+1} \check{a}_{n+1} \mathfrak{P}_{+, n+1} \\
& =-\check{a}_{n}\left(x-\hat{b}_{n}\right) \mathfrak{R}_{+, n}+\hat{a}_{n}\left(x-\check{b}_{n}\right) \mathfrak{R}_{-, n} \\
& \quad+\left(x-\hat{b}_{n}\right)\left(x-\check{b}_{n}\right) \mathfrak{P}_{+, n}+\hat{a}_{n} \check{a}_{n} \mathfrak{P}_{-, n},
\end{aligned}
$$

$$
\frac{H_{n}}{H_{n+1}} \mathfrak{P}_{-, n+1}=\mathfrak{P}_{+, n} \text {, }
$$

$$
\frac{H_{n}}{H_{n+1}} \mathfrak{R}_{+, n+1}=\frac{\hat{a}_{n}}{\hat{a}_{n+1}} \mathfrak{R}_{-, n}+\frac{x-\hat{b}_{n}}{\hat{a}_{n+1}} \mathfrak{P}_{+, n},
$$$$
\frac{H_{n}}{H_{n+1}} \mathfrak{R}_{-, n+1}=\frac{\check{a}_{n}}{\check{a}_{n+1}} \mathfrak{R}_{+, n}-\frac{x-\check{b}_{n}}{\check{a}_{n+1}} \mathfrak{P}_{+, n} .
$$

The compatibility relation between the spectral and deformation divideddifference equations takes the following form. 
Proposition 6.11. The spectral matrix $A_{n}(x ; u)$ and the deformation matrix $B_{n}(x ; u)$ satisfy the $\mathbb{D}$-Schlesinger equation

$$
\begin{aligned}
\mathbb{D}_{u} A_{n} & -\mathbb{D}_{x} B_{n}+\mathbb{M}_{u} A_{n} \cdot \mathbb{M}_{x} B_{n}-\mathbb{M}_{x} B_{n} \cdot \mathbb{M}_{u} A_{n} \\
= & \frac{1}{4} \Delta v^{2} \mathbb{M}_{x} B_{n} \cdot \mathbb{D}_{u} A_{n} \cdot\left(1-\frac{1}{16} \Delta y^{2} \Delta v^{2} \mathbb{D}_{x} B_{n} \cdot \mathbb{D}_{u} A_{n}\right)^{-1} \\
& \times\left(\mathbb{M}_{x} B_{n}+\frac{1}{4} \Delta y^{2} \mathbb{D}_{x} B_{n} \cdot \mathbb{M}_{u} A_{n}\right) \\
& -\frac{1}{4} \Delta y^{2} \mathbb{M}_{u} A_{n} \cdot \mathbb{D}_{x} B_{n} \cdot\left(1-\frac{1}{16} \Delta y^{2} \Delta v^{2} \mathbb{D}_{u} A_{n} \cdot \mathbb{D}_{x} B_{n}\right)^{-1} \\
& \times\left(\mathbb{M}_{u} A_{n}+\frac{1}{4} \Delta v^{2} \mathbb{D}_{u} A_{n} \cdot \mathbb{M}_{x} B_{n}\right)
\end{aligned}
$$

or the equivalent form

$$
\begin{aligned}
(1- & \left.\frac{1}{2} \Delta y A_{n}\left(; v_{+}\right)\right)^{-1}\left(1+\frac{1}{2} \Delta y A_{n}\left(; v_{+}\right)\right) \\
& \times\left(1-\frac{1}{2} \Delta v B_{n}\left(y_{-} ;\right)\right)^{-1}\left(1+\frac{1}{2} \Delta v B_{n}\left(y_{-} ;\right)\right) \\
= & \left(1-\frac{1}{2} \Delta v B_{n}\left(y_{+} ;\right)\right)^{-1}\left(1+\frac{1}{2} \Delta v B_{n}\left(y_{+} ;\right)\right) \\
& \times\left(1-\frac{1}{2} \Delta y A_{n}\left(; v_{-}\right)\right)^{-1}\left(1+\frac{1}{2} \Delta y A_{n}\left(; v_{-}\right)\right) .
\end{aligned}
$$

Proof. The first form follows from $\mathbb{D}_{x} \mathbb{D}_{u} Y_{n}=\mathbb{D}_{u} \mathbb{D}_{x} Y_{n}$ and the Leibniz formulas of (2.10) and (2.11), from which we deduce

$$
\begin{aligned}
& {\left[1-\frac{1}{16} \Delta y^{2} \Delta v^{2} \mathbb{D}_{u} A_{n} \cdot \mathbb{D}_{x} B_{n}\right] \mathbb{D}_{x} \mathbb{M}_{u} Y} \\
& =\left[\mathbb{M}_{u} A_{n}+\frac{1}{4} \Delta v^{2} \mathbb{D}_{u} A_{n} \cdot \mathbb{M}_{x} B_{n}\right] \mathbb{M}_{u} \mathbb{M}_{x} Y \\
& {\left[1-\frac{1}{16} \Delta y^{2} \Delta v^{2} \mathbb{D}_{x} B_{n} \cdot \mathbb{D}_{u} A_{n}\right] \mathbb{D}_{u} \mathbb{M}_{x} Y} \\
& \quad=\left[\mathbb{M}_{x} B_{n}+\frac{1}{4} \Delta y^{2} \mathbb{D}_{x} B_{n} \cdot \mathbb{M}_{u} A_{n}\right] \mathbb{M}_{x} \mathbb{M}_{u} Y .
\end{aligned}
$$

Using equivalent forms of the spectral and deformation divided-difference equations (4.35) and (6.37), we see that 


$$
\begin{aligned}
Y\left(y_{+} ; v_{+}\right)= & \left(1-\frac{1}{2} \Delta y A_{n}\left(; v_{+}\right)\right)^{-1}\left(1+\frac{1}{2} \Delta y A_{n}\left(; v_{+}\right)\right) Y\left(y_{-} ; v_{+}\right) \\
= & \left(1-\frac{1}{2} \Delta y A_{n}\left(; v_{+}\right)\right)^{-1}\left(1+\frac{1}{2} \Delta y A_{n}\left(; v_{+}\right)\right) \\
& \times\left(1-\frac{1}{2} \Delta v B_{n}\left(y_{-} ;\right)\right)^{-1}\left(1+\frac{1}{2} \Delta v B_{n}\left(y_{-} ;\right)\right) Y\left(y_{-} ; v_{-}\right)
\end{aligned}
$$

whereas

$$
\begin{aligned}
Y\left(y_{+} ; v_{+}\right)= & \left(1-\frac{1}{2} \Delta v B_{n}\left(y_{+} ;\right)\right)^{-1}\left(1+\frac{1}{2} \Delta v B_{n}\left(y_{+} ;\right)\right) Y\left(y_{+} ; v_{-}\right) \\
= & \left(1-\frac{1}{2} \Delta v B_{n}\left(y_{+} ;\right)\right)^{-1}\left(1+\frac{1}{2} \Delta v B_{n}\left(y_{+} ;\right)\right) \\
& \times\left(1-\frac{1}{2} \Delta y A_{n}\left(; v_{-}\right)\right)^{-1}\left(1+\frac{1}{2} \Delta y A_{n}\left(; v_{-}\right)\right) Y\left(y_{-} ; v_{-}\right) .
\end{aligned}
$$

This gives us the form (6.67).

REMARK 6.2. Let us denote the two fixed points of the $x$-lattice by $x_{L}$ and $x_{R}$. By analogy with the linear lattices, let us conjecture the existence of fundamental solutions to (4.35) about $x=x_{L}, x_{R}$ which we denote by $Y_{L}, Y_{R}$, respectively. Furthermore, let us define the connection matrix

$$
P(x ; u):=\left(Y_{R}(x ; u)\right)^{-1} Y_{L}(x ; u) .
$$

From (4.35) it is clear that $P$ is a $\mathbb{D}$-constant function with respect to $x$, that is to say,

$$
P\left(y_{+} ; u\right)=P\left(y_{-} ; u\right) .
$$

In addition, it is clear from (6.37) that this type of deformation is also a connection preserving deformation in the sense that

$$
P\left(x ; v_{+}\right)=P\left(x ; v_{-}\right) .
$$

The compatibility relation (6.67) $\chi B_{n}^{*}\left(y_{+} ; u\right) A_{n}^{*}\left(x ; v_{-}\right)=A_{n}^{*}\left(x ; v_{+}\right) B_{n}^{*}\left(y_{-} ;\right.$ $u$ ) may be written componentwise in the new variables in the more practical form as

$$
\begin{aligned}
\chi & {\left[\mathfrak{W}_{+}\left(x ; v_{-}\right) \mathfrak{R}_{+}\left(y_{+} ; u\right)-\mathfrak{T}_{-}\left(x ; v_{-}\right) \mathfrak{P}_{+}\left(y_{+} ; u\right)\right] } \\
& =\mathfrak{W}_{+}\left(x ; v_{+}\right) \mathfrak{R}_{+}\left(y_{-} ; u\right)-\mathfrak{T}_{+}\left(x ; v_{+}\right) \mathfrak{P}_{-}\left(y_{-} ; u\right),
\end{aligned}
$$




$$
\begin{aligned}
\chi & {\left[\mathfrak{T}_{+}\left(x ; v_{-}\right) \mathfrak{R}_{+}\left(y_{+} ; u\right)+\mathfrak{W}_{-}\left(x ; v_{-}\right) \mathfrak{P}_{+}\left(y_{+} ; u\right)\right] } \\
= & \mathfrak{T}_{+}\left(x ; v_{+}\right) \mathfrak{R}_{-}\left(y_{-} ; u\right)+\mathfrak{W}_{+}\left(x ; v_{+}\right) \mathfrak{P}_{+}\left(y_{-} ; u\right), \\
\chi & {\left[\mathfrak{T}_{-}\left(x ; v_{-}\right) \mathfrak{R}_{-}\left(y_{+} ; u\right)+\mathfrak{W}_{+}\left(x ; v_{-}\right) \mathfrak{P}_{-}\left(y_{+} ; u\right)\right] } \\
& =\mathfrak{T}_{-}\left(x ; v_{+}\right) \mathfrak{R}_{+}\left(y_{-} ; u\right)+\mathfrak{W}_{-}\left(x ; v_{+}\right) \mathfrak{P}_{-}\left(y_{-} ; u\right), \\
\chi & {\left[\mathfrak{W}_{-}\left(x ; v_{-}\right) \mathfrak{R}_{-}\left(y_{+} ; u\right)-\mathfrak{T}_{+}\left(x ; v_{-}\right) \mathfrak{P}_{-}\left(y_{+} ; u\right)\right] } \\
& =\mathfrak{W}_{-}\left(x ; v_{+}\right) \mathfrak{R}_{-}\left(y_{-} ; u\right)-\mathfrak{T}_{-}\left(x ; v_{+}\right) \mathfrak{P}_{+}\left(y_{-} ; u\right),
\end{aligned}
$$

where $\chi$ is defined by (6.17). The real content of (6.70)-(6.73) lies in the case $n \geq 1$ as the $n=0$ evaluations of (6.72) are trivially satisfied, whereas that of (6.70) and (6.73) are identically satisfied due to the definition (6.17), and (6.71) is equivalent to the consistency identity (6.16).

Having assembled all the ingredients of our theory, we have to draw them together and perform three tasks. The first is to parameterize the spectral coefficients in a minimal way consistent with the constraints of the spectral structures, namely, (4.46). The second task is to close this system, that is to say, relate the deformation coefficients to the spectral coefficients, preferably with the parameterization found in the first step. The third task is to derive the dynamical equations for this parameterization with respect to the deformation variable. There are two avenues of approach to the problem of extracting useful information from the above compatibility relations (6.70)(6.73). One way is to clear the denominators on both sides and resolve the resulting expressions in terms of $\bigoplus_{k \geq 0} x^{k}+\Delta y \bigoplus_{k \geq 0} x^{k}$. This is useful for certain results, as we will see in the application. The other method is to work with the evaluation of the spectral and deformation coefficients at certain key ordinates and construct a parameterization based upon these variables. A fundamental and crucial role will be played by the zeros of the polynomials $W^{2}-\Delta y^{2} V^{2}, \Theta_{n}$ and $\chi$ as these ordinates, however these cannot be interpreted as singularities in the spectral plane.

REMARK 6.3. The compatibility relations (6.70)-(6.73) are satisfied identically at the common zeros of $\left(W^{2}-\Delta y^{2} V^{2}\right)\left(\tilde{x} ; v_{ \pm}\right)$in the sense that both sides of the relations are identically zero, that is to say, at those fixed zeros independent of $u$. In addition, one can show that the left-hand side of the bilinear relation (6.61) vanishes at the movable zeros in either of the two above cases. This implies that when $\left(W^{2}-\Delta y^{2} V^{2}\right)\left(\tilde{x} ; v_{ \pm}\right)=0$, then $\left(R^{2}-\Delta v^{2} S^{2}\right)\left(\tilde{y}_{ \pm} ; u\right)=0$. 


\section{$\S 7$. The $M=3, L=1$ cases and deformation of the Askey-Wilson OPS}

The previous sections treated the $\mathbb{D}$-semiclassical orthogonal polynomial system with general divided-difference operators $\mathbb{D}, \mathbb{M}$ and arbitrary degrees $M, L$ for the spectral and deformation coefficients, respectively. Here we apply the foregoing theory to the symmetrized form of the $q$-quadratic lattice in the elliptic subcase, which singles out the Askey-Wilson weight and the nontrivial examples of deformations of this weight. We will consider the situation of the deformation variable also on this subcase of the $q$-quadratic lattice. The deformation variable is $u=\left(t+t^{-1}\right) / 2$ with $t=e^{i \phi}$, and the analogous relations for the nodes on the deformation lattice are

$$
\begin{aligned}
v_{+}+v_{-} & =\left(q^{1 / 2}+q^{-1 / 2}\right) u, \\
v_{+}-v_{-} & =\frac{1}{2}\left(q^{1 / 2}-q^{-1 / 2}\right)\left(t-t^{-1}\right), \\
\Delta v^{2} & =\left(q^{1 / 2}-q^{-1 / 2}\right)^{2}\left(u^{2}-1\right), \\
v_{+} v_{-} & =u^{2}+\frac{1}{4}\left(q^{1 / 2}-q^{-1 / 2}\right)^{2} .
\end{aligned}
$$

\subsection{Moments and integrals}

Our starting point is the simplest extension of the $M=2$ case

$$
W \pm \Delta y V=z^{\mp 3} \prod_{j=1}^{6}\left(1-a_{j} q^{-1 / 2} z^{ \pm 1}\right),
$$

which is a minimal, natural extension of the Askey-Wilson case given by (5.7). The reader should appreciate that all of the conclusions we are going to draw will follow entirely from (7.5), so that any valid solution of the Pearson equation (4.2) for the weight with this data is as acceptable as any other. The most important consideration in selecting a solution is the support for the weight and existence of the moment data. As we will see (i.e., in Propositions 7.2 and 7.7), the only place where a specific choice for the weight enters is in determining the initial values of recurrences or divided-difference equations. The four fixed parameters $a_{1}, \ldots, a_{4}$ appear in the same form as they do in the Askey-Wilson weight $(M=2)$, and so the weight constitutes the base expression, and we seek to introduce an extra parameter and its associated deformation variable. We set $a_{5}=\alpha t$, $a_{6}=\alpha t^{-1}$, and we designate $\alpha, t$ as the deformation parameter and variable, respectively. 
REMARK 7.1. One might entertain the possibility of a weight with the spectral data

$$
W \pm \Delta y V=z^{\mp 2} \prod_{j=1}^{4}\left(1-q^{-1 / 2} a_{j} z^{ \pm 1}\right)\left(1-q^{-1 / 2} t z^{ \pm 1}\right)\left(1-q^{\alpha-1 / 2} z^{\mp 1}\right)
$$

which is an $M=3$ case on a $q$-quadratic spectral lattice, however it is $q$ linear in either of the deformation variables $t, q^{\alpha}$. This is in fact the spectral data for the weight in the integral representation of the very-well-poised ${ }_{8} W_{7}$ basic hypergeometric function (see [81, (1.13)]). This function is the classical seed solution for the $E_{7}^{(1)} q$-Painlevé system as constructed by [52].

Our first undertaking is to derive linear recurrences for the moments which will be required in the verification of key identities from Sections 4 and 6 , to make contact with the $n=0$ seed solutions of the evolution equations derived later, and for checking purposes. Through suitable choices of parameters $k, b, a$, the moment recurrences can be recast as $q$-difference equations for an integral $I_{3}\left(a_{1}, \ldots, a_{6}\right)$ which is a generalization of the Askey-Wilson integral (5.24).

Proposition 7.1 ([97]). Let $\sigma_{k}$ denote the $k$ th elementary symmetric polynomial in $a_{1}, \ldots, a_{4}$ or $a_{1}, \ldots, a_{6}$, depending on the context. The integral $I_{3}\left(a_{1}, \ldots, a_{6}\right)$ satisfies a three-term recurrence in one variable, which we take without loss of generality with respect to $a_{1}$,

$$
\begin{aligned}
0= & \prod_{j \neq 1}\left(a_{1} a_{j}-1\right) I_{3}\left(a_{1}, \ldots\right) \\
& +\left[1+q^{-1}-a_{1}\left(\sum_{j \neq 1} a_{j}-q a_{1}\right)\right. \\
& \left.+\prod_{1}^{6} a_{j}\left(a_{1} \sum_{k \neq 1}^{5} a_{k}^{-1}-q^{-1}-(q+1) a_{1}^{2}\right)\right] I_{3}\left(q a_{1}, \ldots\right) \\
& +q^{-1}\left(\prod_{1}^{6} a_{j}-1\right) I_{3}\left(q^{2} a_{1}, \ldots\right)
\end{aligned}
$$

In addition, the integral $I_{3}\left(a_{1}, \ldots, a_{6}\right)$ satisfies a three-term recurrence in two variables, taken to be with respect to $a_{5}, a_{6}$, which constitutes a pure 
recurrence in the deformation variable $u$

$$
\begin{aligned}
0= & \left(a_{5}-q a_{6}\right) \prod_{j=1}^{4}\left(1-a_{j} a_{6}\right) I_{3}\left(\ldots, q^{2} a_{5}, a_{6}\right) \\
& -\left(a_{5}-a_{6}\right)\left[(1+q)\left(1+q a_{5} a_{6} \sigma_{2}+q^{2} a_{5}^{2} a_{6}^{2} \sigma_{4}\right)\right. \\
& -\left(q a_{5}-a_{6}\right)\left(q a_{6}-a_{5}\right)\left(q+\sigma_{4}\right) \\
& \left.-q\left(a_{5}+a_{6}\right)\left(\sigma_{1}+q a_{5} a_{6} \sigma_{3}\right)\right] I_{3}\left(\ldots, q a_{5}, q a_{6}\right) \\
& +\left(q a_{5}-a_{6}\right) \prod_{j=1}^{4}\left(1-a_{j} a_{5}\right) I_{3}\left(\ldots, a_{5}, q^{2} a_{6}\right) .
\end{aligned}
$$

Proof. The first recurrence can be read off from (4.11) specialized to $k=0$, after evaluating the expansion coefficients

$$
\begin{aligned}
& \delta_{3,2}(a)=\frac{\sigma_{6}-1}{q^{5 / 2} a^{2}} \\
& \delta_{3,1}(a)=\frac{1}{q^{5 / 2} a^{2}}\left[q a\left(\sigma_{5}-\sigma_{1}\right)-(1+q)\left(1+q a^{2}\right)\left(\sigma_{6}-1\right)\right] \\
& \delta_{3,0}(a)=\frac{1}{q^{3 / 2} a^{2}}\left[\left(1+a^{2}+a^{4}\right)\left(\sigma_{6}-1\right)-\left(a+a^{3}\right)\left(\sigma_{5}-\sigma_{1}\right)+a^{2}\left(\sigma_{4}-\sigma_{2}\right)\right] .
\end{aligned}
$$

The second recurrence follows from combining the first with the general identity

$$
a_{k} I\left(q a_{j}, \ldots, a_{k}\right)-a_{j} I\left(a_{j}, \ldots, q a_{k}\right)=\left(a_{k}-a_{j}\right)\left(1-a_{j} a_{k}\right) I\left(a_{j}, \ldots, a_{k}\right),
$$

which applies to any integral with products of $\phi_{\infty}\left(x ; a_{j}\right) \phi_{\infty}\left(x ; a_{k}\right)$ in the denominator of the integrand and any distinct pair $a_{j} \neq a_{k}$.

REMARK 7.2. A particular solution of (7.8) which serves as a concrete example of a moment sequence is one taken from [97, Proposition 3.4]

$$
\begin{aligned}
m_{0,0}(t)= & t^{1 / 2}\left(q \alpha t, \alpha^{-1} t^{-1}, q^{1 / 2} \alpha t, q^{1 / 2} \alpha^{-1} t^{-1} ; q\right)_{\infty} \\
& \times \frac{\left(a_{1}^{-1} \sigma_{4} \alpha t, a_{2}^{-1} \sigma_{4} \alpha t, a_{3}^{-1} \sigma_{4} \alpha t, a_{4}^{-1} \sigma_{4} \alpha t ; q\right)_{\infty}}{\left(a_{1} \alpha t, a_{2} \alpha t, a_{3} \alpha t, a_{4} \alpha t, t^{-2}, \sigma_{4} \alpha^{2} t^{2} ; q\right)_{\infty}} \\
& \times{ }_{8} W_{7}\left(q^{-1} \sigma_{4} \alpha^{2} t^{2} ; q^{-1} \sigma_{4} \alpha^{2}, a_{1} \alpha t, a_{2} \alpha t, a_{3} \alpha t, a_{4} \alpha t ; q \alpha^{-2}\right) \\
& +\left(t \mapsto t^{-1}\right)
\end{aligned}
$$

although this is not a general solution of the $q$-difference equation, given subsequently as (7.78). 
Henceforth we denote $\tilde{\sigma}_{j}$ as the $j$ th elementary symmetric function of the parameters $q^{-1 / 2} a_{1}, \ldots, q^{-1 / 2} a_{6}$. If $\sigma_{k}$ denotes the $k$ th elementary symmetric function of $a_{1}, a_{2}, a_{3}, a_{4}$, then

$$
\begin{aligned}
& \tilde{\sigma}_{1}=q^{-1 / 2}\left(\sigma_{1}+2 \alpha u\right), \\
& \tilde{\sigma}_{2}=q^{-1}\left(\sigma_{2}+\alpha^{2}+2 \alpha \sigma_{1} u\right), \\
& \tilde{\sigma}_{3}=q^{-3 / 2}\left(\sigma_{3}+\alpha^{2} \sigma_{1}+2 \alpha \sigma_{2} u\right), \\
& \tilde{\sigma}_{4}=q^{-2}\left(\sigma_{4}+\alpha^{2} \sigma_{2}+2 \alpha \sigma_{3} u\right), \\
& \tilde{\sigma}_{5}=q^{-5 / 2}\left(\alpha^{2} \sigma_{3}+2 \alpha \sigma_{4} u\right), \\
& \tilde{\sigma}_{6}=q^{-3} \alpha^{2} \sigma_{4} .
\end{aligned}
$$

We will find it advantageous to define two "analogues" of the integers or half-integers $s$ by $[s]:=q^{s} \tilde{\sigma}_{6}-q^{-s}$ and $\{s\}:=q^{s} \tilde{\sigma}_{6}+q^{-s}$. For convenience, we employ the notations $w_{ \pm}$for the evaluations $w_{ \pm} \equiv W( \pm 1)= \pm 1-\tilde{\sigma}_{1} \pm$ $\tilde{\sigma}_{2}-\tilde{\sigma}_{3} \pm \tilde{\sigma}_{4}-\tilde{\sigma}_{5} \pm \tilde{\sigma}_{6}$. We also require the coordinates $x_{j}$ and $\tilde{x}_{j}$ defined in terms of the parameters by $x_{j}=\frac{1}{2}\left(a_{j}+a_{j}^{-1}\right)$ and $\tilde{x}_{j}=\frac{1}{2}\left(q^{-1 / 2} a_{j}+q^{1 / 2} a_{j}^{-1}\right)$. Finally, let us define the polynomial $w(z):=\prod_{j=1}^{4}\left(1-q^{-1 / 2} a_{j} z\right)$, which is not to be confused with the weight $w(x ; u)$.

Definition 7.1. Note that we have six free parameters at our disposal, $a_{1}, \ldots, a_{4}, \alpha \in \mathbb{C}, n \in \mathbb{Z}_{\geq 0}$ and one variable $t$ subject henceforth to the following generic conditions:

(i) $q \neq 1$,

(ii) $\left|q^{-1 / 2} a_{j}\right| \neq 1$ so that $\tilde{x}_{j} \notin(-1,1)$ for $j=1, \ldots, 4$,

(iii) $\left|q^{-1 / 2} \alpha\right| \neq 1$ so that $q^{-1 / 2} \alpha+q^{1 / 2} \alpha^{-1} \notin(-1,1)$,

(iv) $\alpha \neq 0, \pm q^{1 / 2}$,

(v) $t \neq \pm 1$,

(vi) $[n],\left[n+\frac{1}{2}\right] \neq 0$.

Situations where one or more of the above conditions are violated have to be treated separately, which we refrain from doing here.

As a consequence of (7.5), we have

$$
\begin{aligned}
W(x)= & 4\left(1+\tilde{\sigma}_{6}\right) x^{3}-2\left(\tilde{\sigma}_{1}+\tilde{\sigma}_{5}\right) x^{2} \\
& +\left(\tilde{\sigma}_{2}+\tilde{\sigma}_{4}-3-3 \tilde{\sigma}_{6}\right) x+\tilde{\sigma}_{1}-\tilde{\sigma}_{3}+\tilde{\sigma}_{5},
\end{aligned}
$$




$$
\begin{aligned}
V(x)= & \frac{1}{q^{1 / 2}-q^{-1 / 2}}\left[-4\left(1-\tilde{\sigma}_{6}\right) x^{2}+2\left(\tilde{\sigma}_{1}-\tilde{\sigma}_{5}\right) x\right. \\
& \left.+1-\tilde{\sigma}_{2}+\tilde{\sigma}_{4}-\tilde{\sigma}_{6}\right] .
\end{aligned}
$$

The theory detailed in Section 4 allows us to evaluate the third spectral data polynomial.

Proposition $7.2([97])$. The polynomial $U(x)$ is given by

$$
\begin{aligned}
U(x)= & \frac{4}{\left(q^{1 / 2}-q^{-1 / 2}\right)}\left[m_{0,0}\left(-2\left[\frac{1}{2}\right] x+q^{1 / 2} \tilde{\sigma}_{5}-q^{-1 / 2} \tilde{\sigma}_{1}\right)\right. \\
& \left.-[1]\left(m_{0,+}+m_{0,-}\right)\right],
\end{aligned}
$$

where $m_{0, \pm}=\int \mathbb{D} x w(x ; u) z^{ \pm}$.

Proof. We start with

$$
\Delta y U(x)=(W-\Delta y V) f\left(y_{+}\right)-(W+\Delta y V) f\left(y_{-}\right),
$$

and employ the proof in [97]. Having achieved the task of expanding the right-hand side of (7.19) in canonical basis polynomials, all we require are explicit expressions for the coefficients given below:

$$
\begin{aligned}
& \kappa_{3,2}(a)=\frac{1}{2 q^{9 / 2} a^{3}}\left[\left(1+q+q^{2}\right)\left(1+q^{2} a^{2}\right)\left(1+\sigma_{6}\right)-q^{2} a\left(\sigma_{1}+\sigma_{5}\right)\right], \\
& \kappa_{3,3}(a)=-\frac{1+\sigma_{6}}{2 q^{9 / 2} a^{3}} .
\end{aligned}
$$

The result is (7.18).

\subsection{Spectral structure}

Our next task is to construct a minimal parameterization of the spectral matrix $A_{n}(x ; u)$, which is the subject of the following proposition. We also recall the large $x$ expansions for the spectral data and coefficients of a more explicit nature which will be of importance:

$$
\begin{aligned}
W \pm \Delta y V \sim & {\left[w \pm\left(q^{1 / 2}-q^{-1 / 2}\right) v\right] x^{M}+\cdots } \\
W_{n} \sim & \frac{1}{4}\left[\left(1+q^{n}\right)\left(1+q^{-n}\right) w\right. \\
& \left.+\left(q^{1 / 2}-q^{-1 / 2}\right)\left(q^{n}-q^{-n}\right) v\right] x^{M}+\cdots,
\end{aligned}
$$




$$
\begin{aligned}
\Theta_{n} \sim & {\left[\frac{q^{n+1 / 2}-q^{-n-1 / 2}}{q^{1 / 2}-q^{-1 / 2}} w\right.} \\
& \left.+\left(q^{n+1 / 2}+q^{-n-1 / 2}\right) v\right] x^{M-1}+\cdots, \\
\Omega_{n}+V \sim & \frac{1}{2}\left[\frac{q^{n}-q^{-n}}{q^{1 / 2}-q^{-1 / 2}} w+\left(q^{n}+q^{-n}\right) v\right] x^{M-2}+\cdots,
\end{aligned}
$$

for some constants $w, v$ independent of $n, x$.

Proposition 7.3. Let one of our free variables, $\lambda_{n}(u)$, be the zero of $\Theta_{n}(x ; u)$ with respect to $x$. Also define the other independent variables for $n \geq 0$ by

$$
\begin{aligned}
& \nu_{n}(u) \equiv 2 W_{n}\left(\lambda_{n} ; u\right)-W\left(\lambda_{n} ; u\right), \\
& \mu_{n}(u) \equiv \Omega_{n}\left(\lambda_{n} ; u\right)+V\left(\lambda_{n} ; u\right),
\end{aligned}
$$

which are related by the spectral conic equation

$$
\nu_{n}^{2}-W^{2}\left(\lambda_{n}\right)=\Delta\left(\lambda_{n}^{2}-1\right)\left[\mu_{n}^{2}-V^{2}\left(\lambda_{n}\right)\right],
$$

again valid for $n \geq 0$. Let us assume that $\lambda_{n}(u) \neq \pm 1$ for all $n, u$. The spectral coefficients for the $M=3$ deformed Askey-Wilson OPS are parameterized by $\lambda_{n}$, and either $\nu_{n}$ or $\mu_{n}$, through the expressions

$$
\begin{aligned}
2 W_{n}(x ; u)-W(x ; u) & \\
= & \frac{x^{2}-1}{\lambda_{n}^{2}-1} \nu_{n}+\left(x-\lambda_{n}\right)\left[4\{n\}\left(x^{2}-1\right)\right. \\
& \left.+\frac{1}{2} w_{+} \frac{x+1}{1-\lambda_{n}}+\frac{1}{2} w_{-} \frac{x-1}{1+\lambda_{n}}\right], \\
\Omega_{n}(x & ; u)+V(x ; u) \\
= & \mu_{n}+4 \frac{[n]}{q^{1 / 2}-q^{-1 / 2}} x\left(x-\lambda_{n}\right) \\
& +\frac{16}{q^{1 / 2}-q^{-1 / 2}} \frac{\tilde{\sigma}_{6}}{[n]}\left[\frac{\tilde{\sigma}_{1} \tilde{\sigma}_{6}+\tilde{\sigma}_{5}}{4 \tilde{\sigma}_{6}}-\lambda_{n}\right]\left(x-\lambda_{n}\right) \\
& +\frac{1}{q^{1 / 2}-q^{-1 / 2}} \frac{\{n\}}{[n]}\left(x-\lambda_{n}\right) \\
& \times\left[\frac{\nu_{n}}{\lambda_{n}^{2}-1}+\frac{1}{2} w_{+} \frac{1}{1-\lambda_{n}}+\frac{1}{2} w_{-} \frac{1}{1+\lambda_{n}}\right],
\end{aligned}
$$


and

$$
\Theta_{n}(x ; u)=8 \frac{\left[n+\frac{1}{2}\right]}{q^{1 / 2}-q^{-1 / 2}}\left(x-\lambda_{n}\right),
$$

for $n \geq 0$.

Proof. First, we recall the spectral data polynomials given by (7.5), (7.16), and (7.17). The product $W^{2}-\Delta y^{2} V^{2}$ plays a significant role, and therefore we define another set of elementary symmetric polynomials by

$$
\begin{aligned}
W^{2}-\Delta y^{2} V^{2} & =C_{\infty} \prod_{j=1}^{6}\left(x-\tilde{x}_{j}\right) \\
& =C_{\infty}\left[x^{6}-e_{1} x^{5}+e_{2} x^{4}-e_{3} x^{3}+e_{4} x^{2}-e_{5} x+e_{6}\right],
\end{aligned}
$$

where $C_{\infty}=64 \tilde{\sigma}_{6}$. We parameterize the spectral coefficients in the following way:

$$
\begin{aligned}
2 W_{n}-W & =w_{3} x^{3}+w_{2} x^{2}+w_{1} x+w_{0}, \\
\Theta_{n} & =\varpi_{+}\left(x-\lambda_{n}\right), \quad \Theta_{n-1}=\varpi_{-}\left(x-\lambda_{n-1}\right), \\
\Omega_{n}+V & =v_{2} x^{2}+v_{1} x+v_{0},
\end{aligned}
$$

where the leading order coefficients in each are trivial and given by using (7.16) and (7.17) in the expansions (4.28), (4.29), and (4.30):

$$
w_{3}=4\{n\}, \quad \varpi_{+}=8 \frac{\left[n+\frac{1}{2}\right]}{q^{1 / 2}-q^{-1 / 2}}, \quad v_{2}=4 \frac{[n]}{q^{1 / 2}-q^{-1 / 2}} .
$$

From the fundamental bilinear relation (4.46), we get a system of quadratic polynomial equalities

$$
\begin{gathered}
w_{3}^{2}-\Delta v_{2}^{2}=C_{\infty}, \\
2 w_{2} w_{3}-2 \Delta v_{1} v_{2}=-C_{\infty} e_{1}, \\
2 w_{1} w_{3}+w_{2}^{2}-\Delta\left(v_{1}^{2}-v_{2}^{2}+2 v_{0} v_{2}\right)+a_{n}^{2} \Delta \varpi_{+} \varpi_{-}=C_{\infty} e_{2}, \\
2 w_{1} w_{2}+2 w_{0} w_{3}-2 \Delta\left(v_{0} v_{1}-v_{1} v_{2}\right) \\
-a_{n}^{2} \Delta \varpi_{+} \varpi_{-}\left(\lambda_{n}+\lambda_{n-1}\right)=-C_{\infty} e_{3},
\end{gathered}
$$




$$
\begin{gathered}
w_{1}^{2}+2 w_{0} w_{2}-\Delta\left(v_{0}^{2}-v_{1}^{2}-2 v_{0} v_{2}\right) \\
+a_{n}^{2} \Delta \varpi_{+} \varpi_{-}\left(\lambda_{n} \lambda_{n-1}-1\right)=C_{\infty} e_{4}, \\
2 w_{0} w_{1}+2 \Delta v_{0} v_{1}+a_{n}^{2} \Delta \varpi_{+} \varpi_{-}\left(\lambda_{n}+\lambda_{n-1}\right)=-C_{\infty} e_{5}, \\
w_{0}^{2}+\Delta v_{0}^{2}-a_{n}^{2} \Delta \varpi_{+} \varpi_{-} \lambda_{n} \lambda_{n-1}=C_{\infty} e_{6},
\end{gathered}
$$

where $\Delta$ is, again, defined as $\left(q^{1 / 2}-q^{-1 / 2}\right)^{2}$. Now $(7.42),(7.40),(7.38)$, and (7.36) imply $\left(w_{0}+w_{2}\right)^{2}+\left(w_{1}+w_{3}\right)^{2}=C_{\infty}\left(1+e_{2}+e_{4}+e_{6}\right)$, while (7.41), (7.39), and (7.37) imply $2\left(w_{0}+w_{2}\right)\left(w_{1}+w_{3}\right)=-C_{\infty}\left(e_{1}+e_{3}+e_{5}\right)$. Forming the sum and difference of these two later relations, we conclude that

$$
\begin{aligned}
& w_{1}+w_{3}=\frac{\epsilon W(1)+\epsilon^{\prime} W(-1)}{2}, \\
& w_{0}+w_{2}=\frac{\epsilon W(1)-\epsilon^{\prime} W(-1)}{2},
\end{aligned}
$$

where $\epsilon, \epsilon^{\prime}= \pm 1$ and are yet to be determined. Using (7.43) along with the above definition we can solve for $w_{0}, w_{2}$, which only leaves the signs $\epsilon, \epsilon^{\prime}$ unresolved. These can be fixed by requiring that the $n=0$ evaluation of $2 W_{n}-W$ precisely reproduces $W$. This is identically true for all free parameters $a_{1}, \ldots, a_{6}$ provided that $\epsilon=+1, \epsilon^{\prime}=-1$, and yields (7.46) and (7.44). The above coefficients are given by

$$
\begin{aligned}
& w_{2}=\frac{\nu_{n}}{\lambda_{n}^{2}-1}-w_{3} \lambda_{n}-\frac{1}{2} W(1) \frac{1}{\lambda_{n}-1}+\frac{1}{2} W(-1) \frac{1}{\lambda_{n}+1}, \\
& w_{1}=-w_{3}+\frac{1}{2} W(1)-\frac{1}{2} W(-1), \\
& w_{0}=-\frac{\nu_{n}}{\lambda_{n}^{2}-1}+w_{3} \lambda_{n}+\frac{1}{2} W(1) \frac{\lambda_{n}}{\lambda_{n}-1}+\frac{1}{2} W(-1) \frac{\lambda_{n}}{\lambda_{n}+1} .
\end{aligned}
$$

We observe that this condition also gives $\nu_{0}=W\left(\lambda_{0}\right)$, as it must. Through knowledge of $w_{2}$ and utilizing $(7.37)$ we can determine $v_{1}$, which is given in (7.47):

$$
\begin{aligned}
\frac{\Delta v_{2}}{w_{3}} v_{1}= & \frac{\nu_{n}}{\lambda_{n}^{2}-1}-w_{3} \lambda_{n}+\frac{1}{2} \frac{C_{\infty} e_{1}}{w_{3}} \\
& -\frac{1}{2} W(1) \frac{1}{\lambda_{n}-1}+\frac{1}{2} W(-1) \frac{1}{\lambda_{n}+1} .
\end{aligned}
$$


However to find the remaining coefficient $v_{0}$ we require $\mu_{n}$, and this result is given by (7.48):

$$
\begin{aligned}
\frac{\Delta v_{2}}{w_{3}} v_{0}= & \frac{\Delta v_{2}}{w_{3}} \mu_{n}-\frac{\lambda_{n}}{\lambda_{n}^{2}-1} \nu_{n}+\frac{C_{\infty}}{w_{3}} \lambda_{n}^{2}-\frac{1}{2} \frac{C_{\infty} e_{1}}{w_{3}} \lambda_{n} \\
& +\frac{1}{2} W(1) \frac{\lambda_{n}}{\lambda_{n}-1}-\frac{1}{2} W(-1) \frac{\lambda_{n}}{\lambda_{n}+1} .
\end{aligned}
$$

When we examine $\Omega_{n}+V$ at $n=0$ (recall that $\Omega_{0}=0$ ), we find in addition to previously found relations the equality $\mu_{0}=V\left(\lambda_{0}\right)$, again confirming our definition. Therefore, we have succeeded in relating the subleading coefficients explicitly in terms of two independent variables.

We now address the question of representations for the three-term recurrence coefficients. We will find that another set of variables, although equivalent to $\nu_{n}$ and $\mu_{n}$, will lead to the simplest forms for the relations we seek:

$$
\begin{aligned}
w_{2, n}:= & \frac{\nu_{n}}{\lambda_{n}^{2}-1}-4\{n\} \lambda_{n}-\frac{1}{2} W(1) \frac{1}{\lambda_{n}-1}+\frac{1}{2} W(-1) \frac{1}{\lambda_{n}+1}, \\
v_{0, n}:= & \left(q^{1 / 2}-q^{-1 / 2}\right) \mu_{n}-4[n] \lambda_{n}^{2} \\
& -\frac{1}{[n]} \lambda_{n}\left(\{n\} w_{2, n}+4\left(\tilde{\sigma}_{1} \tilde{\sigma}_{6}+\tilde{\sigma}_{5}\right)\right) .
\end{aligned}
$$

To achieve this outcome, we require the following recurrence relations.

Proposition 7.4. The dynamical variables $w_{2, n}(u)$ and $v_{0, n}(u)$ satisfy the following system of first-order coupled recurrence relations in $n$

$$
\begin{aligned}
w_{2, n+1}-w_{2, n}= & -4\left(q^{1 / 2}-q^{-1 / 2}\right)\left[n+\frac{1}{2}\right] \lambda_{n}, \\
v_{0, n+1}+v_{0, n}=- & \frac{\left[n+\frac{1}{2}\right]}{[n+1][n]} \\
& \times\left(2\left\{n+\frac{1}{2}\right\} \lambda_{n} w_{2, n}+8\left[n+\frac{1}{2}\right][n] \lambda_{n}^{2}\right. \\
& \left.+4\left(q^{1 / 2}+q^{-1 / 2}\right)\left(\tilde{\sigma}_{1} \tilde{\sigma}_{6}+\tilde{\sigma}_{5}\right) \lambda_{n}\right),
\end{aligned}
$$

and are valid for $n \geq 0$ and subject to the initial conditions $U\left(\lambda_{0} ; u\right)=0$, $w_{2,0}=-2\left(\tilde{\sigma}_{1}+\tilde{\sigma}_{5}\right)$, and $v_{0,0}=1-\tilde{\sigma}_{2}+\tilde{\sigma}_{4}-\tilde{\sigma}_{6}$. 
Proof. For the first recurrence, we employ our expressions (7.28) and (7.30) in (4.43) and equate the coefficients. The coefficients of $x^{3}$ and $x$ are identically satisfied whereas that of either $x^{2}$ or $x^{0}$ yields (7.51). The second recurrence follows from the examination of the $x^{0}$ terms in (4.44) and the employment of the first recurrence. We note that the terms in $x^{2}$ cancel identically, as they must.

Explicit evaluations of the three-term recurrence coefficients can be given in terms of this alternative set of variables.

Proposition 7.5. The three-term recurrence coefficients are found to be given by

$$
\begin{aligned}
16[n & \left.+\frac{1}{2}\right][n]^{2}\left[n-\frac{1}{2}\right] a_{n}^{2} \\
= & \tilde{\sigma}_{6} w_{2, n}^{2}+2\left(\tilde{\sigma}_{1} \tilde{\sigma}_{6}+\tilde{\sigma}_{5}\right)\{n\} w_{2, n}+2[n]^{3} v_{0, n} \\
& +2[n]^{2}\left(4 \tilde{\sigma}_{6}+2 \tilde{\sigma}_{4}+2 \tilde{\sigma}_{2} \tilde{\sigma}_{6}+2 \tilde{\sigma}_{1} \tilde{\sigma}_{5}\right. \\
& \left.-\{n\}\left(1+\tilde{\sigma}_{2}+\tilde{\sigma}_{4}+\tilde{\sigma}_{6}\right)+2[n]^{2}\right)+4\left(\tilde{\sigma}_{1} \tilde{\sigma}_{6}+\tilde{\sigma}_{5}\right)^{2},
\end{aligned}
$$

for $n \geq 0$ assuming $a_{0}^{2}=0$, and

$$
\begin{aligned}
{[n+1][n] b_{n}=} & -\frac{1}{4}\left\{n+\frac{1}{2}\right\} w_{2, n}-\left[n+\frac{1}{2}\right][n] \lambda_{n} \\
& -\frac{1}{2}\left(q^{1 / 2}+q^{-1 / 2}\right)\left(\tilde{\sigma}_{1} \tilde{\sigma}_{6}+\tilde{\sigma}_{5}\right),
\end{aligned}
$$

again valid for $n \geq 0$.

Proof. Using (7.38), we can solve for $a_{n}^{2}$. Observe that the right-hand side of this equation is independent of $n$ whereas individual terms on the lefthand side are. If we assume that $a_{0}^{2}=0$, then the right-hand side is equal to that of the left-hand side evaluated at $n=0$, which we know because we can express it simply in terms of the parameters. This means that $a_{n}^{2}$ is expressible as a sum of differences, and after considerable simplification we arrive at the expression (7.53). To find (7.54), we start with (4.44) and use our previous results for (7.30) and (7.29). Examining the terms in $x$, we find an expression for $b_{n}$ in terms of $\lambda_{n}, \nu_{n}$ and $\lambda_{n+1}, \nu_{n+1}$, or equivalently 
in terms of $\lambda_{n}, w_{2, n}$ and $\lambda_{n+1}, w_{2, n+1}$. Using (7.51), we can eliminate the $w_{2, n+1}$ term and after some factorization we arrive at (7.54).

We will not pursue the theory for the $n \mapsto n+1$ recurrences any further here but refer the reader to [96]. Consequently, we will drop the $n$ subscript from most variables until Section 7.4.

The foregoing parameterization of our system given in Proposition 7.3 is not useful in the investigations of the $u$ - or $t$-evolution of our system and we require an alternative construction. In conformance with this, we define the auxiliary variables $l(t)$ and $\mathfrak{z}_{ \pm}(t)$ by

$$
\begin{aligned}
\lambda & :=\frac{1}{2}\left(l+l^{-1}\right), \\
\mathfrak{z}_{ \pm} & :=\nu \pm \frac{1}{2}\left(q^{1 / 2}-q^{-1 / 2}\right)\left[l-l^{-1}\right] \mu,
\end{aligned}
$$

where, in the first case, the inversion is given by the branch whereby $l \rightarrow \infty$ when $\lambda \rightarrow \infty$, and an identical choice is made for the second case.

Proposition 7.6. The spectral matrix elements have an alternative parameterization

$$
\mathfrak{T}_{+}(z ; t)=2 a_{n}\left[n+\frac{1}{2}\right]\left(z-z^{-1}\right)(z-l)\left(1-l^{-1} z^{-1}\right),
$$

and

$$
\begin{aligned}
{[n] \mathfrak{W}_{+} } & (z ; t) \\
= & \mathfrak{z}_{+} \frac{\left(z-z^{-1}\right)\left(z-l^{-1}\right)\left(q^{n} \tilde{\sigma}_{6}-q^{-n} l z^{-1}\right)}{\left(l-l^{-1}\right)^{2}} \\
& +\mathfrak{z}_{-} \frac{\left(z-z^{-1}\right)(z-l)\left(q^{n} \tilde{\sigma}_{6}-q^{-n} l^{-1} z^{-1}\right)}{\left(l-l^{-1}\right)^{2}} \\
& +[n]\left(z-z^{-1}\right)(z-l)\left(1-l^{-1} z^{-1}\right)\left(q^{n} \tilde{\sigma}_{6} z-q^{-n} z^{-1}\right) \\
& +\left[\tilde{\sigma}_{6} \tilde{\sigma}_{1}+\tilde{\sigma}_{5}-2 \tilde{\sigma}_{6}\left(l+l^{-1}\right)\right]\left(z-z^{-1}\right)(z-l)\left(1-l^{-1} z^{-1}\right) \\
& +\frac{1}{2} w_{+} \frac{(z+1)(z-l)\left(z^{-1}-l\right)\left(q^{n} \tilde{\sigma}_{6}-q^{-n} z^{-1}\right)}{(l-1)^{2}} \\
& -\frac{1}{2} w_{-} \frac{(z-1)(z-l)\left(z^{-1}-l\right)\left(q^{n} \tilde{\sigma}_{6}+q^{-n} z^{-1}\right)}{(l+1)^{2}}
\end{aligned}
$$


and

$$
\begin{aligned}
{[n] \mathfrak{W}_{-} } & (z ; t) \\
= & \mathfrak{z}_{-} \frac{\left(z-z^{-1}\right)\left(z-l^{-1}\right)\left(q^{n} \tilde{\sigma}_{6} l z^{-1}-q^{-n}\right)}{\left(l-l^{-1}\right)^{2}} \\
& +\mathfrak{z}_{+} \frac{\left(z-z^{-1}\right)(z-l)\left(q^{n} \tilde{\sigma}_{6} l^{-1} z^{-1}-q^{-n}\right)}{\left(l-l^{-1}\right)^{2}} \\
& -[n]\left(z-z^{-1}\right)(z-l)\left(1-l^{-1} z^{-1}\right)\left(q^{n} \tilde{\sigma}_{6} z^{-1}-q^{-n} z\right) \\
& -\left[\tilde{\sigma}_{6} \tilde{\sigma}_{1}+\tilde{\sigma}_{5}-2 \tilde{\sigma}_{6}\left(l+l^{-1}\right)\right]\left(z-z^{-1}\right)(z-l)\left(1-l^{-1} z^{-1}\right) \\
& +\frac{1}{2} w_{+} \frac{(z+1)(z-l)\left(z^{-1}-l\right)\left(q^{n} \tilde{\sigma}_{6} z^{-1}-q^{-n}\right)}{(l-1)^{2}} \\
& +\frac{1}{2} w_{-} \frac{(z-1)(z-l)\left(z^{-1}-l\right)\left(q^{n} \tilde{\sigma}_{6} z^{-1}+q^{-n}\right)}{(l+1)^{2}} .
\end{aligned}
$$

Proof. These formulas can be viewed as Laurent interpolating polynomials satisfying the following evaluations at the given nodes:

$$
\begin{aligned}
& \mathfrak{T}_{ \pm}\left(z^{-1} ; t\right)=-\mathfrak{T}_{ \pm}(z ; t), \\
& \mathfrak{T}_{+}\left(l^{ \pm 1} ; t\right)=0, \\
& \mathfrak{T}_{+}( \pm 1 ; t)=0, \\
& \mathfrak{T}_{+}(z ; t) \underset{z \rightarrow \infty}{\sim} 2 a_{n}\left[n+\frac{1}{2}\right] z^{2}, \\
& \mathfrak{T}_{+}(z ; t) \underset{z \rightarrow 0}{\sim}-2 a_{n}\left[n+\frac{1}{2}\right] z^{-2},
\end{aligned}
$$

and

$$
\begin{aligned}
& \mathfrak{W}_{ \pm}\left(z^{-1} ; t\right)=\mathfrak{W}_{\mp}(z ; t), \\
& \mathfrak{W}_{ \pm}(l ; t)=\mathfrak{z}_{ \pm}, \\
& \mathfrak{W}_{ \pm}( \pm 1 ; t)=w_{ \pm}, \\
& \mathfrak{W}_{+}(z ; t) \sim \underset{z \rightarrow \infty}{\sim} q^{n} \tilde{\sigma}_{6} z^{3}, \\
& \mathfrak{W}_{+}(z ; t) \underset{z \rightarrow 0}{\sim} q^{-n} z^{-3} .
\end{aligned}
$$

The above formulas are also a consequence of the parameterization (7.28)(7.29). 
REMARK 7.3. We note that the eigenvalues of $A_{n}^{*}(z ; t)$ as $z \rightarrow 0, \infty$ are $q^{n} \tilde{\sigma}_{6}, q^{-n}$, while those at the fixed points of the lattice, of the matrix $A_{n}^{*}( \pm 1 ; t)$, are $w_{ \pm}$. Under the mapping interchanging the interior and exterior of the unit circle in the spectral variable $z \mapsto 1 / z$, we observe that $\mathfrak{T}_{ \pm} \mapsto-\mathfrak{T}_{ \pm}$and $\mathfrak{W}_{ \pm} \mapsto \mathfrak{W}_{\mp}$. As a consequence $A_{n}^{*}(z ;) A_{n}^{*}\left(z^{-1} ;\right)=\left(W^{2}-\right.$ $\left.\Delta y^{2} V^{2}\right) \mathrm{Id}$, and therefore the mapping corresponds to a reversal of direction on the spectral lattice. With respect to the mapping $l \mapsto l^{-1}$ we note that this must be taken with $\mathfrak{z}_{+} \leftrightarrow \mathfrak{z}_{-}$, and conclude that $\mathfrak{T}_{+}, \mathfrak{W}_{ \pm}$are invariant under this type of transformation. In addition, $A_{n}^{*}$ is symmetrical under $t \mapsto t^{-1}$.

\subsection{Deformation structure}

The deformation data polynomials $R(x ; u), S(x ; u)$ are computed as

$$
R \pm \Delta v S=\left(1-q^{-1 / 2} \alpha t^{ \pm 1} z\right)\left(1-q^{-1 / 2} \alpha t^{ \pm 1} z^{-1}\right)=\phi_{1}\left(x ; q^{-1 / 2} \alpha t^{ \pm 1}\right)
$$

which implies

$$
\begin{aligned}
& R=1-\alpha^{2} q^{-1}-2 \alpha q^{-1 / 2} x u+2 \alpha^{2} q^{-1} u^{2}, \\
& S=2 \frac{\alpha}{q-1}\left(\alpha q^{-1 / 2} u-x\right),
\end{aligned}
$$

indicating that $L=1$. We observe that the polynomial

$$
R^{2}-\Delta v^{2} S^{2}=4 q^{-1} \alpha^{2}\left(x-\tilde{x}_{5}\right)\left(x-\tilde{x}_{6}\right)
$$

divides

$$
W^{2}-\Delta y^{2} V^{2}=64 q^{-3} \sigma_{4} \alpha^{2} \prod_{j=1}^{4}\left(x-\tilde{x}_{j}\right) \cdot\left(x-\tilde{x}_{5}\right)\left(x-\tilde{x}_{6}\right),
$$

in conformity with Remark 6.3 . The role of the common zeros $\tilde{x}_{5}, \tilde{x}_{6}$ will be crucial in the ensuing investigations. We also note that these points can be represented on a $u$-lattice, thus $\tilde{x}_{5}=E_{u}^{-} x_{5}$ and $\tilde{x}_{6}=E_{u}^{+} x_{6}$.

We can compute the remaining deformation data polynomial $T(x ; u)$, which will be of degree zero.

Proposition 7.7 ([97, Section 3.2]). The deformation data polynomial $T(x ; u)$ has the evaluation

$$
T(x ; u)=\frac{4 \alpha}{q-1} \frac{t m_{0,0}\left(v_{-}\right)-t^{-1} m_{0,0}\left(v_{+}\right)}{t-t^{-1}} .
$$


Proof. We start from the formula

$$
\Delta v T(x ; u)=(R-\Delta v S) f\left(x ; v_{+}\right)-(R+\Delta v S) f\left(x ; v_{-}\right)
$$

and employ the expansion (3.46) for some parameter $a$. Setting $a$ to one of $a_{1}, a_{2}, a_{3}, a_{4}$ and utilizing the identity

$$
\frac{\phi_{1}(x ; b)}{\phi_{n+1}(x ; a)}=\frac{\left(1-a b q^{n}\right)\left(1-b a^{-1} q^{-n}\right)}{\phi_{n+1}(x ; a)}+\frac{b a^{-1} q^{-n}}{\phi_{n}(x ; a)},
$$

we find, by equating coefficients, the result (7.75). Note that $m_{0,0}$ does not depend on $a$. If one chooses $a$ from $a_{5}, a_{6}$, then one requires the additional identity

$$
\frac{1}{\phi_{n}(x ; q a)}=\frac{\left(1-q^{n}\right)\left(a^{2}-q^{-n}\right)}{\phi_{n+1}(x ; a)}+\frac{q^{-n}}{\phi_{n}(x ; a)}
$$

in order to merge the two series in (7.76). Alternatively, one can compute $T$ from the initial value (6.43) using the asymptotic expression for $\Phi_{0}$ as given by $(6.33)$.

As a check, we can verify that the spectral data polynomials $W, V, U$ and the deformation data polynomials $R, S, T$ satisfy all of the consistency relations formulated in Section 6.

Corollary 7.1. The spectral data polynomials $W, V, U$ and the deformation data polynomials $R, S, T$ satisfy the consistency relations (6.4) and (6.16).

Proof. The first task involving $W, V$ and $R, S$ is elementary, whereas the second requires a moment relation

$$
\begin{aligned}
& {[1]\left(m_{0,+}+m_{0,-}\right)(t)} \\
& =\left[q^{-2} \alpha^{2} \sigma_{3}-q^{-1} \sigma_{1}+q^{-3}\left(q-\alpha^{2}\right)\left(q^{2} \alpha^{-1} t^{-1}+\sigma_{4} \alpha t\right)\right] m_{0,0}(t) \\
& \quad-\frac{w\left(q^{-1 / 2} \alpha t\right)}{\alpha t} \frac{q^{-1 / 2} t m_{0,0}\left(q^{-1} t\right)-q^{1 / 2} t^{-1} m_{0,0}(t)}{q^{-1 / 2} t-q^{1 / 2} t^{-1}}
\end{aligned}
$$

and the moment $q$-difference equation in $t$

$$
\begin{aligned}
& \left(q^{-1} t-q t^{-1}\right) w\left(\alpha t^{-1}\right) m_{0,0}\left(q^{1 / 2} t\right) \\
& \quad-q^{-1 / 2}\left(q^{-1 / 2} t-q^{1 / 2} t^{-1}\right)\left[(1+q)\left(1+q^{-1} \alpha^{2} \sigma_{2}+q^{-2} \alpha^{4} \sigma_{4}\right)\right.
\end{aligned}
$$




$$
\begin{aligned}
& +q^{-1} \alpha^{2}\left(q+\sigma_{4}\right)\left(t-t^{-1}\right)\left(q^{-1} t-q t^{-1}\right) \\
& \left.-\alpha\left(q^{-1 / 2} t+q^{1 / 2} t^{-1}\right)\left(\sigma_{1}+q^{-1} \alpha^{2} \sigma_{3}\right)\right] m_{0,0}\left(q^{-1 / 2} t\right) \\
& +\left(t-t^{-1}\right) w\left(q^{-1} \alpha t\right) m_{0,0}\left(q^{-3 / 2} t\right)=0,
\end{aligned}
$$

which is just (7.8) with $a_{5} \mapsto q^{-3 / 2} \alpha t, a_{6} \mapsto q^{-1 / 2} \alpha t^{-1}$.

We know for $L=1$ that the deformation coefficients can be parameterized, thus

$$
\begin{aligned}
& \mathfrak{R}_{ \pm}=r_{1 \pm} x+r_{0 \pm} \\
& \mathfrak{P}_{ \pm}=p_{ \pm}
\end{aligned}
$$

Furthermore, we know from the large $x$ expansions (6.32)-(6.36) what the leading terms are, however in contrast to those of the spectral coefficients, these are related to the three-term recurrence coefficients $a_{n}, \gamma_{n}$ in a nontrivial way.

Corollary 7.2. The leading order terms of the elements of the matrix $B_{n}^{*}$ are given by

$$
\begin{aligned}
& r_{1+}=4 \alpha q^{-1 / 2} H_{n} \frac{t \gamma_{n}\left(v_{+}\right)}{\gamma_{n-1}\left(v_{-}\right)}, \\
& r_{1-}=4 \alpha q^{-1 / 2} H_{n} \frac{\gamma_{n}\left(v_{-}\right)}{t \gamma_{n-1}\left(v_{+}\right)}, \\
& p_{+}=4 \alpha q^{-1 / 2} H_{n}\left[\frac{t \gamma_{n}\left(v_{+}\right)}{\gamma_{n}\left(v_{-}\right)}-\frac{\gamma_{n}\left(v_{-}\right)}{t \gamma_{n}\left(v_{+}\right)}\right] \\
& p_{-}=4 \alpha q^{-1 / 2} \frac{a_{n} H_{n-1}}{a_{n-1}}\left[\frac{t \gamma_{n-1}\left(v_{+}\right)}{\gamma_{n-1}\left(v_{-}\right)}-\frac{\gamma_{n-1}\left(v_{-}\right)}{t \gamma_{n-1}\left(v_{+}\right)}\right]
\end{aligned}
$$

for $n \geq 1$, while for $n=0$ one can use $\gamma_{-1}=a_{0} \gamma_{0}$.

Up to this point we have not exercised a choice regarding the decoupling factor $H_{n}$, but one can take $H_{n}=\frac{1}{2} a_{n}$ henceforth.

REMARK 7.4. We note that the eigenvalues of $B_{n}^{*}(z ; t)$ as $z \rightarrow 0, \infty$ are $r_{1 \pm}$. By construction, $B_{n}^{*}$ is symmetrical with respect to $z \mapsto z^{-1}$. Under the mapping interchanging the interior and exterior of the unit circle in the 
deformation variable $t \mapsto 1 / t$, we observe (see (6.54), (6.55), and (6.56)) that $\mathfrak{P}_{ \pm} \mapsto-\mathfrak{P}_{ \pm}$and $\mathfrak{R}_{ \pm} \mapsto \mathfrak{R}_{\mp}$. As a consequence

$$
B_{n}^{*}(; t) B_{n}^{*}\left(; t^{-1}\right)=\frac{4 H_{n}^{2}}{a_{n}\left(v_{-}\right) a_{n}\left(v_{+}\right)}\left(R^{2}-\Delta v^{2} S^{2}\right) \mathrm{Id}
$$

and therefore this mapping corresponds to a reversal of direction on the deformation lattice.

As part of an efficient methodology, we will require formulas which relate the lower order terms, with respect to $x$, of the deformation coefficients to the leading order term.

Proposition 7.8. The trailing terms of the deformation coefficients are related to each other and to the spectral parameterization by

$$
\begin{aligned}
p_{+}= & r_{1+} a_{n}\left(v_{-}\right)-r_{1-} a_{n}\left(v_{+}\right), \\
p_{-}= & r_{1+} a_{n}\left(v_{+}\right)-r_{1-} a_{n}\left(v_{-}\right), \\
r_{0+}= & \frac{r_{1+}}{\Delta v_{2}}\left[-w_{2}\left(v_{+}\right)+w_{2}\left(v_{-}\right)\right. \\
& \left.-2 \sqrt{\Delta}\left(q^{-1 / 2} \alpha+q^{1 / 2} \alpha^{-1}\right)\left(\tilde{\sigma}_{6} q^{n} t-q^{-n} t^{-1}\right)\right], \\
r_{0-}= & \frac{r_{1-}}{\Delta v_{2}}\left[w_{2}\left(v_{+}\right)-w_{2}\left(v_{-}\right)\right. \\
& \left.-2 \sqrt{\Delta}\left(q^{-1 / 2} \alpha+q^{1 / 2} \alpha^{-1}\right)\left(\tilde{\sigma}_{6} q^{n} t^{-1}-q^{-n} t\right)\right] .
\end{aligned}
$$

Proof. The formulas given above are ones which involve both shifts up and down on the $u$-lattice of the spectral variables, and these are easily derived by resolving the compatibility relation (6.67) as a Laurent polynomial in $z$. Such a polynomial is constructed in our application by substituting the elements (7.57)-(7.59) and (7.79) and (7.80) into (6.67) and collecting terms. For the $(1,2)$ component, we find that the leading order nonzero contributions occur at $z^{ \pm 7}$ and the coefficients of both these terms will vanish if $(7.86)$ holds. In the case of the $(2,1)$ component, the $z^{ \pm 7}$ terms are the leading nontrivial ones and these both vanish when (7.87) holds. The two latter relations (7.88) and (7.89) follow from the requirement that the coefficients of the $z^{7}$ terms of the $(1,1)$ and $(2,2)$ elements vanish, respectively. Finally, we observe that an independent way of verifying (7.86) and (7.87) is through a trivial combination of the formulas (7.81)-(7.84). 
A vital part of our analysis consists of resolving the compatibility relations (6.70)-(6.73) with respect to the zeros and poles of $\chi(z ; t)$.

COROLlary 7.3. The residues of the compatibility relation given in Proposition 6.11 consist of the following equations:

(i) at the advanced coordinate $z=z_{5}=\alpha t$

$$
\begin{aligned}
& r_{1-} E_{u}^{-} x_{5}+r_{0-}=-\frac{\mathfrak{W}_{+}\left(z_{5} ; q^{1 / 2} t\right)}{\mathfrak{T}_{+}\left(z_{5} ; q^{1 / 2} t\right)} p_{+}, \\
& r_{1+} E_{u}^{-} x_{5}+r_{0+}=-\frac{\mathfrak{W}_{-}\left(z_{5} ; q^{1 / 2} t\right)}{\mathfrak{T}_{-}\left(z_{5} ; q^{1 / 2} t\right)} p_{-},
\end{aligned}
$$

(ii) at the advanced coordinate $z=E_{u}^{2+} z_{6}=q^{-1} \alpha t^{-1}$

$$
\begin{aligned}
& r_{1-} E_{u}^{+} x_{6}+r_{0-}=\frac{\mathfrak{W}_{-}\left(E_{u}^{2+} z_{6} ; q^{1 / 2} t\right)}{\mathfrak{T}_{+}\left(E_{u}^{2+} z_{6} ; q^{1 / 2} t\right)} p_{+}, \\
& r_{1+} E_{u}^{+} x_{6}+r_{0+}=\frac{\mathfrak{W}_{+}\left(E_{u}^{2+} z_{6} ; q^{1 / 2} t\right)}{\mathfrak{T}_{-}\left(E_{u}^{2+} z_{6} ; q^{1 / 2} t\right)} p_{-},
\end{aligned}
$$

(iii) at the retarded coordinate $z=E_{u}^{2-} z_{5}=q^{-1} \alpha t$

$$
\begin{aligned}
& r_{1+} E_{u}^{-} x_{5}+r_{0+}=-\frac{\mathfrak{W}_{-}\left(E_{u}^{2-} z_{5} ; q^{-1 / 2} t\right)}{\mathfrak{T}_{+}\left(E_{u}^{2-} z_{5} ; q^{-1 / 2} t\right)} p_{+}, \\
& r_{1-} E_{u}^{-} x_{5}+r_{0-}=-\frac{\mathfrak{W}_{+}\left(E_{u}^{2-} z_{5} ; q^{-1 / 2} t\right)}{\mathfrak{T}_{-}\left(E_{u}^{2-} z_{5} ; q^{-1 / 2} t\right)} p_{-},
\end{aligned}
$$

(iv) and at the retarded coordinate $z=z_{6}=\alpha t^{-1}$

$$
\begin{aligned}
& r_{1+} E_{u}^{+} x_{6}+r_{0+}=\frac{\mathfrak{W}_{+}\left(z_{6} ; q^{-1 / 2} t\right)}{\mathfrak{T}_{+}\left(z_{6} ; q^{-1 / 2} t\right)} p_{+}, \\
& r_{1-} E_{u}^{+} x_{6}+r_{0-}=\frac{\mathfrak{W}_{-}\left(z_{6} ; q^{-1 / 2} t\right)}{\mathfrak{T}_{-}\left(z_{6} ; q^{-1 / 2} t\right)} p_{-} .
\end{aligned}
$$

All the deformation parameters are evaluated at $t, u$, that is, $p_{ \pm}(t), r_{1 \pm}(t)$, $r_{0 \pm}(t)$.

Proof. The multiplicative factor (6.17) is given, in this case, by

$$
\chi(z ; t)=\frac{\left(1-q^{-1} \alpha t^{-1} z\right)\left(1-\alpha t z^{-1}\right)}{\left(1-q^{-1} \alpha t z^{-1}\right)\left(1-\alpha t^{-1} z\right)} .
$$


At the zeros of $\chi$ the right-hand sides of (6.70), (6.71), (6.72), and (6.73) must vanish, whereas at the poles the left-hand factors must vanish. Thus for the zero $z=z_{5}=\alpha t$ applied to (6.70), we deduce (7.90). For the other zero at $z=1 / E_{u}^{2+} z_{6}=q \alpha^{-1} t$ applied to (6.70), we make additional use of the symmetries of $A_{n}^{*}$ and $B_{n}^{*}$ with respect to inversion of $z$ to derive (7.92). At the pole $z=E_{u}^{2-} z_{5}=q^{-1} \alpha t$ the residue of (6.70) yields (7.94), whereas at the other pole $z=1 / z_{6}=\alpha^{-1} t$ we have to apply the additional symmetries to the residue to arrive at (7.96). However we have further relations, which are equivalent to the four relations derived above, by specializing the spectral variable in these ways due to the fact that $\left(W^{2}-\Delta y^{2} V^{2}\right)(z=$ $\left.\alpha t, q^{-1} \alpha t^{-1} ; q^{1 / 2} t\right)=0,\left(W^{2}-\Delta y^{2} V^{2}\right)\left(z=q^{-1} \alpha t, \alpha t^{-1} ; q^{-1 / 2} t\right)=0,\left(R^{2}-\right.$ $\left.\Delta v^{2} S^{2}\right)\left(z=q^{-1 / 2} \alpha t ; t\right)=0$, and $\left(R^{2}-\Delta v^{2} S^{2}\right)\left(z=q^{-1 / 2} \alpha t^{-1} ; t\right)=0$, which implies

$$
\begin{aligned}
\left(\mathfrak{W}_{+} \mathfrak{W}_{-}+\mathfrak{T}_{+} \mathfrak{T}_{-}\right)\left(z=\alpha t, q^{-1} \alpha t^{-1} ; q^{1 / 2} t\right) & =0, \\
\left(\mathfrak{W}_{+} \mathfrak{W}_{-}+\mathfrak{T}_{+} \mathfrak{T}_{-}\right)\left(z=q^{-1} \alpha t, \alpha t^{-1} ; q^{-1 / 2} t\right) & =0, \\
\left(\mathfrak{R}_{+} \mathfrak{R}_{-}+\mathfrak{P}_{+} \mathfrak{P}_{-}\right)\left(z=q^{-1 / 2} \alpha t ; t\right) & =0, \\
\left(\mathfrak{R}_{+} \mathfrak{R}_{-}+\mathfrak{P}_{+} \mathfrak{P}_{-}\right)\left(z=q^{-1 / 2} \alpha t^{-1} ; t\right) & =0 .
\end{aligned}
$$

This means that each of the identities (7.90), (7.92), (7.94), and (7.96) can take four forms and that the remaining relations (7.91), (7.93), (7.95), and (7.97) are examples of just one of those forms. This equivalence also ensures that if the residue condition is satisfied by (6.70), then it is automatically satisfied by (6.71), (6.72), and (6.73) as well.

COROLlary 7.4. The following product relation holds among the spectral coefficients evaluated at the zeros of the spectral determinant

$$
\begin{aligned}
& \frac{\mathfrak{W}_{+}\left(z_{5} ; q^{1 / 2} t\right)}{\mathfrak{T}_{+}\left(z_{5} ; q^{1 / 2} t\right)} \frac{\mathfrak{W}_{-}\left(E_{u}^{2-} z_{5} ; q^{-1 / 2} t\right)}{\mathfrak{T}_{+}\left(E_{u}^{2-} z_{5} ; q^{-1 / 2} t\right)} \\
& \quad=\frac{\mathfrak{W}_{+}\left(z_{6} ; q^{-1 / 2} t\right)}{\mathfrak{T}_{+}\left(z_{6} ; q^{-1 / 2} t\right)} \frac{\mathfrak{W}_{-}\left(E_{u}^{2+} z_{6} ; q^{1 / 2} t\right)}{\mathfrak{T}_{+}\left(E_{u}^{2+} z_{6} ; q^{1 / 2} t\right)} .
\end{aligned}
$$

Proof. By comparing the right-hand sides of the pairs (7.90) and (7.95), (7.92) and (7.97), (7.94) and (7.91), and (7.96) and (7.93), we see that

$$
\frac{\mathfrak{W}_{+}\left(z_{5} ; q^{1 / 2} t\right)}{\mathfrak{T}_{+}\left(z_{5} ; q^{1 / 2} t\right)} p_{+}=\frac{\mathfrak{W}_{+}\left(E_{u}^{2-} z_{5} ; q^{-1 / 2} t\right)}{\mathfrak{T}_{-}\left(E_{u}^{2-} z_{5} ; q^{-1 / 2} t\right)} p_{-},
$$




$$
\begin{aligned}
\frac{\mathfrak{W}_{-}\left(E_{u}^{2+} z_{6} ; q^{1 / 2} t\right)}{\mathfrak{T}_{+}\left(E_{u}^{2+} z_{6} ; q^{1 / 2} t\right)} p_{+} & =\frac{\mathfrak{W}_{-}\left(z_{6} ; q^{-1 / 2} t\right)}{\mathfrak{T}_{-}\left(z_{6} ; q^{-1 / 2} t\right)} p_{-}, \\
\frac{\mathfrak{W}_{-}\left(E_{u}^{2-} z_{5} ; q^{-1 / 2} t\right)}{\mathfrak{T}_{+}\left(E_{u}^{2-} z_{5} ; q^{-1 / 2} t\right)} p_{+} & =\frac{\mathfrak{W}_{-}\left(z_{5} ; q^{1 / 2} t\right)}{\mathfrak{T}_{-}\left(z_{5} ; q^{1 / 2} t\right)} p_{-}, \\
\frac{\mathfrak{W}_{+}\left(z_{6} ; q^{-1 / 2} t\right)}{\mathfrak{T}_{+}\left(z_{6} ; q^{-1 / 2} t\right)} p_{+} & =\frac{\mathfrak{W}_{+}\left(E_{u}^{2+} z_{6} ; q^{1 / 2} t\right)}{\mathfrak{T}_{-}\left(E_{u}^{2+} z_{6} ; q^{1 / 2} t\right)} p_{-\cdot} .
\end{aligned}
$$

By forming the cross products of the first and second relations, or equivalently the third and fourth, we can establish (7.103), assuming $p_{+} p_{-} \neq 0$. Taking cross products of the first and third, or second and fourth leads to the identities that are trivial consequences of the determinantal relation.

DEFINITION 7.2. In conformity with the remark made immediately preceding Corollary 6.4, let us define the variables with deformation arguments evaluated at the advanced and retarded coordinates through the notation $\hat{l}=l\left(q^{1 / 2} t\right), \check{l}=l\left(q^{-1 / 2} t\right)$, and so on.

LEMMA 7.1. One solution for the component $p_{+}$is given by

$$
\begin{aligned}
q^{1 / 2} & \alpha^{-1}\left(t^{-1}-t\right)\left[n+\frac{1}{2}\right] \frac{r_{1-\hat{a}_{n}}}{p_{+}} \\
= & -\frac{q t \hat{l}}{\left(\hat{l}-\hat{l}^{-1}\right)^{2}}\left[\frac{\hat{\mathfrak{z}}_{+}}{(\alpha t-\hat{l})(q t-\alpha \hat{l})}+\frac{\hat{\mathfrak{z}}_{-}}{(-\alpha+q t \hat{l})(-1+\alpha t \hat{l})}\right] \\
& \quad-\frac{1}{\alpha t}\left(q^{-n}+q^{n+1} t^{2} \sigma_{6}\right)+\frac{1}{4} w(1) \frac{1}{\hat{\lambda}-1}+\frac{1}{4} w(-1) \frac{1}{\hat{\lambda}+1} .
\end{aligned}
$$

A second solution for the component $p_{+}$is

$$
\begin{aligned}
& q^{1 / 2} \alpha^{-1}\left(t^{-1}-t\right)\left[n+\frac{1}{2}\right] \frac{r_{1+} \check{a}_{n}}{p_{+}} \\
&=\frac{q t \check{l}}{\left(\check{l}-\check{l}^{-1}\right)^{2}}\left[\frac{\check{\check{\mathfrak{z}}}+}{(q-\alpha t \check{l})(-\alpha+t \check{l})}+\frac{\check{\mathfrak{z}}-}{(t-\alpha \check{l})(-\alpha t+q \check{l})}\right] \\
&-\frac{1}{\alpha t}\left(q^{-n} t^{2}+q^{n+1} \sigma_{6}\right)+\frac{1}{4} w(1) \frac{1}{\check{\lambda}-1}+\frac{1}{4} w(-1) \frac{1}{\check{\lambda}+1} .
\end{aligned}
$$

Proof. The first relation (7.108) follows from the subtraction of (7.92) from (7.90) and simplifying. The second relation (7.109) follows from similar reasoning applied to (7.94) and (7.96). 
REMARK 7.5. A profound difference between the expressions (7.108) and (7.109) and the corresponding formulas in the case of the $q$-linear spectral lattice is the presence of both $\hat{\mathfrak{z}}_{ \pm}\left(\right.$or $\left.\breve{\mathfrak{z}}_{ \pm}\right)$, whereas in the latter case only one of these variables is present, which significantly complicates the ensuing analysis.

Definition 7.3. We define the following sequence of coordinate transformations

$$
\begin{aligned}
& \mathfrak{z}_{+}=l^{-2} w(l)\left(1-q^{-1 / 2} \alpha t l^{-1}\right)\left(1-q^{-1 / 2} \alpha t^{-1} l\right) \frac{l f-1}{f-l}, \\
& \mathfrak{z}_{-}=l^{2} w\left(l^{-1}\right)\left(1-q^{-1 / 2} \alpha t l\right)\left(1-q^{-1 / 2} \alpha t^{-1} l^{-1}\right) \frac{f-l}{l f-1},
\end{aligned}
$$

where the new variable $f(t)$ is introduced. Clearly, it is apparent that under the mapping $l \mapsto l^{-1}$ we have the interchange $\mathfrak{z}_{+} \leftrightarrow \mathfrak{z}_{-}$and that the product satisfies the relation $(7.27)$, that is, $\mathfrak{z}_{+} \mathfrak{z}_{-}=\prod_{j=1}^{6}\left(1-q^{-1 / 2} a_{j} l^{ \pm}\right)$. Let $\sigma_{4}=$ $q^{2} s_{4}^{2}$. In addition, we define the further variable $\rho(t)$ so that

$$
\begin{aligned}
& \frac{r_{1+} \check{a}_{n}}{p_{+}}:=\frac{1}{q^{1 / 2}\left[n+\frac{1}{2}\right]} \frac{2 \alpha s_{4} \hat{\rho}-q^{-n} t-q^{n} \alpha^{2} s_{4}^{2} t^{-1}}{t-t^{-1}}, \\
& \frac{r_{1-\hat{a}_{n}}}{p_{+}}:=\frac{1}{q^{1 / 2}\left[n+\frac{1}{2}\right]} \frac{2 \alpha s_{4} \hat{\rho}-q^{n} \alpha^{2} s_{4}^{2} t-q^{-n} t^{-1}}{t-t^{-1}} .
\end{aligned}
$$

Together these relations ensure that (7.86) is automatically satisfied. We further define $g(t)$ by $2 \rho:=g+g^{-1}$. We will find that $f, l, g$ will be our primary variables.

A consequence of these definitions and Lemma 7.1 is the following transformation formula.

Corollary 7.5. Let us assume that $f \neq 0, \infty, f \neq l^{ \pm 1}$, and $w(f) \neq 0$. There exists an invertible mapping between $\rho(t)$ and $\lambda(t)$ with the form

$$
-2 s_{4} f \rho+1+s_{4}^{2} f^{2}=\frac{w(f)}{f^{2}+1-2 f \lambda} .
$$

Proof. Employing the definitions (7.113), (7.110), and (7.111) in (7.108), we compute the relation

$$
2 s_{4} \rho=q^{-3 / 2} \sigma_{3}-s_{4}^{2}\left[l+l^{-1}\right]+\frac{w(l)}{\left(l^{2}-1\right)(l-f)}+\frac{l^{3} w\left(l^{-1}\right)}{\left(l^{2}-1\right)(l f-1)}
$$


where we have shifted the arguments $t \mapsto q^{-1 / 2} t$ of all variables. The relation (7.114) then follows by performing a partial fraction expansion of the above equation with respect to the variable $l(t)$ (the former equation can be thought of as an expansion with respect to $f(t)$ ). We also have an alternative form of the above expression

$$
\begin{aligned}
\lambda= & q^{1 / 2} \frac{\sigma_{3}}{2 \sigma_{4}}-\frac{1}{2 s_{4}}\left(g+g^{-1}\right) \\
& -\frac{1}{2\left(g^{2}-1\right)} \frac{w\left(s_{4}^{-1} g\right)}{f-s_{4}^{-1} g}+\frac{g^{2}}{2\left(g^{2}-1\right)} \frac{w\left(s_{4}^{-1} g^{-1}\right)}{f-s_{4}^{-1} g^{-1}} .
\end{aligned}
$$

REMARK 7.6. What is surprising about this result in comparison to the situation with the $q$-linear lattice is that instead of a linear transformation between $\rho$ and $f$, we find one between $\rho$ and $\lambda$ with $f$ being an intermediary. Given (7.115), it is clear that there is no linear inversion possible for $f$.

Our next task is to compute expressions for the four independent evaluations of the spectral matrix elements given on the left-hand sides of (7.104)(7.107) in terms of the variables introduced above. We will carry this out in stages of successive refinement.

LEMMA 7.2. The ratios of the evaluated spectral matrix elements satisfy the relations

$$
\begin{aligned}
& \frac{\hat{a}_{n}}{\check{a}_{n}} \frac{2 \alpha s_{4} \hat{\rho}-q^{-n} t-q^{n} \alpha^{2} s_{4}^{2} t^{-1}}{2 \alpha s_{4} \hat{\rho}-q^{n} \alpha^{2} s_{4}^{2} t-q^{-n} t^{-1}} \\
& \quad=\frac{\mathfrak{W}_{+}\left(z_{6} ; q^{-1 / 2} t\right)}{\mathfrak{T}_{+}\left(z_{6} ; q^{-1 / 2} t\right)} \frac{\mathfrak{T}_{+}\left(z_{5} ; q^{1 / 2} t\right)}{\mathfrak{W}_{+}\left(z_{5} ; q^{1 / 2} t\right)} \\
& \quad=\frac{\mathfrak{W}_{-}\left(E_{u}^{2-} z_{5} ; q^{-1 / 2} t\right)}{\mathfrak{T}_{+}\left(E_{u}^{2-} z_{5} ; q^{-1 / 2} t\right)} \frac{\mathfrak{T}_{+}\left(E_{u}^{2+} z_{6} ; q^{1 / 2} t\right)}{\mathfrak{W}_{-}\left(E_{u}^{2+} z_{6} ; q^{1 / 2} t\right)} .
\end{aligned}
$$

Proof. Subtracting (7.90) from (7.92) gives

$$
r_{1-}\left(E_{u}^{+} x_{6}-E_{u}^{-} x_{5}\right)=\left(\frac{\mathfrak{W}_{-}\left(E_{u}^{2+} z_{6} ; q^{1 / 2} t\right)}{\mathfrak{T}_{+}\left(E_{u}^{2+} z_{6} ; q^{1 / 2} t\right)}+\frac{\mathfrak{W}_{+}\left(z_{5} ; q^{1 / 2} t\right)}{\mathfrak{T}_{+}\left(z_{5} ; q^{1 / 2} t\right)}\right) p_{+},
$$

while subtracting (7.94) from (7.96) gives

$$
r_{1+}\left(E_{u}^{+} x_{6}-E_{u}^{-} x_{5}\right)=\left(\frac{\mathfrak{W}_{+}\left(z_{6} ; q^{-1 / 2} t\right)}{\mathfrak{T}_{+}\left(z_{6} ; q^{-1 / 2} t\right)}+\frac{\mathfrak{W}_{-}\left(E_{u}^{2-} z_{5} ; q^{-1 / 2} t\right)}{\mathfrak{T}_{+}\left(E_{u}^{2-} z_{5} ; q^{-1 / 2} t\right)}\right) p_{+}
$$


Taking ratios of these two gives

$$
\frac{r_{1+}}{r_{1-}}=\frac{\mathfrak{T}_{+}\left(z_{5} ; q^{1 / 2} t\right) \mathfrak{T}_{+}\left(E_{u}^{2+} z_{6} ; q^{1 / 2} t\right)}{\mathfrak{T}_{+}\left(z_{6} ; q^{-1 / 2} t\right) \mathfrak{T}_{+}\left(E_{u}^{2-} z_{5} ; q^{-1 / 2} t\right)}
$$

$$
\times \frac{\mathfrak{T}_{+}\left(z_{6} ; q^{-1 / 2} t\right) \mathfrak{W}_{-}\left(E_{u}^{2-} z_{5} ; q^{-1 / 2} t\right)+\mathfrak{T}_{+}\left(E_{u}^{2-} z_{5} ; q^{-1 / 2} t\right) \mathfrak{W}_{+}\left(z_{6} ; q^{-1 / 2} t\right)}{\mathfrak{T}_{+}\left(z_{5} ; q^{1 / 2} t\right) \mathfrak{W}_{-}\left(E_{u}^{2+} z_{6} ; q^{1 / 2} t\right)+\mathfrak{T}_{+}\left(E_{u}^{2+} z_{6} ; q^{1 / 2} t\right) \mathfrak{W}_{+}\left(z_{5} ; q^{1 / 2} t\right)} .
$$

Likewise, subtracting (7.91) from (7.93) yields

$$
\begin{aligned}
r_{1+} & \left(E_{u}^{+} x_{6}-E_{u}^{-} x_{5}\right) \\
& =-\left(\frac{\mathfrak{T}_{+}\left(E_{u}^{2+} z_{6} ; q^{1 / 2} t\right)}{\mathfrak{W}_{-}\left(E_{u}^{2+} z_{6} ; q^{1 / 2} t\right)}+\frac{\mathfrak{T}_{+}\left(z_{5} ; q^{1 / 2} t\right)}{\mathfrak{W}_{+}\left(z_{5} ; q^{1 / 2} t\right)}\right) p_{-},
\end{aligned}
$$

and (7.95) from (7.97)

$$
\begin{aligned}
r_{1-} & \left(E_{u}^{+} x_{6}-E_{u}^{-} x_{5}\right) \\
& =-\left(\frac{\mathfrak{T}_{+}\left(z_{6} ; q^{-1 / 2} t\right)}{\mathfrak{W}_{+}\left(z_{6} ; q^{-1 / 2} t\right)}+\frac{\mathfrak{T}_{+}\left(E_{u}^{2-} z_{5} ; q^{-1 / 2} t\right)}{\mathfrak{W}_{-}\left(E_{u}^{2-} z_{5} ; q^{-1 / 2} t\right)}\right) p_{-} .
\end{aligned}
$$

Taking the ratio of these latter two equations gives

$$
\begin{aligned}
\frac{r_{1+}}{r_{1-}}= & \frac{\mathfrak{W}_{+}\left(z_{6} ; q^{-1 / 2} t\right) \mathfrak{W}_{-}\left(E_{u}^{2-} z_{5} ; q^{-1 / 2} t\right)}{\mathfrak{W}_{+}\left(z_{5} ; q^{1 / 2} t\right) \mathfrak{W}_{-}\left(E_{u}^{2+} z_{6} ; q^{1 / 2} t\right)} \\
(7.123) & \times \frac{\mathfrak{T}_{+}\left(z_{5} ; q^{1 / 2} t\right) \mathfrak{W}_{-}\left(E_{u}^{2+} z_{6} ; q^{1 / 2} t\right)+\mathfrak{T}_{+}\left(E_{u}^{2+} z_{6} ; q^{1 / 2} t\right) \mathfrak{W}_{+}\left(z_{5} ; q^{1 / 2} t\right)}{\mathfrak{T}_{+}\left(z_{6} ; q^{-1 / 2} t\right) \mathfrak{W}_{-}\left(E_{u}^{2-} z_{5} ; q^{-1 / 2} t\right)+\mathfrak{T}_{+}\left(E_{u}^{2-} z_{5} ; q^{-1 / 2} t\right) \mathfrak{W}_{+}\left(z_{6} ; q^{-1 / 2} t\right)}
\end{aligned}
$$

The factors appearing on the right-hand sides of these two ratios as constructed have simple evaluations, the first factor being

$$
\begin{aligned}
& \frac{\mathfrak{T}_{+}\left(z_{5} ; q^{1 / 2} t\right) \mathfrak{T}_{+}\left(E_{u}^{2+} z_{6} ; q^{1 / 2} t\right)}{\mathfrak{T}_{+}\left(z_{6} ; q^{-1 / 2} t\right) \mathfrak{T}_{+}\left(E_{u}^{2-} z_{5} ; q^{-1 / 2} t\right)} \\
& =\frac{\hat{a}_{n}^{2}}{\check{a}_{n}^{2}} \frac{\left(q^{2} t^{2}-\alpha^{2}\right)\left(\alpha^{2} t^{2}-1\right)(\alpha t-\hat{l})}{\left(t^{2}-\alpha^{2}\right)\left(\alpha^{2} t^{2}-q^{2}\right)(\alpha t-q \check{l})} \\
& \quad \times \frac{\left(\alpha t-\hat{l}^{-1}\right)\left(q t-\alpha \hat{l}^{-1}\right)(q t-\alpha \hat{l})}{\left(\alpha t-q \check{l}^{-1}\right)\left(t-\alpha \check{l}^{-1}\right)(t-\alpha \check{l})} .
\end{aligned}
$$


Employing this evaluation in the first ratio (7.120) yields the evaluation for the second factor

$$
\begin{aligned}
\frac{\mathfrak{T}_{+}\left(z_{6} ; q^{-1 / 2} t\right) \mathfrak{W}_{-}\left(E_{u}^{2-} z_{5} ; q^{-1 / 2} t\right)+\mathfrak{T}_{+}\left(E_{u}^{2-} z_{5} ; q^{-1 / 2} t\right) \mathfrak{W}_{+}\left(z_{6} ; q^{-1 / 2} t\right)}{\mathfrak{T}_{+}\left(z_{5} ; q^{1 / 2} t\right) \mathfrak{W}_{-}\left(E_{u}^{2+} z_{6} ; q^{1 / 2} t\right)+\mathfrak{T}_{+}\left(E_{u}^{2+} z_{6} ; q^{1 / 2} t\right) \mathfrak{W}_{+}\left(z_{5} ; q^{1 / 2} t\right)} \\
=\frac{\check{a}_{n}}{\hat{a}_{n}} \frac{\left(t^{2}-\alpha^{2}\right)\left(\alpha^{2} t^{2}-q^{2}\right)}{\left(q^{2} t^{2}-\alpha^{2}\right)\left(\alpha^{2} t^{2}-1\right)} \\
\quad \times \frac{(\alpha t-q \check{l})\left(\alpha t-q \check{l}^{-1}\right)\left(t-\alpha \check{l}^{-1}\right)(t-\alpha \check{l})}{(\alpha t-\hat{l})\left(\alpha t-\hat{l}^{-1}\right)\left(q t-\alpha \hat{l}^{-1}\right)(q t-\alpha \hat{l})} \\
\quad \times \frac{2 \alpha s_{4} \hat{\rho}-q^{-n} t-q^{n} \alpha^{2} s_{4}^{2} t^{-1}}{2 \alpha s_{4} \hat{\rho}-q^{n} \alpha^{2} s_{4}^{2} t-q^{-n} t^{-1}} .
\end{aligned}
$$

Employing this latter factor in the second ratio (7.123), we can construct two relations involving perfect squares, upon using (7.103). After taking the square roots, we need to resolve the sign ambiguity. The final result is then (7.117).

REMARK 7.7. In fact, we can make separate evaluations of the numerator and denominator of the last ratio, which gives us

$$
\begin{aligned}
\mathfrak{T}_{+} & \left(z_{5} ; q^{1 / 2} t\right) \mathfrak{W}_{-}\left(E_{u}^{2+} z_{6} ; q^{1 / 2} t\right)+\mathfrak{T}_{+}\left(E_{u}^{2+} z_{6} ; q^{1 / 2} t\right) \mathfrak{W}_{+}\left(z_{5} ; q^{1 / 2} t\right) \\
= & \frac{2 \hat{a}_{n}\left[n+\frac{1}{2}\right]}{q^{3} \alpha^{5} t^{4}}\left(q^{2} t^{2}-\alpha^{2}\right)\left(\alpha^{2} t^{2}-1\right)\left(\alpha^{2}-q\right) \\
& \times(\alpha t-\hat{l})\left(\alpha t-\hat{l}^{-1}\right)(q t-\alpha \hat{l})\left(q t-\alpha \hat{l}^{-1}\right) \\
& \times\left[2 \alpha s_{4} \hat{\rho}-q^{n} \alpha^{2} s_{4}^{2} t-q^{-n} t^{-1}\right]
\end{aligned}
$$

and

$$
\begin{aligned}
\mathfrak{T}_{+}\left(z_{6} ; q^{-1 / 2} t\right) \mathfrak{W}_{-}\left(E_{u}^{2-} z_{5} ; q^{-1 / 2} t\right) \\
\quad+\mathfrak{T}_{+}\left(E_{u}^{2-} z_{5} ; q^{-1 / 2} t\right) \mathfrak{W}_{+}\left(z_{6} ; q^{-1 / 2} t\right) \\
=\frac{2 \check{a}_{n}\left[n+\frac{1}{2}\right]}{q^{3} \alpha^{5} t^{4}}\left(t^{2}-\alpha^{2}\right)\left(\alpha^{2} t^{2}-q^{2}\right)\left(\alpha^{2}-q\right) \\
\quad \times(\alpha t-q \check{l})\left(\alpha t-q \check{l}^{-1}\right)(t-\alpha \check{l})\left(t-\alpha \check{l}^{-1}\right) \\
\quad \times\left[2 \alpha s_{4} \hat{\rho}-q^{-n} t-q^{n} \alpha^{2} s_{4}^{2} t^{-1}\right] .
\end{aligned}
$$


In order to proceed any further, we will require representations of the individual evaluated spectral coefficients which we give as two distinct partial fraction expansions. We offer these without proofs as they are the outcome of straightforward (though lengthy) computations.

LEMma 7.3. The evaluated spectral coefficients possess the rational function forms in the $f, l$ variables

$$
\begin{aligned}
& 2 t[n]\left[n+\frac{1}{2}\right] \hat{a}_{n} \frac{\mathfrak{W}_{+}\left(z_{5} ; q^{1 / 2} t\right)}{\mathfrak{T}_{+}\left(z_{5} ; q^{1 / 2} t\right)} \\
& =-q^{-1} \hat{f}^{-2}(q t-\alpha \hat{f})\left(q^{n} \alpha t \tilde{\sigma}_{6}-q^{-n} \hat{f}\right) \frac{w(\hat{f})}{(\hat{f}-\hat{l})\left(\hat{f}-\hat{l}^{-1}\right)} \\
& +\alpha^{-1} \tilde{\sigma}_{6} \hat{f}^{-1}\left(\alpha t-q^{-n} \hat{f}\right)\left(q^{n} \alpha t-\hat{f}\right)\left(\hat{l}+\hat{l}^{-1}\right) \\
& +\alpha^{-1} \hat{f}^{-2}\left(q^{n} \alpha t-\hat{f}\right)\left[-q^{-n} \tilde{\sigma}_{6}\left(\hat{f}^{2}-1\right)\right. \\
& \times\left(q^{n} \alpha t+\hat{f}\right)-q^{-1 / 2} \alpha t \tilde{\sigma}_{6} \sigma_{1} \hat{f} \\
& +q^{-n-5 / 2} \alpha^{2} \sigma_{3} \hat{f}^{2}+\left(q^{-n+2}+q^{n-2} \alpha^{4} \sigma_{4}\right) \\
& \left.\times \hat{f}\left(-q^{-n-2}+q^{-4} \alpha t \sigma_{4} \hat{f}\right)\right], \\
& 2 t[n]\left[n+\frac{1}{2}\right] \check{a}_{n} \frac{\mathfrak{W}_{+}\left(z_{6} ; q^{-1 / 2} t\right)}{\mathfrak{T}_{+}\left(z_{6} ; q^{-1 / 2} t\right)} \\
& =-q^{-1} \check{f}^{-2} \frac{(t-\alpha \check{f})(\alpha t-q \check{f})\left(q^{n} \alpha \tilde{\sigma}_{6}-q^{-n} t \check{f}\right)}{(\alpha-t \check{f})} \frac{w(\check{f})}{(\check{f}-\check{l})\left(\check{f}-\check{l}^{-1}\right)} \\
& +\alpha^{-1} \tilde{\sigma}_{6} \check{f}^{-1}\left(\alpha t-q^{-n+1} \check{f}\right)\left(q^{n-1} \alpha t-\check{f}\right)\left(\check{l}+\check{l}^{-1}\right) \\
& -\frac{[n]\left(q-t^{2}\right) t^{3}}{q} \frac{(t-\alpha \check{f})}{(\alpha-t \check{f})} \frac{w\left(\alpha t^{-1}\right)}{(\alpha-t \check{l})\left(\alpha-t \check{l}^{-1}\right)} \\
& +\alpha^{-1} t^{-1} \check{f}^{-2}\left(q^{n-1} \alpha t-\check{f}\right)\left[-q^{-n} t \tilde{\sigma}_{6}\left(\check{f}^{2}-1\right)\right. \\
& \times\left(q^{n} \alpha t+q \check{f}\right)-q^{-n}\left(q-t^{2}\right) \tilde{\sigma}_{6} \check{f}\left(q^{n} t+\alpha \check{f}\right) \\
& +q^{-2-2 n} t^{2} \check{f}\left(-q^{2} t+q^{n} \alpha \sigma_{4} \check{f}\right)-q^{-1 / 2} \alpha t^{2} \tilde{\sigma}_{6} \sigma_{1} \check{f} \\
& \left.+q^{-\frac{3}{2}-n} \alpha^{2} t \sigma_{3} \check{f}^{2}-\alpha^{2} \tilde{\sigma}_{6} \check{f}\left(t-q^{n-2} \alpha \sigma_{4} \check{f}\right)\right], \\
& 2 t[n]\left[n+\frac{1}{2}\right] \hat{a}_{n} \frac{\mathfrak{W}_{-}\left(E_{u}^{2+} z_{6} ; q^{1 / 2} t\right)}{\mathfrak{T}_{+}\left(E_{u}^{2+} z_{6} ; q^{1 / 2} t\right)}
\end{aligned}
$$




$$
\begin{aligned}
& =q^{-1 / 2} \hat{f}^{-2}(\alpha t-\hat{f})\left(q^{n+1 / 2} t \tilde{\sigma}_{6}-q^{-n-1 / 2} \alpha \hat{f}\right) \\
& \times \frac{w(\hat{f})}{(\hat{f}-\hat{l})\left(\hat{f}-\hat{l}^{-1}\right)}-\alpha^{-1} \tilde{\sigma}_{6} \hat{f}^{-1}\left(\alpha t-q^{-n} \hat{f}\right) \\
& \times\left(q^{n} \alpha t-\hat{f}\right)\left(\hat{l}+\hat{l}^{-1}\right) \\
& +\hat{f}^{-2}\left(q^{n} \alpha t-\hat{f}\right)\left[q^{-3-n}\left(q^{n}-1\right) \alpha \sigma_{4} \hat{f}(1+\alpha t \hat{f})\right. \\
& +q^{-1 / 2} t \tilde{\sigma}_{6} \sigma_{1} \hat{f}-q^{-n-5 / 2} \alpha \sigma_{3} \hat{f}^{2} \\
& \left.-\left(q^{n+1 / 2} t \tilde{\sigma}_{6}-q^{-n-1 / 2} \alpha \hat{f}\right)\left(q^{-n-1 / 2}+q^{-5 / 2} \sigma_{4} \hat{f}^{2}\right)\right], \\
& 2 t[n]\left[n+\frac{1}{2}\right] \check{a}_{n} \frac{\mathfrak{W}_{-}\left(E_{u}^{2-} z_{5} ; q^{-1 / 2} t\right)}{\mathfrak{T}_{+}\left(E_{u}^{2-} z_{5} ; q^{-1 / 2} t\right)} \\
& =q^{-1 / 2} \check{f}^{-2} \frac{(t-\alpha \check{f})(\alpha t-q \check{f})\left(q^{n+1 / 2} \tilde{\sigma}_{6}-q^{-n-1 / 2} \alpha t \check{f}\right)}{(q-\alpha t \check{f})} \\
& \times \frac{w(\check{f})}{(\check{f}-\check{l})\left(\check{f}-\check{l}^{-1}\right)} \\
& -\alpha^{-1} \tilde{\sigma}_{6} \check{f}^{-1}\left(\alpha t-q^{-n+1} \check{f}\right)\left(q^{n-1} \alpha t-\check{f}\right)\left(\check{l}+\check{l}^{-1}\right) \\
& +\frac{[n]\left(q-t^{2}\right) \alpha^{4} t^{3}}{q^{2}} \frac{(\alpha t-q \check{f})}{(q-\alpha t \check{f})} \frac{w\left(q \alpha^{-1} t^{-1}\right)}{(q-\alpha t \check{l})\left(q-\alpha t \check{l}^{-1}\right)} \\
& +t^{-1} \check{f}^{-2}\left(q^{n-1} \alpha t-\check{f}\right)\left[q^{1-n} \alpha^{-1} t \tilde{\sigma}_{6}\left(\check{f}^{2}-1\right)\left(q^{n-1} \alpha t+\check{f}\right)\right. \\
& +q^{-4-n}\left(q-t^{2}\right) \sigma_{4} \check{f}\left(q^{n} \alpha^{3} t+q^{2} \check{f}\right) \\
& +q^{-1 / 2} t^{2} \tilde{\sigma}_{6} \sigma_{1} \check{f}-q^{-n-3 / 2} \alpha t \sigma_{3} \check{f}^{2} \\
& \left.-q^{-4-2 n} \alpha\left(q t^{2}+q^{2 n} \sigma_{4}\right) \check{f}\left(-q^{2} t+q^{n} \alpha \sigma_{4} \check{f}\right)\right] .
\end{aligned}
$$

In addition, the evaluations at the advanced coordinate have alternative forms.

LEMmA 7.4. The advanced evaluated spectral coefficients are rational functions in the $g, f$ variables

$$
\begin{aligned}
& 2 t[n]\left[n+\frac{1}{2}\right] \hat{a}_{n} \frac{\mathfrak{W}_{+}\left(z_{5} ; q^{1 / 2} t\right)}{\mathfrak{T}_{+}\left(z_{5} ; q^{1 / 2} t\right)} \\
& \quad=-q^{-n-1} \alpha s_{4} \frac{\left(q^{n} t \alpha s_{4} \hat{g}^{-1}-1\right)^{2}}{\hat{g}-\hat{g}^{-1}} \frac{w\left(s_{4}{ }^{-1} \hat{g}\right)}{\hat{f}-s_{4}{ }^{-1} \hat{g}}
\end{aligned}
$$




$$
\begin{aligned}
& +q^{-n-1} \alpha s_{4} \frac{\left(q^{n} t \alpha s_{4} \hat{g}-1\right)^{2}}{\hat{g}-\hat{g}^{-1}} \frac{w\left(s_{4}^{-1} \hat{g}^{-1}\right)}{\hat{f}-s_{4}^{-1} \hat{g}^{-1}} \\
& -q^{-n-1} \alpha\left(\hat{g}^{2}+\hat{g}^{-2}\right)-q^{-n-6} s_{4}{ }^{-1} \\
& \times\left[-q^{7 / 2} \alpha \sigma_{3}+t \sigma_{4}\left(q^{4}-2 q^{n+3} \alpha^{2}+q^{2 n} \alpha^{4} \sigma_{4}\right)\right]\left(\hat{g}+\hat{g}^{-1}\right) \\
& +q^{-2 n-6} \alpha^{-1}\left[q^{6}+t^{2} q^{4 n} \alpha^{6} \sigma_{4}^{2}\right. \\
& -t^{2} q^{3 n+1} \alpha^{4} \sigma_{4}\left(q^{2}+\sigma_{4}\right)+q^{2 n+2} \alpha^{2}\left(t^{2} q^{2}+\alpha^{2}\right) \sigma_{4} \\
& \left.-q^{n+4} \alpha^{2}\left(2 q+\sigma_{2}\right)+q^{2 n+5 / 2} \alpha^{3} t\left(-q \sigma_{3}+\sigma_{1} \sigma_{4}\right)\right]
\end{aligned}
$$

and

$$
\begin{aligned}
2 t[n][ & \left.n+\frac{1}{2}\right] \hat{a}_{n} \frac{\mathfrak{W}_{-}\left(E_{u}^{2+} z_{6} ; q^{1 / 2} t\right)}{\mathfrak{T}_{+}\left(E_{u}^{2+} z_{6} ; q^{1 / 2} t\right)} \\
= & q^{-n-1} \alpha s_{4} \frac{\left(q^{n} t \alpha s_{4} \hat{g}^{-1}-1\right)^{2}}{\hat{g}-\hat{g}^{-1}} \frac{w\left(s_{4}{ }^{-1} \hat{g}\right)}{\hat{f}-s_{4}{ }^{-1} \hat{g}} \\
& -q^{-n-1} \alpha s_{4} \frac{\left(q^{n} t \alpha s_{4} \hat{g}-1\right)^{2}}{\hat{g}-\hat{g}^{-1}} \frac{w\left(s_{4}^{-1} \hat{g}^{-1}\right)}{\hat{f}-s_{4}^{-1} \hat{g}^{-1}} \\
& +q^{-n-1} \alpha\left(\hat{g}^{2}+\hat{g}^{-2}\right)-q^{-n-15 / 2} \alpha s_{4}^{-1} \\
& \times\left[q^{5} \sigma_{3}-t \alpha \sigma_{4}\left(q^{9 / 2}-2 q^{n+9 / 2}+q^{2 n+5 / 2} \sigma_{4}\right)\right]\left(\hat{g}+\hat{g}^{-1}\right) \\
& +q^{-2 n-11 / 2} \alpha\left[-q^{9 / 2}-q^{4 n+1 / 2} \alpha^{2} t^{2} \sigma_{4}^{2}\right. \\
& +q^{3 n+1 / 2} \alpha^{2} t^{2} \sigma_{4}\left(q^{2}+\sigma_{4}\right)-q^{2 n+5 / 2}\left(1+\alpha^{2} t^{2}\right) \sigma_{4} \\
& \left.+q^{n+7 / 2}\left(2 q+\sigma_{2}\right)-q^{2 n+2} \alpha t\left(-q \sigma_{3}+\sigma_{1} \sigma_{4}\right)\right] .
\end{aligned}
$$

At this stage, we have accumulated enough results to deduce the dynamical equations for our system on the deformation lattice.

Proposition 7.9. The t-evolution of our system in the variables $\rho, \lambda, f$ is given by a pair of coupled first-order equations, the first of which is

$$
\begin{gathered}
\frac{2 q s_{4} \hat{\rho}-t^{2} \check{f}^{-1}-q^{2} t^{-2} s_{4}^{2} \check{f}}{2 s_{4} \check{\rho}-\check{f}^{-1}-s_{4}^{2} \check{f}} \\
=\frac{q(t-\alpha \check{f})(q \check{f}-\alpha t)}{(t \check{f}-\alpha)(q-\alpha t \check{f})}
\end{gathered}
$$




$$
\begin{aligned}
& +\frac{\alpha^{4} t^{2}\left(q-t^{2}\right)}{\left(q-\alpha^{2}\right)} \frac{(q \check{f}-\alpha t)}{(q-\alpha t \check{f})} \\
& \times \frac{\check{f}^{2} w\left(q \alpha^{-1} t^{-1}\right)}{q \alpha t w(\check{f})-(q \check{f}-\alpha t)(q-\alpha t \check{f}) \check{f}\left[2 s_{4} \check{\rho}-\check{f}-1-s_{4}^{2} \check{f}\right]} \\
& -\frac{q t^{2}\left(q-t^{2}\right)}{\left(q-\alpha^{2}\right)} \frac{(t-\alpha \check{f})}{(t \check{f}-\alpha)} \\
& \times \frac{\check{f}^{2} w\left(\alpha t^{-1}\right)}{\alpha t w(\check{f})-(t \check{f}-\alpha)(t-\alpha \check{f}) \check{f}\left[2 s_{4} \check{\rho}-\check{f}-1-s_{4}^{2} \check{f}\right]} .
\end{aligned}
$$

The auxiliary equation for the leading coefficient of the polynomial is

$$
t^{2} \frac{\hat{\gamma}_{n}^{2}}{\check{\gamma}_{n}^{2}}=\frac{t^{2}+q^{2 n} \alpha^{2} s_{4}^{2}-2 q^{n} \alpha t s_{4} \hat{\rho}}{1+q^{2 n} \alpha^{2} t^{2} s_{4}^{2}-2 q^{n} \alpha t s_{4} \hat{\rho}} .
$$

Proof. The first of these equations is derived utilizing the following steps. We substitute the expression for $r_{1+} \check{a}_{n} / p_{+}$given by (7.112) and the transformation formulas for $\check{\mathfrak{z}}_{ \pm}$as given in (7.110) and (7.111) into the solution at the retarded coordinate (7.109). This yields an equation involving $\hat{\rho}$ on the one hand, and $\check{l}, \check{f}$ on the other hand. Now we perform a partial fraction expansion of this with respect to $\check{l}$, and this produces the following expression

$$
\begin{aligned}
2 s_{4} \hat{\rho}= & q^{-1} t^{2} \check{f}^{-1}+q t^{-2} s_{4}^{2} \check{f} \\
& -\frac{(t-\alpha \check{f})(q \check{f}-\alpha t)}{\check{f}(t \check{f}-\alpha)(q-\alpha t \check{f})} \frac{w(\check{f})}{\check{f}^{2}+1-2 \check{f} \check{\lambda}} \\
& -\frac{\alpha^{4} t^{2}\left(q-t^{2}\right)(q \check{f}-\alpha t)}{q\left(q-\alpha^{2}\right)(q-\alpha t \check{f})} \frac{w\left(q \alpha^{-1} t^{-1}\right)}{q^{2}+\alpha^{2} t^{2}-2 q \alpha t \check{\lambda}} \\
& +\frac{t^{2}\left(q-t^{2}\right)(t-\alpha \check{f})}{\left(q-\alpha^{2}\right)(t \check{f}-\alpha)} \frac{w\left(\alpha t^{-1}\right)}{\alpha^{2}+t^{2}-2 \alpha t \check{\lambda}},
\end{aligned}
$$

which is a simple function of $\check{\lambda}$. Then one substitutes for $\check{\lambda}$ using the inversion of the transformation (7.114) at the retarded time, and the result is (7.134). Alternatively, one can prove this formula by substituting (7.129) and (7.131) into (7.127).

We employ the variable transformation (7.112) and note that the lefthand side of this expression has been evaluated in (7.81) and (7.83). Equating these two forms gives (7.135). 
REMARK 7.8. The right-hand side of formula (7.134) exhibits apparent poles at $\alpha=t \check{f}$ and $q=\alpha t \check{f}$. However, this is not the case, as the former is cancelled by opposing contributions from the first and third terms, while the latter is cancelled by contributions from the first and second terms.

Clearly (7.134) is not manifestly invertible for $\check{\rho}$, however it is possible to construct a linear equation for the retarded variables by switching from $\rho$ to $\lambda$.

Proposition 7.10. The inverse to ( $\% .134)$ is given as a relation for $\check{\lambda}$, which we give in two alternative forms

$$
\begin{aligned}
\check{\lambda}= & \frac{\left(\hat{f}-q^{n} \alpha t\right)\left(t-q^{n} \alpha s_{4}^{2} \hat{f}\right)}{\left(t \hat{f}-q^{n} \alpha\right)\left(1-q^{n} \alpha t s_{4}^{2} \hat{f}\right)}\left[\hat{\lambda}-\frac{1}{2}\left(\hat{f}+\hat{f}^{-1}\right)\right]+\frac{t^{4}+\hat{f}^{2}}{2 t^{2} \hat{f}} \\
& +\frac{t^{2}\left(t^{2}-1\right) s_{4}^{3}}{2 q^{n+1 / 2}\left[n+\frac{1}{2}\right]} \\
& \times\left[-s_{4}^{3} \frac{\left(\hat{f}-q^{n} \alpha t\right)}{\left(1-q^{n} \alpha t s_{4}^{2} \hat{f}\right)} \frac{q^{4 n} \alpha^{4} w\left(q^{-n} \alpha^{-1} t^{-1} s_{4}^{-2}\right)}{1+q^{2 n} \alpha^{2} t^{2} s_{4}^{2}-2 q^{n} \alpha t s_{4} \hat{\rho}}\right. \\
& \left.+s_{4}^{-3} \frac{\left(t-q^{n} \alpha s_{4}^{2} \hat{f}\right)}{\left(t \hat{f}-q^{n} \alpha\right)} \frac{w\left(q^{n} \alpha t^{-1}\right)}{t^{2}+q^{2 n} \alpha^{2} s_{4}^{2}-2 q^{n} \alpha t s_{4} \hat{\rho}}\right]
\end{aligned}
$$

or

$$
\begin{aligned}
\check{\lambda}= & -\frac{\left(\hat{g}-q^{n} \alpha t s_{4}\right)\left(t-q^{n} \alpha s_{4} \hat{g}\right)}{2\left(\hat{g}^{2}-1\right)\left(1-q^{n} \alpha t s_{4} \hat{g}\right)\left(t \hat{g}-q^{n} \alpha s_{4}\right)} \frac{w\left(s_{4}^{-1} \hat{g}\right)}{\hat{f}-s_{4}^{-1} \hat{g}} \\
& +\frac{\hat{g}^{2}\left(1-q^{n} \alpha t s_{4} \hat{g}\right)\left(t \hat{g}-q^{n} \alpha s_{4}\right)}{2\left(\hat{g}^{2}-1\right)\left(\hat{g}-q^{n} \alpha t s_{4}\right)\left(t-q^{n} \alpha s_{4} \hat{g}\right)} \frac{w\left(s_{4}^{-1} \hat{g}^{-1}\right)}{\hat{f}-s_{4}^{-1} \hat{g}^{-1}} \\
& +\frac{1}{2 \alpha t^{3} \sigma_{4}}\left[q^{1 / 2} \alpha t \sigma_{3}+q^{5 / 2}\left(t^{2}-1\right)\left\{n+\frac{1}{2}\right\}\right]-\frac{1}{2 t^{2} s_{4}}\left[\hat{g}+\hat{g}^{-1}\right] \\
& +\frac{\alpha^{2} t\left(t^{2}-1\right) s_{4}^{3}\left[q^{2 n} \alpha s_{4} \frac{w\left(q^{-n} \alpha^{-1} t^{-1} s_{4}^{-2}\right)}{2 q^{1 / 2}\left[n+\frac{1}{2}\right]}\left(1-q^{n} \alpha t s_{4} \hat{g}\right)\left(1-q^{n} \alpha t s_{4} \hat{g}^{-1}\right)\right.}{w\left(q^{n} \alpha t^{-1}\right)} \\
& \left.-\alpha^{-1} s_{4}^{-1} \frac{\left.q^{n} \alpha s_{4} \hat{g}\right)\left(t-q^{n} \alpha s_{4} \hat{g}^{-1}\right)}{\left(t-q^{2}\right.}\right] .
\end{aligned}
$$


Proof. One can solve the compatibility relation (6.67) for the $(1,2)$ component of $A_{n}^{*}\left(z ; q^{-1 / 2} t\right)$ and find $\mathfrak{T}_{+}\left(z ; q^{-1 / 2} t\right)$ :

$$
\begin{aligned}
\mathfrak{T}_{+}\left(z ; q^{-1 / 2} t\right)= & \frac{1}{\chi(z, t)} \frac{\check{a}_{n} \hat{a}_{n}}{4 H_{n}^{2}(R+\Delta v S)\left(q^{1 / 2} z ; t\right)(R-\Delta v S)\left(q^{1 / 2} z ; t\right)} \\
& \times\left[\mathfrak{R}_{-}\left(q^{1 / 2} z ; t\right) \mathfrak{R}_{-}\left(q^{-1 / 2} z ; t\right) \mathfrak{T}_{+}\left(z ; q^{1 / 2} t\right)\right. \\
& +\mathfrak{P}_{+}\left(q^{1 / 2} z ; t\right) \mathfrak{P}_{+}\left(q^{-1 / 2} z ; t\right) \mathfrak{T}_{-}\left(z ; q^{1 / 2} t\right) \\
& +\mathfrak{R}_{-}\left(q^{1 / 2} z ; t\right) \mathfrak{P}_{+}\left(q^{-1 / 2} z ; t\right) \mathfrak{W}_{+}\left(z ; q^{1 / 2} t\right) \\
& \left.-\mathfrak{P}_{+}\left(q^{1 / 2} z ; t\right) \mathfrak{R}_{-}\left(q^{-1 / 2} z ; t\right) \mathfrak{W}_{-}\left(z ; q^{1 / 2} t\right)\right] .
\end{aligned}
$$

To simplify the calculations of the spectral matrix elements, we employ an alternative parameterization to that of (7.57), (7.58), and (7.59):

$$
\begin{aligned}
\mathfrak{W}_{+}( & \left.; q^{1 / 2} t\right) \\
= & -\frac{\alpha t\left(z^{2}-1\right)(q t-\alpha z)\left(q^{2 n+1} t \tilde{\sigma}_{6} z-\alpha\right)}{z^{2}\left(1-\alpha^{2} t^{2}\right)\left(q-\alpha^{2}\right)\left(q^{2 n+1} t^{2} \tilde{\sigma}_{6}-1\right)} \mathfrak{W}_{+}\left(\alpha t ; q^{1 / 2} t\right) \\
& +\frac{q^{2} \alpha t\left(z^{2}-1\right)(z-\alpha t)\left(q^{2 n} \alpha t \tilde{\sigma}_{6} z-1\right)}{z^{2}\left(q^{2} t^{2}-\alpha^{2}\right)\left(q-\alpha^{2}\right)\left(q^{2 n+1} t^{2} \tilde{\sigma}_{6}-1\right)} \mathfrak{W}_{-}\left(q^{-1} \alpha t^{-1} ; q^{1 / 2} t\right) \\
& +\frac{(z+1)(z-\alpha t)(q t-\alpha z)\left(q^{2}-q^{2 n} \alpha^{2} t^{2} \sigma_{4} z\right)}{2 q t z^{2}\left(q^{2}-q^{2 n} \alpha^{2} t^{2} \sigma_{4}\right)} w(1) \\
& -\frac{(z-1)(z-\alpha t)(q t-\alpha z)\left(q^{2}+q^{2 n} \alpha^{2} t^{2} \sigma_{4} z\right)}{2 q t z^{2}\left(q^{2}-q^{2 n} \alpha^{2} t^{2} \sigma_{4}\right)} w(-1) \\
& +\frac{q^{-n-3}\left(z^{2}-1\right)(z-\alpha t)(q t-\alpha z)}{\alpha t^{2} z^{3}\left(q^{2}-q^{2 n} \alpha^{2} t^{2} \sigma_{4}\right)} \\
& \times\left[q^{4}+q^{4 n} \alpha^{4} t^{4} \sigma_{4}^{2} z^{2}\right. \\
& \left.+q^{2 n+3 / 2} \alpha^{2} t^{2}\left(q \sigma_{3} z+\sigma_{1} \sigma_{4} z-q^{1 / 2}\left(1+z^{2}\right)\right)\right] \\
\mathfrak{W}_{-} & \left(z ; q^{1 / 2} t\right) \\
= & \frac{\alpha t\left(z^{2}-1\right)(q t z-\alpha)\left(q^{2 n+1} t \tilde{\sigma}_{6}-\alpha z\right)}{z^{2}\left(1-\alpha^{2} t^{2}\right)\left(q-\alpha^{2}\right)\left(q^{2 n+1} t^{2} \tilde{\sigma}_{6}-1\right)} \mathfrak{W}_{+}\left(\alpha t ; q^{1 / 2} t\right) \\
& +\frac{q^{2} \alpha t\left(z^{2}-1\right)(1-\alpha t z)\left(z-q^{2 n} \alpha t \tilde{\sigma}_{6}\right)}{z^{2}\left(q^{2} t^{2}-\alpha^{2}\right)\left(q-\alpha^{2}\right)\left(q^{2 n+1} t^{2} \tilde{\sigma}_{6}-1\right)} \mathfrak{W}_{-}\left(q^{-1} \alpha t^{-1} ; q^{1 / 2} t\right)
\end{aligned}
$$


$(7.141)$

$$
\begin{aligned}
& +\frac{(z+1)(1-\alpha t z)(q t z-\alpha)\left(q^{2} z-q^{2 n} \alpha^{2} t^{2} \sigma_{4}\right)}{2 q t z^{2}\left(q^{2}-q^{2 n} \alpha^{2} t^{2} \sigma_{4}\right)} w(1) \\
& +\frac{(z-1)(1-\alpha t z)(q t z-\alpha)\left(q^{2} z+q^{2 n} \alpha^{2} t^{2} \sigma_{4}\right)}{2 q t z^{2}\left(q^{2}-q^{2 n} \alpha^{2} t^{2} \sigma_{4}\right)} w(-1) \\
& -\frac{q^{-n-3}\left(z^{2}-1\right)(1-\alpha t z)(q t z-\alpha)}{\alpha t^{2} z^{3}\left(q^{2}-q^{2 n} \alpha^{2} t^{2} \sigma_{4}\right)} \\
& \times\left[q^{4} z^{2}+q^{4 n} \alpha^{4} t^{4} \sigma_{4}^{2}\right. \\
& \left.+q^{2 n+3 / 2} \alpha^{2} t^{2}\left(q \sigma_{3} z+\sigma_{1} \sigma_{4} z-q^{1 / 2}\left(1+z^{2}\right)\right)\right]
\end{aligned}
$$

and

$$
\begin{aligned}
& \mathfrak{T}_{+}\left(z ; q^{1 / 2} t\right) \\
& \quad=-\frac{\alpha t\left(z-z^{-1}\right)}{\left(q-\alpha^{2}\right)\left(q t^{2}-1\right)} p_{+}
\end{aligned}
$$

$$
\begin{aligned}
& \times\left[\frac{(q t z-\alpha)\left(q t z^{-1}-\alpha\right)}{\left(\alpha^{2} t^{2}-1\right)} \frac{\mathfrak{W}_{+}\left(\alpha t ; q^{1 / 2} t\right)}{r_{1,-\frac{1}{2}}\left(q^{-1 / 2} \alpha t+q^{1 / 2} \alpha^{-1} t^{-1}\right)+r_{0,-}}\right. \\
& \left.+q^{2} \frac{(z-\alpha t)\left(z^{-1}-\alpha t\right)}{\left(\alpha^{2}-q^{2} t^{2}\right)} \frac{\mathfrak{W}_{-}\left(q^{-1} \alpha t^{-1} ; q^{1 / 2} t\right)}{r_{1,-} \frac{1}{2}\left(q^{-1 / 2} \alpha t^{-1}+q^{1 / 2} \alpha^{-1} t\right)+r_{0,-}}\right],
\end{aligned}
$$

$$
\begin{aligned}
\mathfrak{T}_{-}\left(z ; q^{1 / 2} t\right) & \\
= & \frac{\alpha t\left(z-z^{-1}\right)}{\left(q-\alpha^{2}\right)\left(q t^{2}-1\right)} \frac{1}{p_{+}} \\
& \times\left[\frac{(q t z-\alpha)\left(q t z^{-1}-\alpha\right)}{\left(\alpha^{2} t^{2}-1\right)}\right.
\end{aligned}
$$

$$
\begin{aligned}
& \times\left(r_{1,-} \frac{1}{2}\left(q^{-1 / 2} \alpha t+q^{1 / 2} \alpha^{-1} t^{-1}\right)+r_{0,-}\right) \mathfrak{W}_{-}\left(\alpha t ; q^{1 / 2} t\right) \\
& +q^{2} \frac{(z-\alpha t)\left(z^{-1}-\alpha t\right)}{\left(\alpha^{2}-q^{2} t^{2}\right)} \\
& \times\left(r_{1,-} \frac{1}{2}\left(q^{-1 / 2} \alpha t^{-1}+q^{1 / 2} \alpha^{-1} t\right)+r_{0,-}\right) \\
& \left.\times \mathfrak{W}_{+}\left(q^{-1} \alpha t^{-1} ; q^{1 / 2} t\right)\right]
\end{aligned}
$$


This particular parameterization implies that the numerator of the righthand side of (7.139) manifestly contains a factor of $\left(z-z^{-1}\right)(q t z-\alpha)\left(q t z^{-1}-\right.$ $\alpha)(z-\alpha t)\left(z^{-1}-\alpha t\right)$ which is present in the denominator. This ensures that the ratio is linear in $x$, as it must. Finding the zero of this linear polynomial then gives $\check{\lambda}$, which after further substantial manipulation and simplification yields (7.138) and (7.137).

A crucial fact enabling further progress is the following factorization formula for a quantity that will subsequently figure prominently in certain discriminants.

Lemma 7.5. The biquadratic in $\hat{\rho}, \grave{\lambda}$

$$
\begin{aligned}
16 \sigma_{4}\left(\hat{\rho}^{2} \check{\lambda}^{2}-\hat{\rho}^{2}-\check{\lambda}^{2}\right)-8 s_{4}\left(q^{2}+q \sigma_{2}+\sigma_{4}\right) \hat{\rho} \check{\lambda} \\
\quad+8 q^{1 / 2} s_{4}\left(q \sigma_{1}+\sigma_{3}\right) \hat{\rho} \\
\quad+8 q^{-1 / 2}\left(\sigma_{1} \sigma_{4}+q \sigma_{3}\right) \check{\lambda}+\left(q-\sigma_{2}\right)^{2} \\
\quad-4 \sigma_{1} \sigma_{3}+2 \sigma_{4}-2 q^{-1} \sigma_{2} \sigma_{4}+q^{-2} \sigma_{4}^{2}
\end{aligned}
$$

is a perfect square which can be given in either of two ways. In the first way this is the square of

$$
\begin{aligned}
q t^{2} & \hat{f}^{-2}-q^{1 / 2} t^{2} \sigma_{1} \hat{f}^{-1}+q^{-1 / 2} t^{-2} \sigma_{3} \hat{f} \\
& -q^{-1} t^{-2} \sigma_{4} \hat{f}^{2}+q t^{-2}-q^{-1} t^{2} \sigma_{4} \\
& +q^{3 / 2}\left\{n+\frac{1}{2}\right\} \alpha^{-1} t^{-3}\left(t^{2}-1\right)\left(\hat{f}+t^{4} \hat{f}^{-1}\right) \\
& -2 q s_{4}\left(t^{-2} \hat{f}-t^{2} \hat{f}^{-1}\right) \hat{\rho} \\
& -q \frac{\left(1-s_{4}^{2} \hat{f}^{2}\right)\left(\hat{f}-q^{n} \alpha t\right)\left(t-q^{n} \alpha s_{4}^{2} \hat{f}\right)}{\hat{f}^{2}\left(t \hat{f}-q^{n} \alpha\right)\left(1-q^{n} \alpha t s_{4}^{2} \hat{f}\right)} \frac{w(\hat{f})}{1+s_{4}^{2} \hat{f}^{2}-2 s_{4} \hat{f} \hat{\rho}} \\
& +\frac{q^{1 / 2} \alpha t\left(t^{2}-1\right) s_{4}^{3}}{\left[n+\frac{1}{2}\right]} \\
& \times\left[\frac{q^{2 n} \alpha^{2} s_{4}^{3}\left(1-q^{2 n} \alpha^{2} t^{2} s_{4}^{2}\right)\left(\hat{f}-q^{n} \alpha t\right) w\left(q^{-n} \alpha^{-1} t^{-1} s_{4}^{-2}\right)}{\left(1-q^{n} \alpha t s_{4}^{2} \hat{f}\right)\left(1+q^{2 n} \alpha^{2} t^{2} s_{4}^{2}-2 q^{n} \alpha t s_{4} \hat{\rho}\right)}\right. \\
& \left.+\frac{q^{-2 n} \alpha^{-2} s_{4}^{-3}\left(t^{2}-q^{2 n} \alpha^{2} s_{4}^{2}\right)\left(t-q^{n} \alpha s_{4}^{2} \hat{f}\right) w\left(q^{n} \alpha t^{-1}\right)}{\left(t \hat{f}-q^{n} \alpha\right)\left(t^{2}+q^{2 n} \alpha^{2} s_{4}^{2}-2 q^{n} \alpha t s_{4} \hat{\rho}\right)}\right],
\end{aligned}
$$


and in the second way this is the square of

$$
\begin{aligned}
& q^{1 / 2} \sigma_{3} t^{-2} \check{f}-q^{-1 / 2} \sigma_{1} t^{2} \check{f}^{-1} \\
& -\left(t^{2} \check{f}^{-2}+q^{2} t^{-2} s_{4}^{2}\right)\left[\check{f}^{2}-1+q^{-1}\left(q+\alpha^{2}\right) \alpha^{-1} t^{-1}\left(q-t^{2}\right) \check{f}\right] \\
& +2 \check{\lambda}\left(t^{2} \check{f}^{-1}-q^{2} t^{-2} s_{4}^{2} \check{f}\right) \\
& +\frac{q\left(\check{f}^{2}-1\right)(q \check{f}-\alpha t)(t-\alpha \check{f})}{\check{f}^{2}(t \check{f}-\alpha)(q-\alpha t \check{f})} \frac{w(\check{f})}{f^{2}+1-2 \check{f} \check{\lambda}} \\
& +\frac{t\left(q-t^{2}\right)}{q \alpha\left(q-\alpha^{2}\right)}\left[\frac{t-\alpha \check{f}}{t \check{f}-\alpha} \frac{q^{2}\left(t^{2}-\alpha^{2}\right) w\left(\alpha t^{-1}\right)}{\alpha^{2}+t^{2}-2 \alpha t \check{\lambda}}\right. \\
& \left.+\frac{q \check{f}-\alpha t \alpha^{4}\left(q^{2}-\alpha^{2} t^{2}\right) w\left(q \alpha^{-1} t^{-1}\right)}{q-\alpha t \check{f}}\right] .
\end{aligned}
$$

Proof. In the first way we substitute (7.138) into (7.144), whereas in the second way we substitute (7.136) into (7.144). After considerable simplification we arrive at the two results.

We are now in a position to derive the evolution equations for the $f$ variable, first in the advanced direction.

Proposition 7.11. Assuming that $\check{f} \neq 0, t \check{f}-\alpha \neq 0$, and $q-\alpha t \check{f} \neq 0$, we have the forward evolution for $f$

$$
\begin{aligned}
\hat{f}= & \left\{\frac { 2 t ^ { 2 } ( t ^ { 2 } - 1 ) s _ { 4 } ^ { 2 } } { q ^ { n - 1 / 2 } [ n + \frac { 1 } { 2 } ] } \left[-\frac{q^{4 n} \alpha^{4} s_{4}^{4} w\left(q^{-n} \alpha^{-1} t^{-1} s_{4}^{-2}\right)}{1+q^{2 n} \alpha^{2} t^{2} s_{4}^{2}-2 q^{n} \alpha t s_{4} \hat{\rho}}\right.\right. \\
& \left.+\frac{w\left(q^{n} \alpha t^{-1}\right)}{t^{2}+q^{2 n} \alpha^{2} s_{4}^{2}-2 q^{n} \alpha t s_{4} \hat{\rho}}\right] \\
& -\sigma_{2}-q^{-1}\left(1-2 t^{2}\right) \sigma_{4}-q\left(1-2 t^{-2}\right) \\
& +2 \check{\lambda}\left[2 q s_{4} \hat{\rho}-t^{2} \check{f}^{-1}+q^{2} t^{-2} s_{4}^{2} \check{f}\right] \\
& -q^{1 / 2} \sigma_{3} t^{-2} \check{f}+q^{-1 / 2} \sigma_{1} t^{2} \check{f}^{-1} \\
& +\left(t^{2} \check{f}^{-2}+q^{2} t^{-2} s_{4}^{2}\right)\left[\check{f}^{2}-1+q^{-1}\left(q+\alpha^{2}\right) \alpha^{-1} t^{-1}\left(q-t^{2}\right) \check{f}\right] \\
& -\frac{q\left(\check{f}^{2}-1\right)(q \check{f}-\alpha t)(t-\alpha \check{f})}{\check{f}^{2}(t \check{f}-\alpha)(q-\alpha t \check{f})} \frac{w(\check{f})}{\check{f}^{2}+1-2 \check{f} \check{\lambda}} \\
& -\frac{t\left(q-t^{2}\right)}{q \alpha\left(q-\alpha^{2}\right)}\left[\frac{t-\alpha \check{f}}{t \check{f}-\alpha} \frac{q^{2}\left(t^{2}-\alpha^{2}\right) w\left(\alpha t^{-1}\right)}{\alpha^{2}+t^{2}-2 \alpha t \check{\lambda}}\right.
\end{aligned}
$$




$$
\begin{aligned}
& \left.\left.+\frac{q \check{f}-\alpha t}{q-\alpha t \check{f}} \frac{\alpha^{4}\left(q^{2}-\alpha^{2} t^{2}\right) w\left(q \alpha^{-1} t^{-1}\right)}{q^{2}+\alpha^{2} t^{2}-2 q \alpha t \check{\lambda}}\right]\right\} \\
& \times\left\{\frac { 2 q ^ { 1 / 2 } t ( t ^ { 2 } - 1 ) } { [ n + \frac { 1 } { 2 } ] } \left[-\frac{q^{2 n} \alpha^{3} s_{4}^{6} w\left(q^{-n} \alpha^{-1} t^{-1} s_{4}^{-2}\right)}{1+q^{2 n} \alpha^{2} t^{2} s_{4}^{2}-2 q^{n} \alpha t s_{4} \hat{\rho}}\right.\right. \\
& \left.+\frac{\alpha s_{4}^{4} w\left(q^{n} \alpha t^{-1}\right)}{t^{2}+q^{2 n} \alpha^{2} s_{4}^{2}-2 q^{n} \alpha t s_{4} \hat{\rho}}\right] \\
& -2 q^{3 / 2} \alpha^{-1} t^{-3}\left\{n+\frac{1}{2}\right\}\left(t^{2}-1\right) \\
& \left.-2 q^{-1} t^{-2}\left(q^{1 / 2} \sigma_{3}-2 t^{2} \sigma_{4} \check{\lambda}\right)+4 q t^{-2} s_{4} \hat{\rho}\right\}^{-1}
\end{aligned}
$$

where $\hat{\rho}$ is given by ( 7.134$)$.

Proof. While (7.138) can be primarily viewed as a linear equation for $\check{\lambda}$, it can also be viewed as a quadratic equation for $\hat{f}$, and, as such, possesses a discriminant which contains, apart from explicit squared factors, the factor (7.144). By substituting for $\hat{\rho}$ using (7.136), we can employ the result (7.146) and effect a factorization of the quadratic into linear factors. The choice of the factors can be settled by consideration of the known solution for $n=0$, and it transpires that the negative branch is appropriate. This yields (7.147).

Our last task is to derive the inverse to (7.147) and complete the system of evolution equations.

Proposition 7.12. The inverse of the evolution for $f$ is given by

$$
\begin{aligned}
\check{f}= & \left\{\frac{2 t^{2}\left(q-t^{2}\right)}{q-\alpha^{2}}\left[\frac{w\left(\alpha t^{-1}\right)}{\alpha^{2}+t^{2}-2 \alpha t \check{\lambda}}-q^{-1} \alpha^{4} \frac{w\left(q \alpha^{-1} t^{-1}\right)}{q^{2}+\alpha^{2} t^{2}-2 q \alpha t \check{\lambda}}\right]\right. \\
& +4 s_{4} \hat{\rho} \check{\lambda}+2 s_{4}\left(t^{-2} \hat{f}-t^{2} \hat{f}^{-1}\right) \hat{\rho}-t^{2} \hat{f}^{-2}+q^{-1 / 2} t^{2} \sigma_{1} \hat{f}^{-1} \\
& -q^{-1} \sigma_{2}-q^{-3 / 2} t^{-2} \sigma_{3} \hat{f}+q^{-2} t^{-2} \sigma_{4} \hat{f}^{2} \\
& -q^{-2} t^{-2}\left(-2 q+t^{2}-t^{4}\right) \sigma_{4}-q^{-1} t^{-2}\left(q\left(1+t^{2}\right)-2 t^{4}\right) \\
& -q^{1 / 2}\left\{n+\frac{1}{2}\right\} \alpha^{-1} t^{-3}\left(t^{2}-1\right)\left(\hat{f}+t^{4} \hat{f}^{-1}\right) \\
& +\frac{\left(1-s_{4}^{2} \hat{f}^{2}\right)\left(\hat{f}-q^{n} \alpha t\right)\left(t-q^{n} \alpha s_{4}^{2} \hat{f}\right)}{\hat{f}^{2}\left(t \hat{f}-q^{n} \alpha\right)\left(1-q^{n} \alpha t s_{4}^{2} \hat{f}\right)} \frac{w(\hat{f})}{1+s_{4}^{2} \hat{f}^{2}-2 s_{4} \hat{f} \hat{\rho}}
\end{aligned}
$$




$$
\begin{aligned}
& -\frac{\alpha t\left(t^{2}-1\right) s_{4}^{3}}{q^{1 / 2}\left[n+\frac{1}{2}\right]} \\
& \times\left[\frac{q^{2 n} \alpha^{2} s_{4}^{3}\left(1-q^{2 n} \alpha^{2} t^{2} s_{4}^{2}\right)\left(\hat{f}-q^{n} \alpha t\right) w\left(q^{-n} \alpha^{-1} t^{-1} s_{4}^{-2}\right)}{\left(1-q^{n} \alpha t s_{4}^{2} \hat{f}\right)\left(1+q^{2 n} \alpha^{2} t^{2} s_{4}^{2}-2 q^{n} \alpha t s_{4} \hat{\rho}\right)}\right. \\
& \left.\left.+\frac{q^{-2 n} \alpha^{-2} s_{4}^{-3}\left(t^{2}-q^{2 n} \alpha^{2} s_{4}^{2}\right)\left(t-q^{n} \alpha s_{4}^{2} \hat{f}\right) w\left(q^{n} \alpha t^{-1}\right)}{\left(t \hat{f}-q^{n} \alpha\right)\left(t^{2}+q^{2 n} \alpha^{2} s_{4}^{2}-2 q^{n} \alpha t s_{4} \hat{\rho}\right)}\right]\right\} \\
& \times\left\{\frac{2 \alpha t\left(q-t^{2}\right)}{q-\alpha^{2}}\left[\frac{w\left(\alpha t^{-1}\right)}{\alpha^{2}+t^{2}-2 \alpha t \check{\lambda}}-\frac{\alpha^{2} w\left(q \alpha^{-1} t^{-1}\right)}{q^{2}+\alpha^{2} t^{2}-2 q \alpha t \check{\lambda}}\right]\right. \\
& -2 q^{-1 / 2} t^{-2} \sigma_{3}+2 q^{-2} \alpha^{-1} t^{-3}\left(q-t^{2}\right)\left(q+\alpha^{2}\right) \sigma_{4} \\
& \left.+4 s_{4} \hat{\rho}+4 q^{-1} t^{-2} \sigma_{4} \check{\lambda}\right\}^{-1},
\end{aligned}
$$

where $\check{\lambda}$ is given by (\%.138).

Proof. In a similar manner to the previous proof we can solve (7.136) for $\check{f}$. On appearance this polynomial should be a quintic in $\check{f}$, however in line with Remark 7.8, we observe that this contains a factor of $\check{f}(t \check{f}-$ $\alpha)(q-\alpha t \check{f})$ and we only have to deal with a quadratic. Upon examining this quadratic, we find that the discriminant contains, again, the factor (7.144) as the only manifestly square-free factor. Upon substituting for $\check{\lambda}$ using (7.138), we can now employ (7.145) and factorize the quadratic. As in the previous case, consideration of the $n=0$ solution resolves the sign ambiguity in favor of the negative root, yielding (7.148).

\subsection{Seed solution}

We can now make contact with the earlier theory characterizing the moments, as given in Section 7.1, through a study of the classical "seed" solutions to the coupled recurrence system given above. We now append a subscript on the variables to indicate the $n$-value.

Proposition 7.13. The recurrence relations (7.134) and (7.147) admit the classical "seed" solution at $n=0$

$$
f_{0}(t)=q^{-1 / 2} \alpha t
$$

and

$$
\begin{aligned}
& 2 q s_{4} \rho_{0}(t) \\
& \qquad=\frac{C\left(q^{1 / 2} \alpha^{-1} t+\sigma_{4} q^{-1 / 2} \alpha t^{-1}\right) m(t)+D\left(q^{3 / 2} \alpha^{-1} t^{-1}+\sigma_{4} q^{-3 / 2} \alpha t\right) m\left(q^{-1} t\right)}{C m(t)+D m\left(q^{-1} t\right)},
\end{aligned}
$$


where $m(t)$ is the general solution to the second-order q-difference equation

$$
\begin{aligned}
& \left(t^{2}-q^{2}\right) t^{4} w\left(\alpha t^{-1}\right) m\left(q^{1 / 2} t\right) \\
& \quad+\frac{\hat{D}}{\hat{C}}\left[\left(t^{2}-q^{2}\right) t^{4} w\left(\alpha t^{-1}\right)\right. \\
& \quad+q^{3}\left(t^{2}-1\right) w\left(q^{-1} \alpha t\right)-q^{-3}\left(q-\alpha^{2}\right) \\
& \left.\quad \times\left(q^{2}-\sigma_{4} \alpha^{2}\right)\left(t^{2}-1\right)\left(t^{2}-q\right)\left(t^{2}-q^{2}\right)\right] m\left(q^{-1 / 2} t\right) \\
& \quad+\frac{\hat{D} \check{D}}{\hat{C} \check{C}} q^{3}\left(t^{2}-1\right) w\left(q^{-1} \alpha t\right) m\left(q^{-3 / 2} t\right)=0 .
\end{aligned}
$$

Proof. It is clear that both (7.130) and (7.131) vanish when (7.149) holds at $n=0$, therefore together they satisfy (7.117). Given (7.149), we observe that two terms vanish in (7.134) and that $\rho_{0}(t)$ satisfies the discrete Riccati equation

$$
\begin{aligned}
& 2 s_{4} \hat{\rho}_{0}-\alpha^{-1} t-\alpha t^{-1} s_{4}^{2} \\
& \quad=\frac{q t^{4} w\left(\alpha t^{-1}\right)\left[2 q s_{4} \check{\rho}_{0}-q^{2} \alpha^{-1} t^{-1}-\alpha t s_{4}^{2}\right]}{\alpha t\left(q-t^{2}\right)\left(q-\alpha^{2}\right)\left[2 q s_{4} \check{\rho}_{0}-q^{2} \alpha^{-1} t^{-1}-\alpha t s_{4}^{2}\right]+q^{4} w\left(q^{-1} \alpha t\right)} .
\end{aligned}
$$

Alternatively, we can derive this relation by specializing (7.137) under (7.149) which yields

$$
t^{2}+\alpha^{2}-2 \alpha t \check{\lambda}_{0}=\frac{t^{4} w\left(\alpha t^{-1}\right)}{t^{2}+\alpha^{2} s_{4}^{2}-2 \alpha t s_{4} \hat{\rho}_{0}},
$$

and then using (7.114) to substitute for $\check{\lambda}_{0}$. Making the standard linearizing transformation

$$
2 q s_{4} \rho_{0}(t)=\frac{A m(t)+B m\left(q^{-1} t\right)}{C m(t)+D m\left(q^{-1} t\right)},
$$

we find, in our solution for the decoupling factors, that $\hat{A}=\left(q \alpha^{-1} t+\sigma_{4} \times\right.$ $\left.q^{-1} \alpha t^{-1}\right) \hat{C}$ and $\check{B}=\left(q^{2} \alpha^{-1} t^{-1}+\sigma_{4} q^{-2} \alpha t\right) \check{D}$ and arrive at (7.151).

REMARK 7.9. Our explicit initial orthogonal polynomial variables are one specialization of the classical solutions given above. As remarked in the proof of Proposition 7.3, we have $\mu_{0}=V\left(\lambda_{0}\right)$ and $\nu_{0}=W\left(\lambda_{0}\right)$ at $n=0$, where $\lambda_{0}$ is the unique zero of the right-hand side of (7.18)

$$
2\left[\frac{1}{2}\right] \lambda_{0}=-q^{-1 / 2} \tilde{\sigma}_{1}+q^{1 / 2} \tilde{\sigma}_{5}-[1] \frac{m_{0,+}+m_{0,-}}{m_{0,0}} .
$$


We easily see that $\mathfrak{z}_{ \pm, 0}(t)=l_{0}^{\mp 3} \prod_{j=1}^{6}\left(1-q^{-1 / 2} a_{j} l_{0}^{ \pm 1}\right)$, and from the transformations (7.110) and (7.111) we deduce that $f_{0}(t)=q^{-1 / 2} \alpha t$. The elements of the spectral matrix $A_{0}^{*}$ are

$$
\begin{aligned}
\mathfrak{W}_{+, 0}(z ; t) & =z^{-3} w(z)\left(1-q^{-1 / 2} \alpha t z\right)\left(1-q^{-1 / 2} \alpha t^{-1} z\right) \\
& =W+\Delta y V \\
\mathfrak{W}_{-, 0}(z ; t) & =z^{3} w\left(z^{-1}\right)\left(1-q^{-1 / 2} \alpha t z^{-1}\right)\left(1-q^{-1 / 2} \alpha t^{-1} z^{-1}\right) \\
& =W-\Delta y V, \\
\mathfrak{T}_{+, 0}(z ; t) & =2 a_{0}\left[\frac{1}{2}\right]\left(z-z^{-1}\right)\left(z-l_{0}\right)\left(1-l_{0}^{-1} z^{-1}\right), \\
\mathfrak{T}_{-, 0}(z ; t) & =0,
\end{aligned}
$$

while those of the deformation matrix $B_{0}^{*}$ are

$$
\begin{aligned}
& \mathfrak{R}_{+, 0}(z ; t)=-\frac{a_{0} \hat{\gamma}_{0}}{\check{a}_{0} \check{\gamma}_{0}}\left(1-q^{-1 / 2} \alpha t z\right)\left(1-q^{-1 / 2} \alpha t z^{-1}\right), \\
& \mathfrak{R}_{-, 0}(z ; t)=-\frac{a_{0} \check{\gamma}_{0}}{\hat{a}_{0} \hat{\gamma}_{0}}\left(1-q^{-1 / 2} \alpha t^{-1} z\right)\left(1-q^{-1 / 2} \alpha t^{-1} z^{-1}\right), \\
& \mathfrak{P}_{+, 0}(z ; t)=2 q^{-1 / 2} \alpha a_{0}\left[t \frac{\hat{\gamma}_{0}}{\check{\check{\gamma}}_{0}}-t^{-1} \frac{\check{\gamma}_{0}}{\hat{\gamma}_{0}}\right], \\
& \mathfrak{P}_{-, 0}(z ; t)=0 .
\end{aligned}
$$

We also note from (7.135), (3.16), and (3.10) that

$$
2 \alpha s_{4} \rho_{0}(t)=\frac{\left(1+q^{-1} \alpha^{2} s_{4}^{2} t^{2}\right) m_{0,0}\left(q^{-1} t\right)-\left(1+q \alpha^{2} s_{4}^{2} t^{-2}\right) m_{0,0}(t)}{q^{-1 / 2} t m_{0,0}\left(q^{-1} t\right)-q^{1 / 2} t^{-1} m_{0,0}(t)}
$$

which is precisely the case of (7.150) with $D=-q^{-1} t^{2} C$. With this solution, we also deduce that the moment recurrence (7.78) for $m_{0,0}$ coincides with (7.151).

At the beginning of this section, in Remark 7.2, we noted that some explicit solutions to the moment recurrences were given in [97]. This knowledge enables the possibility of checking any aspect of the foregoing theoryeither symbolically or numerically — with essentially unlimited precision. We wish to report that extensive checking of all the key relations for the cases $n=0$ and $n=1$ has been carried out employing Mathematica code and 
utilities, where possible by exact symbolic means (or if not by numerical means) to a level of one part in $10^{20} \rightarrow 10^{30}$ or better at random exact values of the input parameters. These checks have also utilized a body of unreported work [96] covering the system of Laguerre-Freud recurrences under $n \mapsto n+1$ for general $n \in \mathbb{Z}_{\geq 0}$.

We now come to the question regarding the identity of this system in the Sakai scheme. While not providing an explicit transformation from our parameters and variables to those appearing in the canonical coupled $q$ difference system (see [33], [86]), we offer unambiguous evidence that it is one of the classical yet full parameter set cases of the $E_{7}^{(1)} q$-Painlevé system as can be seen from the following inspection of their classical solutions (see [71], [51], [52]), and the recent systematic study of two-Casorati determinantal forms of their classical solutions (see [69]). Clearly, the $\tau$-functions of our construction, given by (3.10) and (3.11), are of the two-Casorati determinant form because if we choose $b=a_{r}, a=a_{s}$ with $r, s \in\{1,2,3,4\}$, say, and this is employed in the definition (3.9), we have a moment determinant with elements $m_{0,0}\left(q^{j} a_{r}, q^{k} a_{s}\right), j, k \in \mathbb{Z}_{\geq 0}$, where $m_{0,0}$ is given by any $q$-constant linear combination of the two ${ }_{8} W_{7}$ solutions appearing in (7.9).

Several tasks that have arisen in the course of our study remain unfinished and we conclude by detailing them and the issues involved. Clarification of the explicit relationship of the evolution system in (7.134), (7.137), (7.147), and (7.148) with the canonical $q$-difference equations as, say, given in [51] and [52], is required. It remains to complete the construction of the $n \rightarrow$ $n+1$ recurrence relations which occupy a special place in our approach, but figure as Schlesinger transformations in the integrable theory. In fact our framework can easily treat the Schlesinger transformations of this example (or any other for that matter), and in particular the $n \rightarrow n+1$ recurrences, or the $a_{j}, \alpha \mapsto q a_{j}, q \alpha, j=1, \ldots, 4$ mappings. These latter transformations are manifested in our context as specialized Christoffel-Uvarov transformations, however we will postpone this undertaking.

Significant progress has been made in finding the analogue of an isomonodromic system for the elliptic Painlevé courtesy of a preprint by Eric Rains [85], and in the work by Yamada [98]. The approach taken in this former work is very much in the spirit of the present study, and it would be natural to expect that a limiting case of the results reported there would correspond our own. Very recently, Yamada [99] has given Lax pairs for the $E_{8}^{(1)}, E_{7}^{(1)}$, and $E_{6}^{(1)} q$-Painlevé equations by reformulating the $E_{8}^{(1)}$ elliptic Painlevé 
Lax pair system and taking limits $E_{8}^{(1)} \rightarrow E_{7}^{(1)} \rightarrow E_{6}^{(1)}$. One would expect that a gauge transformation and coordinate transformations would link our Lax pair with his.

Acknowledgments. This research has been supported by the Australian Research Council. The author wishes to acknowledge the insight of Alphonse Magnus, whose seminal study formed the inspiration for this work and who has freely offered many clear perceptions into the subject, the generous insights shared by Eric Rains, the kind interest of Mizan Rahman, the enthusiastic encouragement from Alexander Its, and the support of Peter Forrester.

\section{REFERENCES}

[1] R. Álvarez-Nodarse and J. C. Medem, q-classical polynomials and the $q$-Askey and Nikiforov-Uvarov tableaus, J. Comput. Appl. Math. 135 (2001), 197-223. MR 1850541. DOI 10.1016/S0377-0427(00)00585-9.

[2] D. Arinkin and A. Borodin, Moduli spaces of d-connections and difference Painlevé equations, Duke Math. J. 134 (2006), 515-556. MR 2254625. DOI 10.1215/S0012-7094-06-13433-6.

[3] - $\tau$-function of discrete isomonodromy transformations and probability, Compos. Math. 145 (2009), 747-772. MR 2507747. DOI 10.1112/S0010437X08003862.

[4] R. Askey and J. Wilson, Some Basic Hypergeometric Orthogonal Polynomials that Generalize Jacobi Polynomials, Mem. Amer. Math. Soc. 54, Amer. Math. Soc., Providence, 1985. MR 0783216. DOI 10.1090/memo/0319.

[5] N. M. Atakishiyev and S. K. Suslov, On the moments of classical and related polynomials, Rev. Mexicana Fís. 34 (1988), 147-151. MR 1034473.

[6] - "Difference hypergeometric functions" in Progress in Approximation Theory (Tampa, 1990), Springer Ser. Comput. Math. 19, Springer, New York, 1992, 1-31. MR 1240776. DOI 10.1007/978-1-4612-2966-7_1.

[7] G. Bangerezako, The factorization method for the Askey-Wilson polynomials, J. Comput. Appl. Math. 107 (1999), 219-232. MR 1701532. DOI 10.1016/S0377-0427(99)00090-4.

[8] - The fourth order difference equation for the Laguerre-Hahn polynomials orthogonal on special non-uniform lattices, Ramanujan J. 5 (2001), 167-181. MR 1857182. DOI 10.1023/A:1011487824004.

[9] G. Bangerezako and M. Foupouagnigni, Laguerre-Freud equations for the recurrence coefficients of the Laguerre-Hahn orthogonal polynomials on special non-uniform lattices, preprint, http://www.ictp.trieste.it (accessed 17 August 2015).

[10] G. Bangerezako and M. N. Hounkonnou, The factorization method for the general second-order q-difference equation and the Laguerre-Hahn polynomials on the general q-lattice, J. Phys. A 36 (2003), no. 3, 765-773. MR 1959430. DOI 10.1088/0305-4470/36/3/311.

[11] G. Bangerezako and A. P. Magnus, "The factorization method for the semi-classical polynomials" in Self-Similar Systems (Dubna, 1998), Joint Inst. Nuclear Res., Dubna, 1999, 295-300. MR 1819443. 
[12] R. J. Baxter, Exactly Solved Models in Statistical Mechanics, Academic Press, London, 1982. MR 0690578.

[13] S. Belmehdi and A. Ronveaux, Laguerre-Freud's equations for the recurrence coefficients of semi-classical orthogonal polynomials, J. Approx. Theory 76 (1994), 351368. MR 1272122. DOI 10.1006/jath.1994.1022.

[14] P. Biane, Orthogonal polynomials on the unit circle, q-gamma weights, and discrete Painlevé equations, Mosc. Math. J. 14 (2014), 1-27. MR 3221944.

[15] L. Boelen, C. Smet, and W. Van Assche, q-Discrete Painlevé equations for recurrence coefficients of modified q-Freud orthogonal polynomials, preprint, arXiv :0808.0982v1 [math.CA].

[16] A. Borodin, Discrete gap probabilities and discrete Painlevé equations, Duke Math. J. 117 (2003), 489-542. MR 1979052. DOI 10.1215/S0012-7094-03-11734-2.

[17] - Isomonodromy transformations of linear systems of difference equations, Ann. of Math. (2) 160 (2004), 1141-1182. MR 2144976. DOI 10.4007 /annals.2004.160.1141.

[18] A. Borodin and D. Boyarchenko, Distribution of the first particle in discrete orthogonal polynomial ensembles, Comm. Math. Phys. 234 (2003), 287-338. MR 1962463. DOI 10.1007/s00220-002-0767-3.

[19] Y. Chen and M. E. H. Ismail, Ladder operators and differential equations for orthogonal polynomials, J. Phys. A 30 (1997), no. 22, 7817-7829. MR 1616931. DOI 10.1088/0305-4470/30/22/020.

[20] - Ladder operators for q-orthogonal polynomials, J. Math. Anal. Appl. 345 (2008), 1-10. MR 2422628. DOI 10.1016/j.jmaa.2008.03.031.

[21] Y. Chen, M. E. H. Ismail, and W. Van Assche, Tau-function constructions of the recurrence coefficients of orthogonal polynomials, Adv. in Appl. Math. 20 (1998), 141-168. MR 1601371. DOI 10.1006/aama.1997.0574.

[22] R. S. Costas-Santos and F. Marcellán, q-Classical orthogonal polynomials: A general difference calculus approach, preprint, arXiv:math/0612097v5 [math.CA].

[23] A. S. Fokas, A. R. Its, and A. V. Kitaev, Discrete Painlevé equations and their appearance in quantum gravity, Comm. Math. Phys. 142 (1991), 313-344. MR 1137067.

[24] - The isomonodromy approach to matrix models in $2 D$ quantum gravity, Comm. Math. Phys. 147 (1992), 395-430. MR 1174420.

[25] P. J. Forrester, Log-Gases and Random Matrices, London Math. Soc. Monogr. Ser. 34, Princeton University Press, Princeton, 2010. MR 2641363. DOI $10.1515 / 9781400835416$.

[26] P. J. Forrester and N. S. Witte, Discrete Painlevé equations, orthogonal polynomials on the unit circle, and $N$-recurrences for averages over $U(N)-\mathrm{P}_{\mathrm{III}^{\prime}}$ and $\mathrm{P}_{\mathrm{V}} \tau$-functions, Int. Math. Res. Not. IMRN 2004, no. 4, 160-183. MR 2040326. DOI 10.1155/S1073792804131553.

[27] - Bi-orthogonal polynomials on the unit circle, regular semi-classical weights and integrable systems, Constr. Approx. 24 (2006), 201-237. MR 2239121. DOI 10.1007/s00365-005-0616-7.

[28] M. Foupouagnigni, On difference equations for orthogonal polynomials on nonuniform lattices, J. Difference Equ. Appl. 14 (2008), 127-174. MR 2383000. DOI 10.1080/10236190701536199.

[29] M. Foupouagnigni, M. N. Hounkonnou, and A. Ronveaux, "Laguerre-Freud equations for the recurrence coefficients of $D_{\omega}$ semi-classical orthogonal polynomials of class one" in Orthogonal Polynomials and Their Applications (Seville, 1997), J. Comput. Appl. Math. 99, 1998, 143-154. MR 1662690. DOI 10.1016/S0377-0427(98)00152-6. 
[30] G. Freud, Orthogonal Polynomials, Pergamon Press, Oxford, 1971.

[31] G. Gasper and M. Rahman, Basic Hypergeometric Series, 2nd ed., Encyclopedia Math. Appl. 96, Cambridge University Press, Cambridge, 2004. MR 2128719. DOI $10.1017 /$ CBO9780511526251.

[32] A. Ghressi and L. Khériji, The symmetrical $H_{q}$-semiclassical orthogonal polynomials of class one, SIGMA Symmetry Integrability Geom. Methods Appl. 5 (2009), Paper 076. MR 2529171. DOI 10.3842/SIGMA.2009.076.

[33] B. Grammaticos and A. Ramani, On a novel q-discrete analogue of the Painlevé VI equation, Phys. Lett. A 257 (1999), no. 5-6, 288-292. MR 1698774. DOI 10.1016/S0375-9601(99)00296-0.

[34] D. P. Gupta and D. R. Masson, "Solutions to the associated $q$-Askey-Wilson polynomial recurrence relation" in Approximation and Computation (West Lafayette, Ind., 1993), Internat. Ser. Numer. Math. 119, Birkhäuser, Boston, 1994, 273-284. MR 1333623.

[35] W. Hahn, Beiträge zur Theorie der Heineschen Reihen. Die 24 Integrale der Hypergeometrischen q-Differenzengleichung. Das q-Analogon der Laplace-Transformation, Math. Nachr. 2 (1949), 340-379. MR 0035344.

[36] — Über Orthogonalpolynome, die q-Differenzengleichungen genügen, Math. Nachr. 2 (1949), 4-34. MR 0030647.

[37] - Über die höheren Heineschen Reihen und eine einheitliche Theorie der sogenannten speziellen Funktionen, Math. Nachr. 3 (1950), 257-294. MR 0040557.

[38] - Über uneigentliche Lösungen linearer geometrischer Differenzengleichungen, Math. Ann. 125 (1952), 67-81. MR 0051426.

[39] A. Iatrou and J. A. G. Roberts, Integrable mappings of the plane preserving biquadratic invariant curves, II, Nonlinearity 15 (2002), 459-489. MR 1888861. DOI 10.1088/0951-7715/15/2/313.

[40] M. E. H. Ismail, "The Askey-Wilson operator and summation theorems" in Mathematical Analysis, Wavelets, and Signal Processing (Cairo, 1994), Contemp. Math. 190, Amer. Math. Soc., Providence, 1995, 171-178. MR 1354852. DOI $10.1090 /$ conm/190/02300.

[41] - "Functional equations and electrostatic models for orthogonal polynomials" in Random Matrix Models and Their Applications, Math. Sci. Res. Inst. Publ. 40, Cambridge University Press, Cambridge, 2001, 225-244. MR 1842788.

[42] - Difference equations and quantized discriminants for q-orthogonal polynomials, Adv. Appl. Math. 30 (2003), 562-589. MR 1973957. DOI 10.1016/S0196 -8858(02)00547-X.

[43] - Classical and Quantum Orthogonal Polynomials in One Variable, Encyclopedia Math. Appl. 98, Cambridge University Press, Cambridge, 2005. MR 2191786. DOI 10.1017/CBO9781107325982.

[44] M. E. H. Ismail, I. Nikolova, and P. Simeonov, Difference equations and discriminants for discrete orthogonal polynomials, Ramanujan J. 8 (2004), 475-502. MR 2130522. DOI 10.1007/s11139-005-0276-z.

[45] M. E. H. Ismail and M. Rahman, The associated Askey-Wilson polynomials, Trans. Amer. Math. Soc. 328 (1991), no. 1, 201-237. MR 1013333. DOI 10.2307/2001881.

[46] M. E. H. Ismail and P. Simeonov, q-Difference operators for orthogonal polynomials, J. Comput. Appl. Math. 233 (2009), 749-761. MR 2583013. DOI 10.1016/j.cam.2009.02.044. 
[47] M. E. H. Ismail and D. Stanton, q-Taylor theorems, polynomial expansions, and interpolation of entire functions, J. Approx. Theory 123 (2003), 125-146. MR 1985020. DOI 10.1016/S0021-9045(03)00076-5.

[48] M. E. H. Ismail and N. S. Witte, Discriminants and functional equations for polynomials orthogonal on the unit circle, J. Approx. Theory 110 (2001), 200-228. MR 1830537. DOI 10.1006/jath.2000.3540.

[49] A. R. Its, A. V. Kitaev, and A. S. Fokas, Matrix models of two-dimensional quantum gravity, and isomonodromic solutions of Painlevé "discrete equations" (in Russian), Zap. Nauchn. Sem. Leningrad. Otdel. Mat. Inst. Steklov. (LOMI) 187 (1991), 3-30; English translation in J. Math. Sci. 73 (1995), 415-429. MR 1111901. DOI $10.1007 / B F 02364564$.

[50] M. Jimbo and H. Sakai, A q-analog of the sixth Painlevé equation, Lett. Math. Phys. 38 (1996), 145-154. MR 1403067. DOI 10.1007/BF00398316.

[51] K. Kajiwara, T. Masuda, M. Noumi, Y. Ohta, and Y. Yamada, ${ }_{10} E_{9}$ solution to the elliptic Painlevé equation, J. Phys. A 36 (2003), no. 17, 263-272. MR 1984002. DOI 10.1088/0305-4470/36/17/102.

[52] - Hypergeometric solutions to the q-Painlevé equations, Int. Math. Res. Not. IMRN 2004, no. 47, 2497-2521. MR 2077840. DOI 10.1155/S1073792804140919.

[53] E. G. Kalnins and W. Miller, Jr., "Symmetry techniques for q-series: AskeyWilson polynomials" in Constructive Function Theory-86 Conference (Edmonton, 1986), Rocky Mountain J. Math. 19, 1989, 223-230. MR 1016175. DOI 10.1216/RMJ-1989-19-1-223.

[54] L. Khériji, An introduction to the $H_{q}$-semiclassical orthogonal polynomials, Methods Appl. Anal. 10 (2003), 387-411. MR 2059943.

[55] L. Khériji and P. Maroni, The $H_{q}$-classical orthogonal polynomials, Acta Appl. Math. 71 (2002), 49-115. MR 1893361. DOI 10.1023/A:1014597619994.

[56] R. Koekoek and R. F. Swarttouw, The Askey-scheme of hypergeometric orthogonal polynomials and its q-analogue, preprint, http://aw.twi.tudelft.nl/ koekoek /askey (accessed 17 August 2015).

[57] H. T. Koelink and T. H. Koornwinder, "q-special functions, a tutorial" in Deformation Theory and Quantum Groups with Applications to Mathematical Physics (Amherst, Mass., 1990), Contemp. Math. 134, Amer. Math. Soc., Providence, 1992, 141-142. MR 1187285. DOI 10.1090/conm/134/1187285.

[58] T. H. Koornwinder, The structure relation for Askey-Wilson polynomials, J. Comput. Appl. Math. 207 (2007), 214-226. MR 2345243. DOI 10.1016/j.cam.2006.10.015.

[59] P. A. Lesky, Eine Charakterisierung der klassischen kontinuierlichen-diskreten- und q-Orthogonal polynome, Shaker, Aachen, 2005.

[60] A. P. Magnus, "Associated Askey-Wilson polynomials as Laguerre-Hahn orthogonal polynomials" in Orthogonal Polynomials and Their Applications (Segovia, 1986), Lecture Notes in Math. 1329, Springer, Berlin, 1988, 261-278. MR 0973434. DOI $10.1007 / \mathrm{BFb} 0083366$.

[61] _ , "Painlevé-type differential equations for the recurrence coefficients of semiclassical orthogonal polynomials" in Orthogonal Polynomials and Their Applications (Evian-Les-Bains, 1992), J. Comput. Appl. Math. 57, 1995, 215-237. MR 1340938. DOI 10.1016/0377-0427(93)E0247-J. 
[62] — "Special nonuniform lattice (snul) orthogonal polynomials on discrete dense sets of points" in Orthogonality, Moment Problems and Continued Fractions (Delft, 1994), J. Comput. Appl. Math. 65, 1995, 253-265. MR 1379135. DOI 10.1016/0377-0427(95)00114-X.

[63] — Elliptic hypergeometric solutions to elliptic difference equations, SIGMA Symmetry Integrability Geom. Methods Appl. 5 (2009), Paper 038. MR 2506174. DOI 10.3842/SIGMA.2009.038.

[64] - Painlevé equations for semi-classical recurrence coefficients, preprint, arXiv: math/9409228v1 [math.CA].

[65] F. Marcellán and L. Salto, Discrete semi-classical orthogonal polynomials, J. Difference Equ. Appl. 4 (1998), 463-496. MR 1665164. DOI 10.1080/10236199808808156.

[66] P. Maroni, Une caractérisation des polynômes orthogonaux semi-classiques, C. R. Math. Acad. Sci. Paris Sér. I 301 (1985), 269-272. MR 0803215.

[67] P. Maroni and M. Mejri, The $I_{(q, \omega)}$ classical orthogonal polynomials, Appl. Numer. Math. 43 (2002), 423-458. MR 1941732. DOI 10.1016/S0168-9274(01)00180-5.

[68] - The symmetric $D_{\omega}$-semi-classical orthogonal polynomials of class one, Numer. Algorithms 49 (2008), 251-282. MR 2457103. DOI 10.1007/s11075 -008-9170-2.

[69] T. Masuda, Hypergeometric $\tau$-functions of the q-Painlevé system of type $E_{7}^{(1)}$, SIGMA Symmetry Integrability Geom. Methods Appl. 5 (2009), Paper 035. MR 2506177. DOI 10.3842/SIGMA.2009.035.

[70] M. Mejri, q-extension of some symmetrical and semi-classical orthogonal polynomials of class one, Appl. Anal. Discrete Math. 3 (2009), 78-87. MR 2499310. DOI 10.2298/AADM0901078M.

[71] M. Murata, H. Sakai, and J. Yoneda, Riccati solutions of discrete Painlevé equations with Weyl group symmetry of type $E_{8}^{(1)}$, J. Math. Phys. 44 (2003), 1396-1414. MR 1958273. DOI 10.1063/1.1531216.

[72] F. W. Nijhoff, On a q-deformation of the discrete Painlevé I equation and qorthogonal polynomials, Lett. Math. Phys. 30 (1994), 327-336. MR 1271093. DOI 10.1007/BF00751068.

[73] A. F. Nikiforov and S. K. Suslov, Systems of classical orthogonal polynomials of a discrete variable on nonuniform grids (in Russian), Akad. Nauk SSSR Inst. Prikl. Mat. Preprint 1985, no. 8. MR 0794000.

[74] - "Classical orthogonal polynomials of a discrete variable on nonuniform lattices" in Group Theoretical Methods in Physics, Vol. I (Yurmala, 1985), VNU Sci., Utrecht, 1986, 505-511. MR 0919767.

[75] - Classical orthogonal polynomials of a discrete variable on nonuniform lattices, Lett. Math. Phys. 11 (1986), 27-34. MR 0824673. DOI 10.1007/BF00417461.

[76] A. F. Nikiforov, S. K. Suslov, and V. B. Uvarov, Construction of particular solutions for a difference equation of hypergeometric type (in Russian), Akad. Nauk SSSR Inst. Prikl. Mat. Preprint 1984, no. 142. MR 0792163.

[77] - Classical orthogonal polynomials of a discrete variable on nonuniform grids (in Russian), Dokl. Akad. Nauk SSSR 291, no. 5 (1986), 1056-1059; English translation in Soviet. Math. Dokl. 34, no. 3 (1987), 576-579. MR 0872153.

[78] —, Classical Orthogonal Polynomials of a Discrete Variable (in Russian), Springer Ser. Comput. Phys., Springer, Berlin, 1991. MR 1149380. DOI 10.1007/978-3-642-74748-9. 
[79] C. M. Ormerod, N. S. Witte, and P. J. Forrester, Connection preserving deformations and q-semi-classical orthogonal polynomials, Nonlinearity 24 (2011), 24052434. MR 2819929. DOI 10.1088/0951-7715/24/9/002.

[80] P. I. Pastro, Orthogonal polynomials and some q-beta integrals of Ramanujan, J. Math. Anal. Appl. 112 (1985), 517-540. MR 0813618. DOI 10.1016/0022-247X(85)90261-6.

[81] M. Rahman, An integral representation of a $10 \varphi_{9}$ and continuous bi-orthogonal ${ }_{10} \varphi_{9}$ rational functions, Canad. J. Math. 38 (1986), 605-618. MR 0845667. DOI 10.4153/CJM-1986-030-6.

[82] - - -Wilson functions of the second kind, SIAM J. Math. Anal. 17 (1986), 1280-1286. MR 0853530. DOI 10.1137/0517089.

[83] M. Rahman and S. K. Suslov, Barnes and Ramanujan-type integrals on the q-linear lattice, SIAM J. Math. Anal. 25 (1994), 1002-1022. MR 1271323. DOI 10.1137/S0036141092233676.

[84] - The Pearson equation and the beta integrals, SIAM J. Math. Anal. 25 (1994), 646-693. MR 1266583. DOI 10.1137/S003614109222874X.

[85] E. M. Rains, An isomonodromy interpretation of the hypergeometric solution of the elliptic Painlevé equation, SIGMA Symmetry Integrability Geom. Methods Appl. 7 (2011), Paper 088. MR 2861188.

[86] H. Sakai, Rational surfaces associated with affine root systems and geometry of the Painlevé equations, Comm. Math. Phys. 220 (2001), 165-229. MR 1882403. DOI 10.1007/s002200100446.

[87] - A q-analog of the Garnier system, Funkcial. Ekvac. 48 (2005), 273-297. MR 2177121. DOI 10.1619/fesi.48.273.

[88] - Lax form of the q-Painlevé equation associated with the $A_{2}^{(1)}$ surface, J. Phys. A 39 (2006), no. 39, 12203-12210. MR 2266221. DOI 10.1088/0305-4470/39/39/S13.

[89] V. P. Spiridonov and A. S. Zhedanov, "Generalized eigenvalue problem and a new family of rational functions biorthogonal on elliptic grids" in Special Functions 2000: Current Perspective and Future Directions (Tempe, 2000), NATO Sci. Ser. II Math. Phys. Chem. 30, Kluwer Acad., Dordrecht, 2001, 365-388. MR 2006295. DOI 10.1007/978-94-010-0818-1_14.

[90] - Elliptic grids, rational functions, and the Padé interpolation, Ramanujan J. 13 (2007), 285-310. MR 2281167. DOI 10.1007/s11139-006-0253-1.

[91] S. K. Suslov, On the theory of difference analogues of special functions of hypergeometric type, Uspekhi Mat. Nauk 44 (1989), 185-226; English translation in Russian Math. Surveys 44 (1989), 227-278. MR 0998364. DOI 10.1070/RM1989v044n02ABEH002045.

[92] G. Szegő, Orthogonal Polynomials, 3rd ed., Colloq. Publ. 23, Amer. Math. Soc., Providence, 1967. MR 0310533.

[93] W. Van Assche, "Discrete Painlevé equations for recurrence coefficients of orthogonal polynomials" in Difference Equations, Special Functions and Orthogonal Polynomials, World Sci. Publ., Hackensack, N.J., 2007, 687-725. MR 2451211. DOI 10.1142/9789812770752_0058.

[94] N. S. Witte, Bi-orthogonal systems on the unit circle, regular semi-classical weights and integrable systems, II, J. Approx. Theory 161 (2009), 565-616. MR 2563070. DOI 10.1016/j.jat.2008.11.017. 
[95] - Biorthogonal systems on the unit circle, regular semiclassical weights, and the discrete Garnier equations, Int. Math. Res. Not. IMRN 2009, no. 6, 988-1025. MR 2487490. DOI 10.1093/imrn/rnn152.

[96] — Deformations of the Askey-Wilson polynomials and their Bäcklund transformations, preprint, 2010.

[97] - On a family of integrals that extend the Askey-Wilson integral, J. Math. Anal. App. 421 (2015), 1101-1130. MR 3258309.

[98] Y. Yamada, A Lax formalism for the elliptic difference Painlevé equation, SIGMA Symmetry Integrability Geom. Methods Appl. 5 (2009), Paper 042. MR 2506170. DOI 10.3842/SIGMA.2009.042.

[99] - Lax formalism for q-Painlevé equations with affine Weyl group symmetry of type $E_{n}^{(1)}$, Int. Math. Res. Not. IMRN 2011, no. 17, 3823-3838. MR 2836394.

School of Mathematics and Statistics

University of Melbourne

Victoria 3010

Australia

n.witte@ms . unimelb.edu.au 